BNL-113453-2017-JA

\title{
White paper on nuclear astrophysics and low-energy nuclear physics Part 2: Low-energy nuclear physics
}

Mark A. Riley, Charlotte Elster, Joe Carlson, Michael P. Carpenter, Richard Casten, Paul Fallon, Alexandra Gade, Carl Gross, Gaute Hagen, Anna C. Hayes, Douglas W. Higinbotham, Calvin R. Howell, Charles J. Horowitz, Kate L. Jones, Filip G. Kondev, Suzanne Lapi, Augusto Macchiavelli, Elizabeth A. McCutchen, Joe Natowitz, Witold Nazarewicz, Thomas Papenbrock, Sanjay Reddy, Martin J. Savage, Guy Savard, Bradley M. Sherrill, Lee G. Sobotka, Mark A. Stoyer, M. Betty Tsang, Kai Vetter, Ingo Wiedenhoever, Alan H. Wuosmaa, Sherry Yennello

Submitted to Progress in Particle and Nuclear Physics

January 13, 2017

National Nuclear Data Center

Brookhaven National Laboratory

U.S. Department of Energy
USDOE Office of Science (SC),
Nuclear Physics (NP) (SC-26)

Notice: This manuscript has been authored by employees of Brookhaven Science Associates, LLC under Contract No.DE-SC0012704 with the U.S. Department of Energy. The publisher by accepting the manuscript for publication acknowledges that the United States Government retains a non-exclusive, paid-up, irrevocable, world-wide license to publish or reproduce the published form of this manuscript, or allow others to do so, for United States Government purposes. 


\section{DISCLAIMER}

This report was prepared as an account of work sponsored by an agency of the United States Government. Neither the United States Government nor any agency thereof, nor any of their employees, nor any of their contractors, subcontractors, or their employees, makes any warranty, express or implied, or assumes any legal liability or responsibility for the accuracy, completeness, or any third party's use or the results of such use of any information, apparatus, product, or process disclosed, or represents that its use would not infringe privately owned rights. Reference herein to any specific commercial product, process, or service by trade name, trademark, manufacturer, or otherwise, does not necessarily constitute or imply its endorsement, recommendation, or favoring by the United States Government or any agency thereof or its contractors or subcontractors. The views and opinions of authors expressed herein do not necessarily state or reflect those of the United States Government or any agency thereof. 


\section{Accepted Manuscript}

White paper on nuclear astrophysics and low-energy nuclear physics Part 2: Low-energy nuclear physics

Mark A. Riley, Charlotte Elster, Joe Carlson, Michael P. Carpenter, Richard Casten, Paul Fallon, Alexandra Gade, Carl Gross, Gaute Hagen, Anna C. Hayes, Douglas W. Higinbotham, Calvin R. Howell, Charles J. Horowitz, Kate L. Jones, Filip G. Kondev, Suzanne Lapi, Augusto Macchiavelli, Elizabeth A. McCutchen, Joe Natowitz, Witold Nazarewicz, Thomas Papenbrock, Sanjay Reddy, Martin J. Savage, Guy Savard, Bradley M. Sherrill, Lee G. Sobotka, Mark A. Stoyer, M. Betty Tsang, Kai Vetter, Ingo Wiedenhoever, Alan H. Wuosmaa, Sherry Yennello

PII: S0146-6410(16)30072-2

DOI: $\quad$ http://dx.doi.org/10.1016/j.ppnp.2016.11.002

Reference: $\quad$ JPPNP 3625

Please cite this article as: M.A. Riley, C. Elster, J. Carlson, M.P. Carpenter, R. Casten, P. Fallon, A. Gade, C. Gross, G. Hagen, A.C. Hayes, D.W. Higinbotham, C.R. Howell, C.J. Horowitz, K.L. Jones, F.G. Kondev, S. Lapi, A. Macchiavelli, E.A. McCutchen, J. Natowitz, W. Nazarewicz, T. Papenbrock, S. Reddy, M.J. Savage, G. Savard, B.M. Sherrill, L.G. Sobotka, M.A. Stoyer, M. Betty Tsang, K. Vetter, I. Wiedenhoever, A.H. Wuosmaa, S. Yennello, White paper on nuclear astrophysics and low-energy nuclear physics Part 2: Low-energy nuclear physics, Progress in Particle and Nuclear Physics (2017), http://dx.doi.org/10.1016/j.ppnp.2016.11.002

This is a PDF file of an unedited manuscript that has been accepted for publication. As a service to our customers we are providing this early version of the manuscript. The manuscript will undergo copyediting, typesetting, and review of the resulting proof before it is published in its final form. Please note that during the production process errors may be discovered which could affect the content, and all legal disclaimers that apply to the journal pertain. 


\section{White Paper on Nuclear Astrophysics and Low-Energy Nuclear Physics}

\section{Part 2: Low-Energy Nuclear Physics}

January 31, 2015 


\title{
Author Listing for LENP White Paper (submitted by WP Convener Mark. A. Riley) :
}

\author{
Mark A. Riley \\ Department of Physics, Florida State University, Tallahassee, Florida 32306, USA \\ Charlotte Elster \\ Institute of Nuclear and Particle Physics, and Department of Physics and Astronomy, Ohio \\ University, Athens, Ohio 45701, USA
}

And

Joe Carlson Los Alamos National Laboratory

Theoretical Division, Los Alamos National Laboratory, Los Alamos, New Mexico 87545, USA

Michael P. Carpenter

Physics Division, Argonne National Laboratory, Argonne, Illinois 60439, USA

Richard Casten

Wright Nuclear Structure Laboratory, Yale University, New Haven, Connecticut 06520, USA

Paul Fallon

Nuclear Science Division, Lawrence Berkeley National Laboratory, Berkeley, California 94720, USA

\author{
Alexandra Gade \\ National Superconducting Cyclotron Laboratory, Michigan State University, East Lansing, \\ Michigan 48824, USA \\ Department of Physics and Astronomy, Michigan State University, East Lansing, Michigan \\ 48824, USA \\ Carl Gross \\ Physics Division, Oak Ridge National Laboratory, Oak Ridge, Tennessee 37931, USA \\ Gaute Hagen \\ Physics Division, Oak Ridge National Laboratory, Oak Ridge, TN 37831, USA \\ Department of Physics and Astronomy, University of Tennessee, Knoxville, TN 37996, USA
}

Anna C. Hayes

Los Alamos National Laboratory, Los Alamos, New Mexico 87544, USA

Douglas W. Higinbotham

Jefferson Lab, Newport News, Virginia 23606, USA

Calvin R. Howell

Department of Physics, Duke University, Durham, North Carolina 27708, USA

Triangle Universities Nuclear Laboratory, Durham, North Carolina 27708, USA

Charles J. Horowitz

Physics Department and Nuclear Theory Center, Indiana University, Bloomington, Indiana 47405, USA 
Kate L. Jones

Department of Physics and Astronomy, University of Tennessee, Knoxville, Tennessee 37996, USA

Filip G. Kondev

Nuclear Engineering Division, Argonne National Laboratory, Argonne, IL 60439, USA

Suzanne Lapi

Department of Chemistry, Washington University in St. Louis, One Brookings Drive, St. Louis, Missouri 63139, USA

Augusto Macchiavelli

Nuclear Science Division, Lawrence Berkeley National Laboratory, Berkeley, California 94720, USA

Elizabeth A. McCutchen

Brookhaven National Laboratory P.O. Box 5000 Upton, NY 11973, USA

Joe Natowitz

Cyclotron Institute, Texas A\&M University, College Station, Texas 77843, USA

Witold Nazarewicz

Department of Physics and Astronomy and FRIB Laboratory, Michigan State University, East Lansing, Michigan 48824, USA

Institute of Theoretical Physics, Faculty of Physics, University of Warsaw, 02-093 Warsaw,

Poland

Thomas Papenbrock

Department of Physics and Astronomy, University of Tennessee, Knoxville, Tennessee 37996, USA

Physics Division, Oak Ridge National Laboratory, Oak Ridge, Tennessee 37831, USA

Sanjay Reddy

Institute for Nuclear Theory, University of Washington, Seattle, Washington, USA

Department of Physics, University of Washington, Seattle, Washington, USA

Martin J. Savage

Institute for Nuclear Theory, University of Washington, Seattle, Washington 98195-1550, USA

Guy Savard

Physics Division, Argonne National Laboratory, Argonne, Illinois 60439, USA

Bradley M. Sherrill

National Superconducting Cyclotron Laboratory, Michigan State University, East Lansing, Michigan 48824, USA

Department of Physics and Astronomy, Michigan State University, East Lansing, Michigan 48824, USA

Lee G. Sobotka

Departments of Chemistry and Physics, Washington University, St. Louis, Missouri 63130, USA 
Mark A. Stoyer

Lawrence Livermore National Laboratory, Livermore, California 94551, USA

M. Betty Tsang

National Superconducting Cyclotron Laboratory, Michigan State University, East Lansing, Michigan 48824

Kai Vetter

Applied Physics Program, Nuclear Science Division, LBNL, Berkeley, CA 94720

Ingo Wiedenhoever

Department of Physics, Florida State University, Tallahassee, Florida 32306, USA

Alan H. Wuosmaa

Department of Physics, University of Connecticut, Storrs, Connecticut 06268-3046, USA

Sherry Yennello

Cyclotron Institute and Department of Chemistry, Texas A\&M University, College Station, Texas 77843, USA 
Table of Contents

LENP Town Meeting Organizing Committee and LENP White Paper Writing Group ...........3

1. Joint Executive Summary from the Nuclear Astrophysics and Low-Energy Nuclear

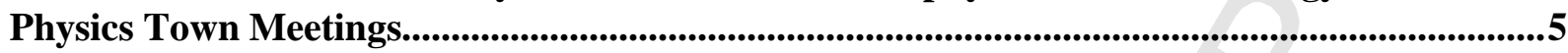

2. Executive Summary of the Low-Energy Nuclear Physics Town Meeting ...............................9

3. White Paper: “Low-Energy Nuclear Physics Town Meeting”.................................................14

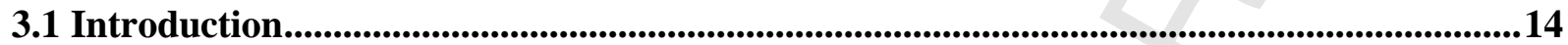

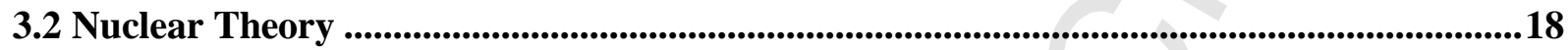

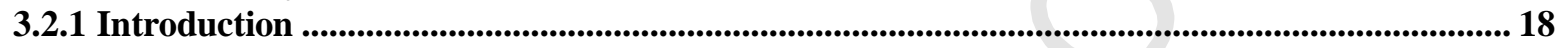

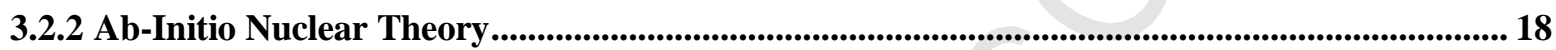

3.2.3 Nuclear Reaction Theory ......................................................................................................................... 20

3.2.4 Density Functional and Other Theories for Heavy Nuclei ................................................................. 22

3.2.5 Nuclear Theory and Nuclear Astrophysics.............................................................................................. 24

3.2.6 Connections to All of Nuclear Science and Beyond .............................................................................. 26

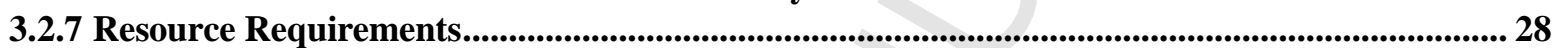

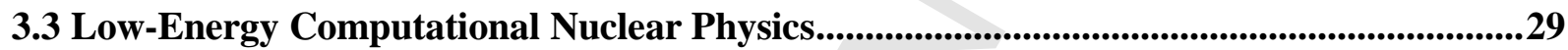

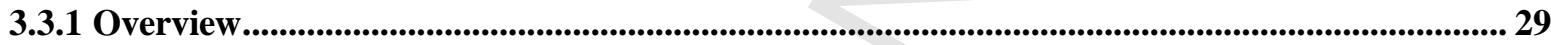

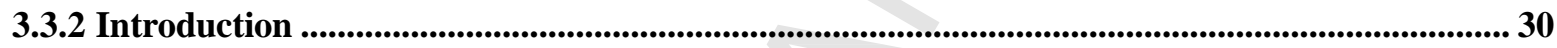

3.3.3 Near-Term and Long-Term Scientific Opportunities.............................................................................. 30

3.3.4 A Selection of Focused Objectives ........................................................................................................... 31

3.3.5 Major Accomplishments Since the Last Long Range Plan ............................................................. 33

3.3.6 Near-Term and Long-Term Resource Needs ............................................................................. 34

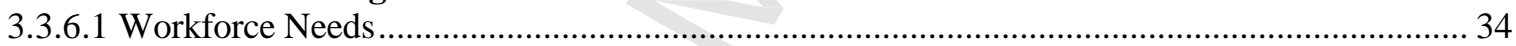

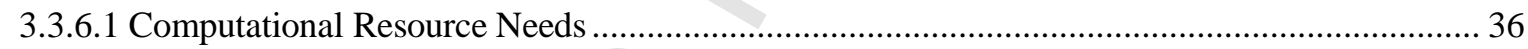

3.3.7 Scientific Impact on Other Fields and Interdisciplinary Connections ........................................ 38

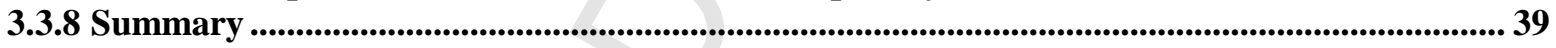

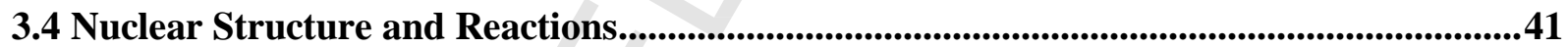

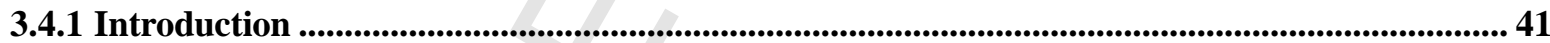

3.4.2 Progress Since the Last Long Range Plan .............................................................................................. 41

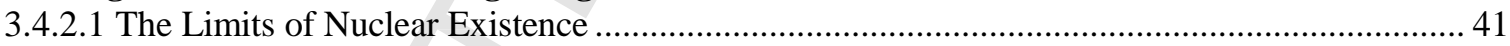

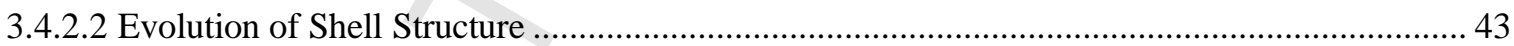

3.4.2.3 Benchmarking Developments in Nuclear Structure Theory ........................................................ 44

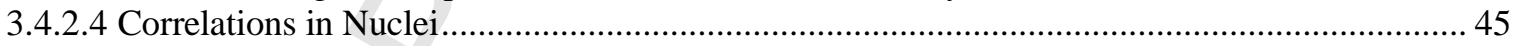

3.4.2.5 Symmetries and Simplicities in Complex Systems .......................................................................46

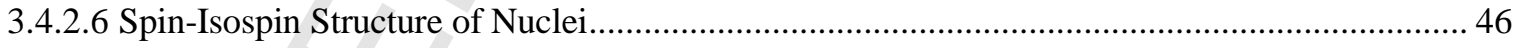

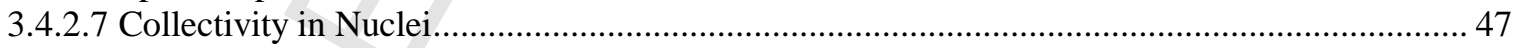

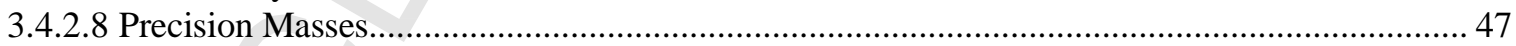

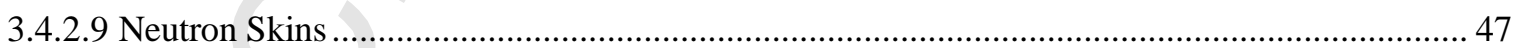

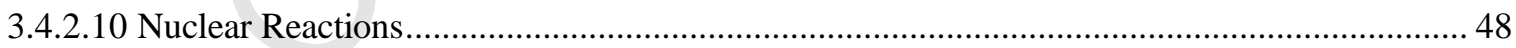

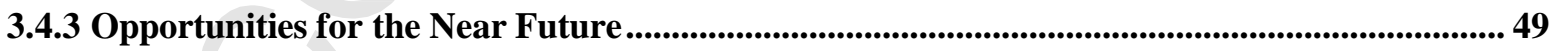

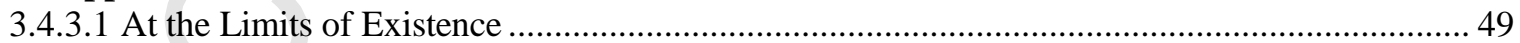

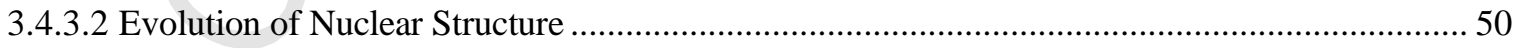

3.4.3.3 Collective Nuclear Degrees of Freedom and Simple Patterns in Complex Nuclei..................... 50

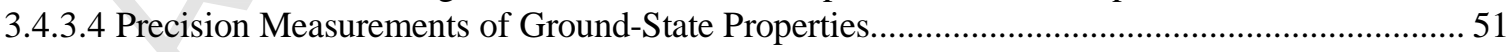

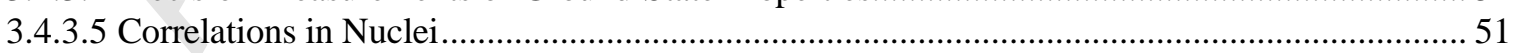

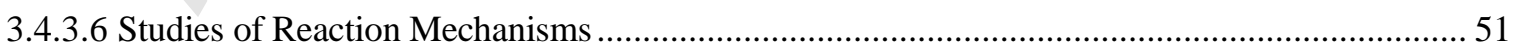

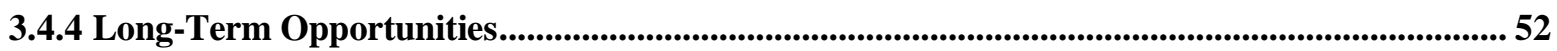


3.4.5 At the Intersection to Hadronic Physics - Near-Term and Long-Term Opportunities for Nuclear Structure Research at Jefferson Lab .............................................................................................. 53

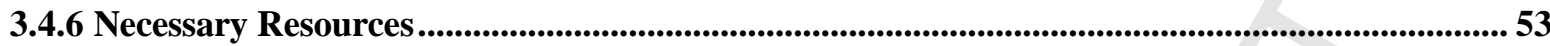

3.4.7 Scientific Impact on Other Fields and Interdisciplinary Connections ........................................ 55

3.5 Nature of Dilute and Dense Nuclear Matter and Equation of State ......................................57

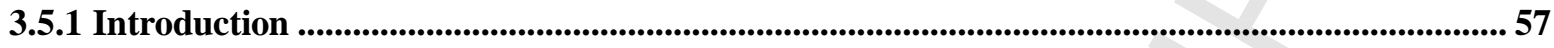

3.5.2 Compelling Scientific Opportunities: Near-Term and Long-Term.............................................. 57

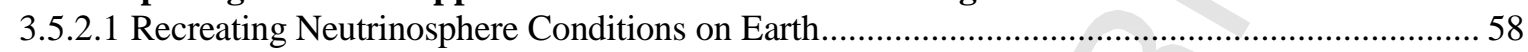

3.5.2.2. Impact of Clustering at Very Low Density and in Nuclear Surfaces ......................................... 59

3.5.2.3. Astrophysical Relevant Fission and Fusion Measurements ..................................................... 59

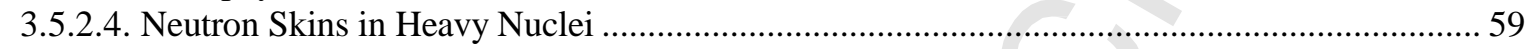

3.5.2.5 Density Dependence of Symmetry Energy Around Saturation Density .........................................6 60

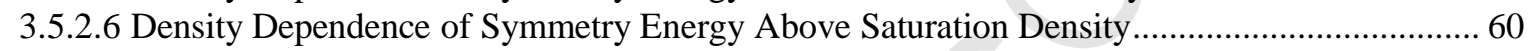

3.5.3 Major Accomplishments Since the Last Long Range Plan .......................................................6 60

3.5.4 Near-Term and Long-Term Resources Needed .................................................................................... 63

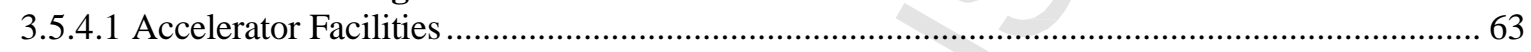

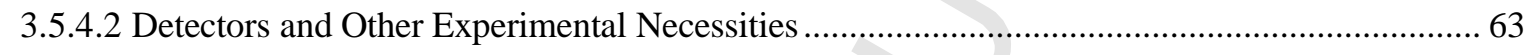

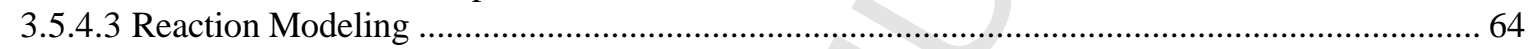

3.5.5 Scientific Impact on Other Fields and Interdisciplinary Connections .......................................... 65

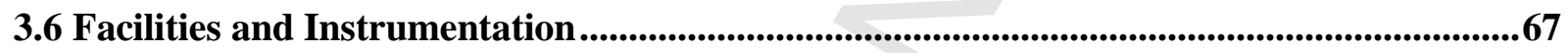

3.6.1 Infrastructure and Resources in Nuclear Structure and Nuclear Astrophysics ........................... 67

National Laboratory and University Accelerator Facilities .......................................................................67

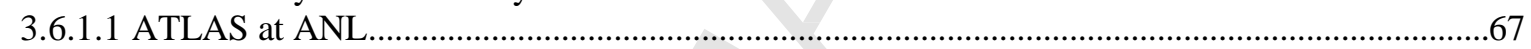

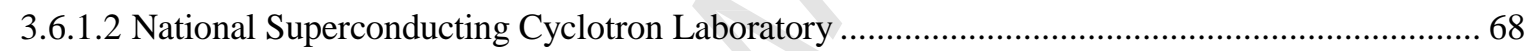

3.6.1.3 Low-Energy University Based Facilities (ARUNA).........................................................................69

3.6.1.4 Thomas Jefferson National Accelerator Facility................................................................................ 71

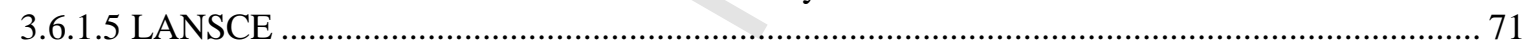

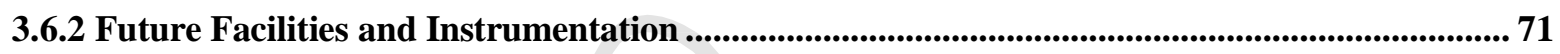

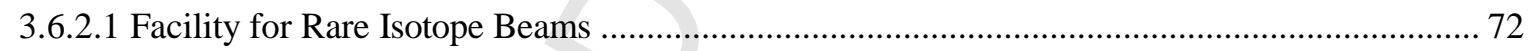

3.6.2.2 Equipment Development for Low-Energy Nuclear Physics ....................................................... 72

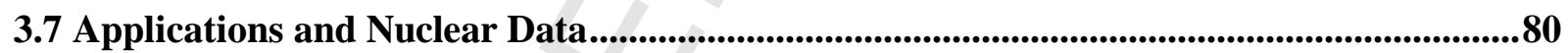

3.7.1 Accomplishments Since the Last Long Range Plan................................................................. 80

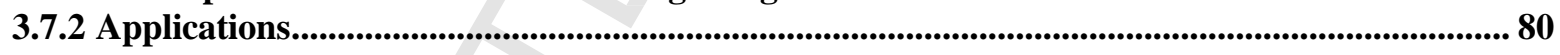

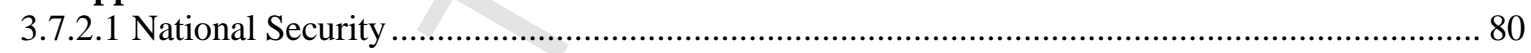

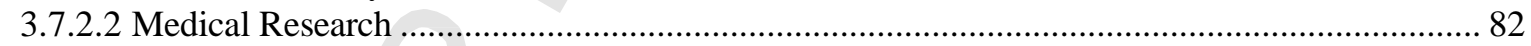

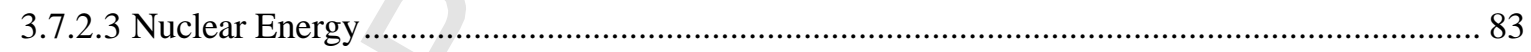

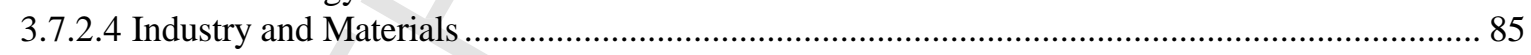

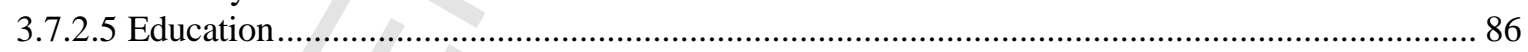

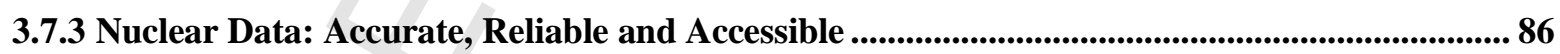

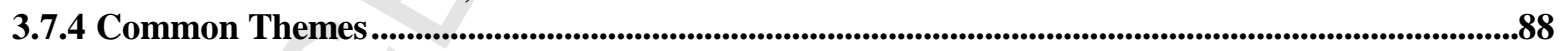

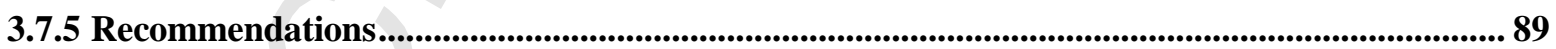

Appendix: Nuclear Astrophysics and Low-Energy Nuclear Physics Town Meetings Joint

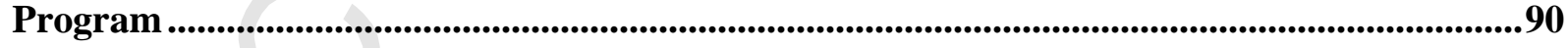




\section{LENP Town Meeting Organizing Committee and LENP White Paper Writing Group}

\section{Conveners}

Charlotte Elster

Ohio University

Mark Riley

Florida State University

Note: The Low-Energy Nuclear Physics Town Meeting was organized in association with the Nuclear Astrophysics Town Meeting whose conveners were Hendrik Schatz (Michigan State University) and Michael Wiescher (University of Notre Dame).

\section{Local Organizer}

Sherry Yennello Texas A\&M University

Town Meetings Joint Resolution Session Chair

Richard Casten Yale University

Working Groups and Organizers

Theory for Low-Energy Nuclear Science

Joe Carlson (Chair) Los Alamos National Laboratory

Gaute Hagen

Oak Ridge National Laboratory

Charlotte Elster

Ohio University

Witold Nazarewicz

Michigan State University

Thomas Papenbrock

University of Tennessee, Knoxville

Sanjay Reddy

University of Washington

Future Computational Needs in Nuclear Physics

Joe Carlson

Los Alamos National Laboratory

Martin Savage (Chair) University of Washington

\section{Nuclear Structure and Reactions}

Michael Carpenter

Paul Fallon

Alexandra Gade (Chair)

Douglas Higinbotham

Kate Jones

Alan Wuosmaa

\author{
Argonne National Lab \\ Lawrence Berkeley National Laboratory \\ Michigan State University \\ Thomas Jefferson National Laboratory \\ University of Tennessee, Knoxville \\ University of Connecticut
}


Nature of Dilute and Dense Nuclear Matter and the Equation of State

Charles Horowitz Indiana University

Joe Natowitz Texas A\&M University

Lee Sobotka Washington University

Betty Tsang (Chair) Michigan State University

\section{Facilities and Instrumentation}

Carl Gross

Oak Ridge National Laboratory

Calvin Howell

Duke University

Augusto Macchiavelli

Lawrence Berkeley National Laboratory

Guy Savard (Chair)

Argonne National Laboratory

Bradley Sherrill

Michigan State University

Ingo Wiedenhoever

Florida State University

\section{Applications and Nuclear Data}

Mark Stoyer (Chair)

Lawrence Livermore National Laboratory

Anna Hayes

Los Alamos National Laboratory

Suzanne Lapi

Washington University

Kai Vetter

University of California at Berkeley

Filip Kondev

Argonne National Laboratory

Elizabeth McCutchen

Brookhaven National Laboratory 


\section{Joint Executive Summary from the Nuclear Astrophysics and Low-Energy Nuclear Physics Town Meetings}

In preparation for the 2015 NSAC Long Range Plan (LRP), the DNP town meetings on Nuclear Astrophysics and Low-Energy Nuclear Physics were held at the Mitchell Center on the campus of Texas A\&M University August 21-23, 2014. Participants met in a number of topic-oriented working groups to discuss progress since the 2007 LRP, compelling science opportunities, and the resources needed to advance them. These considerations were used to determine priorities for the next five to ten years. Approximately 270 participants attended the meetings, coming from US national laboratories, a wide range of US universities and other research institutions and universities abroad.

The low-energy nuclear physics and the nuclear astrophysics communities unanimously endorsed a set of joint resolutions. The full text of the resolutions adopted at the Town Meetings is included at the end of this document. The joint resolutions were condensed from the individual recommendations of the two town meetings in order to recognize the highest priorities of the two fields. The resulting joint resolutions reflect hard choices, as not all priority items from the individual meetings are included. The communities strongly hope that the broader needs can be realized in time.

The resolutions represent a plan to address the compelling scientific opportunities in the study of atomic nuclei and their role in the cosmos. The intellectual challenges of our subfields were captured well in overarching questions from the 2012 NRC Decadal Survey of Nuclear Physics:

- How did visible matter come into being and how does it evolve?

- How does subatomic matter organize itself and what phenomena emerge?

- Are the fundamental interactions that are basic to the structure of matter fully understood?

- How can the knowledge and technological progress provided by nuclear science best be used to benefit society?

Answers to these questions require a deeper understanding of atomic nuclei, both theoretically and experimentally, than we have now. The last decade already saw considerable progress in understanding of the nucleus and its role in the universe. New ideas, combined with new experimental techniques, major leaps in computing power and impressive improvements in experimental capabilities resulted in discoveries leading to quantitative and qualitative changes in our understanding of nuclear and astrophysical phenomena.

A comprehensive discussion of the broad scientific opportunities and challenges will be provided in the individual town meeting reports. Low-Energy nuclear physics research addresses the existence of atomic nuclei, their limits, and their underlying nature. It also aims to describe interactions between nuclei and dynamical processes such as fission. The ultimate goal is to develop a predictive understanding of nuclei and their interactions grounded in fundamental QCD and electroweak theory. Nuclear astrophysics addresses important scientific questions at the intersection between nuclear physics and astrophysics: the chemical history of the Universe, the evolution of stars, stellar explosions, the nature of dense matter, and the ultimate fate of visible matter.

Both fields are poised for breakthroughs. New astronomical data, such as high resolution abundance distributions from very early stars; the neutrino fluxes from our sun, nearby supernovae, or possibly even the Big Bang; and anticipated gravity waves detected from merging neutron stars, will only be interpreted successfully if the relevant nuclear processes are understood. Exploration of elements and isotopes at the extremes of $\mathrm{N}$ and $\mathrm{Z}$ will provide the insights needed for a comprehensive understanding of nuclei. This exploration may reveal novel quantum many-body features and lead us toward a deeper understanding of 
complex quantum systems and of the mechanisms responsible for the emergent features found in atomic nuclei.

More broadly, science and society rely on our understanding of the atomic nucleus. Its relevance spans the dimensions of distance from $10^{-15} \mathrm{~m}$ (the proton radius) to $12 \mathrm{~km}$ (neutron star radius) and the evolutionary history of the universe from fractions of a second after the Big Bang to today; i.e., 13.8 billion years later. As reaffirmed by the 2012 National Academies of Sciences' decadal study "Nuclear Physics: Exploring the Heart of Matter," the path to understanding the nucleus requires the completion of the Facility for Rare Isotope Beams (FRIB) and its effective operation. Unprecedented access to a vast new terrain of nuclei will result in scientific breakthroughs and major advances in our understanding of nuclei and their role in the cosmos, and will open new avenues in cross-discipline contributions in basic sciences, national security, and other societal applications.

Based on this the first joint resolution was: 1. The highest priority in low-energy nuclear physics and nuclear astrophysics is the timely completion of the Facility for Rare Isotope Beams and the initiation of its full science program.

While FRIB is the top priority of both subfields, there are other capabilities needed to reach the scientific goals of the field. In arriving at a joint set of resolutions, the Town Meeting participants addressed priorities for the field as a whole. What emerged is a coherent plan that pursues key scientific opportunities by leveraging existing and future facilities. The plan involves continuation of forefront research activities, development of needed theory, and initiation of a focused set of new equipment initiatives. While many specific ideas were discussed, it was decided to approve wording that made clear that the community is asking for the base set of needs while recognizing that some initiatives may have to be delayed. The consensus minimum need is outlined in the following.

The science goals of Low-Energy Nuclear Physics and Nuclear Astrophysics require studies with probes ranging from stable and radioactive nuclei, to photons, neutrons, and electrons. While FRIB will explore uncharted regions of the nuclear chart and produce rare isotopes important for study of explosive environments, there is a key component of the program that links this exploration to studies of near-stable isotopes. The ATLAS stable beam facility has world-unique capabilities that will enable necessary precision studies near stability and at the limits of atomic number. Many aspects of stellar evolution, in particular the long quiescent phases of stellar evolution defining the conditions and providing the seed for subsequent cataclysmic developments, are driven by reactions of stable isotopes that will be studied at stable-beam accelerators. The electron beam at JLab provides a unique capability for probing the shortrange part of the nuclear force in nuclei and the modification of nucleons in the nuclear medium. The photon beam at HI $\gamma \mathrm{S}$ and the neutron beams at Los Alamos provide unique, important information. The university accelerator labs have a special role. They contribute cutting edge science, targeted research programs of longer duration, critical developments of techniques and equipment, combined with hands-on education.

For success in the long term, including success of FRIB, we must adequately operate current low-energy facilities and support the research groups that utilize these facilities to advance science and to foster an experienced and engaged research community. Adequate operations require that planned upgrades, already in baseline budgets, proceed, and current levels of research activities be enhanced. Significant facility reductions have occurred since 2007, including the closure of HRIBF and WNSL. These closures, coupled with limited availability of research hours at facilities worldwide, has resulted in a loss of research opportunities that can be restored in part by the development of a multi-user capability at ATLAS. 
The Town Meetings recognized that the NSF has played a leading role in the establishment and operation of one of the nation's flagship nuclear science facilities, NSCL, as well as in supporting university laboratories that carry out forefront nuclear physics and nuclear astrophysics research. There is the opportunity for continued leadership by support of cutting edge nuclear physics and transformative research extending well into the era of FRIB operations. This LRP can make the case for continued strong nuclear physics funding by NSF. Continued NSF leadership can be accomplished by an exciting plan that includes the effective operations at NSCL and NSF university laboratories, proposed upgrades to ReA3 at NSCL, and support for nuclear astrophysics initiatives such as an underground accelerator.

Hence, the Town Meeting participants recommended: 2a. We recommend appropriate support for operations and planned upgrades at ATLAS, NSCL, and university-based laboratories, as well as for the utilization of these and other facilities, for continued scientific leadership. Strong support for research groups is essential.

Significant progress in the theory of nuclei and the astrophysical environments in which they are embedded is a key component of the scientific goals of the field. A strong theory effort needs to go hand in hand with future experimental programs. The proposed FRIB Theory Center, a modest-scale national effort comprising a broad theory community, will therefore be important for the success of FRIB. (A brief overview of this effort will be summarized in a separate document.) The FRIB Theory Center will complement the NSF funded Joint Institute for Nuclear Astrophysics that facilitates the communications within the nuclear astrophysics community. JINA's efforts are essential for the interpretation of nuclear physics experiments and stellar observations in the framework of new theoretical developments. Its continuous operation is a main goal for the nuclear astrophysics community. This need for broad theory support resulted in part two of the second recommendation: $\mathbf{2 b}$. We recommend enhanced support for theory in low-energy nuclear science and nuclear astrophysics, which is critical to realize the full scientific promise of our fields.

In addition to the needs articulated above, a targeted set of new instrumentation and accelerator investments are necessary. FRIB will be a world-leading accelerator and will yield the best science when coupled with world-leading equipment. The community has identified and prioritized key detector systems and re-accelerator upgrades that will enable effective utilization of FRIB with the highest science potential. In the 2007 LRP the major new detector endorsed was GRETA and, at that time, it was recognized that an astrophysics separator would be needed (which is now named SECAR). These stateof-the-art systems are still top priority and highly anticipated by the community. While existing equipment can and will be used at FRIB, major advances in other detector and separator technology continue to take place. To this end, the community has developed exciting ideas for critical new equipment. Not all can be realized immediately, but a targeted suite to address the highest-priority research programs is needed.

In the area of accelerator investments, there is an opportunity for NSF to play a leading role by upgrading ReA3 and by establishing an underground accelerator for nuclear astrophysics. The community has long advocated for these developments.

In order to prepare the future and advance the science goals of the community, the third part of the second resolution states: 2c. We recommend targeted major instrumentation and accelerator investments to realize the discovery potential of our fields.

Two additional joint resolutions were adopted to support the main conclusions of the Computational Town Meeting and the Education and Innovation Town Meeting. 


\section{Full text of the Joint Resolutions:}

Science and society rely on our understanding of the atomic nucleus. Its relevance spans the dimensions of distance from $10^{-15} \mathrm{~m}$ (the proton's radius) to $12 \mathrm{~km}$ (the neutron star radius) and timescales from fractions of a second after the Big Bang to today, i.e. 13.8 billion years later. As reaffirmed by the 2012 National Academies of Sciences' decadal study "Nuclear Physics: Exploring the Heart of Matter", the path to understanding the nucleus requires the completion of the Facility for Rare Isotope Beams (FRIB) and its effective operation. Unprecedented access to a vast new terrain of nuclei will result in scientific breakthroughs and major advances in our understanding of nuclei and their role in the cosmos, and will open new avenues in cross-discipline contributions in basic sciences, national security, and other societal applications.

- The highest priority in low-energy nuclear physics and nuclear astrophysics is the timely completion of the Facility for Rare Isotope Beams and the initiation of its full science program.

In support of our science goals we must continue forefront research, exploiting existing facilities, develop new capabilities and equipment, and enable major advances in nuclear theory.

- We recommend appropriate support for operations and planned upgrades at ATLAS, NSCL, and university-based laboratories, as well as for the utilization of these and other facilities, for continued scientific leadership. Strong support for research groups is essential.

- We recommend enhanced support for theory in low-energy nuclear science and nuclear astrophysics, which is critical to realize the full scientific promise of our fields.

- We recommend targeted major instrumentation and accelerator investments to realize the discovery potential of our fields.

Realizing the scientific potential of Low-Energy Nuclear Science and Nuclear Astrophysics demands large-scale computations in nuclear theory that exploit the US leadership in high-performance computing.

- We endorse the recommendations of the 2014 Computational Nuclear Physics Meeting: "Capitalizing on the pre-exascale systems of 2017 and beyond requires significant new investments in people, advanced software, and complementary capacity computing directed toward nuclear theory."

Education, outreach, and innovation are key components of any vision of the future of the field of nuclear science. Our fields play a leading role in education and training of the nation's nuclear science workforce. They are ideally positioned to advance applications in medicine, energy, national security, and material science. The health of this field is required to train the talented national workforce needed to assure continuing societal benefits in these critical areas. Continuation of this role is a major goal of our fields.

- We endorse the recommendation of the DNP Town Meeting on Education and Innovation. 


\section{Executive Summary of the Low-Energy Nuclear Physics Town Meeting}

Low-energy nuclear physics, which addresses the origin and properties of atomic nuclei, is a core component of nuclear science. Current and potential future discoveries from this field are compelling, and have relevance to many branches of science, energy, medicine, national security, and technology. Opportunities to advance this field play a critical role in attracting and training the next generation of science leaders needed by industry, national laboratories and academia.

The intellectual challenges for low-energy nuclear science can be organized around the following fundamental questions from the 2012 NRC Decadal Survey of Nuclear Physics:

- How did visible matter come into being and how does it evolve?

- How does subatomic matter organize itself and what phenomena emerge? Are the fundamental interactions that are basic to the structure of matter fully understood?

- How can the knowledge and technological progress provided by nuclear science best be used to benefit society?

Answers to these questions require an even deeper understanding of atomic nuclei both theoretically and experimentally than we have now. The last decade already saw enormous progress in understanding of the nucleus and its role in the cosmos. New ideas, combined with new experimental techniques, major leaps in computing power and impressive improvements in experimental capabilities resulted in discoveries leading to quantitative and qualitative changes in our understanding of nuclear and astrophysical phenomena. This progress is reason for great optimism in the future of low-energy nuclear science in moving forward on the path of understanding the atomic nucleus rooted in fundamental forces. This path requires new insights from experiments with rare and stable isotopes, which will be used to guide theoretical approaches by either confirming predictions or uncovering missing physics. The ultimate goal of this effort is to have a robust and reliable model of nuclei and nuclear reactions with predictive power and quantified uncertainties, coupled with experimental determinations of important properties of key nuclei that allow for example to know the fusion rates of light nuclei, understand the fission patterns of heavy nuclei, trace the origin of the elements in the cosmos, provide nuclear information crucial for the interpretation of experiments involving nuclei such as neutrino-less double-beta decay, searches for dark matter, improve diagnosis and treatment of disease, and contribute in a major way to the nation's stockpile stewardship mission. Achieving this goal involves developing predictive theoretical models that allow the understanding of phenomena associated with small-scale many-body quantum systems of finite size and the reactions of these systems with each other.

In its 2012 Decadal Survey of Nuclear Physics, the National Research Council outlined the impressive accomplishments of the field in the last decade, finds, and recommends:

Finding: The Facility for Rare Isotope Beams is a major new strategic investment in nuclear science. It will have unique capabilities and offers opportunities to answer fundamental questions about the inner workings of the atomic nucleus, the formation of the elements in our universe, and the evolution of the cosmos.

Recommendation: The Department of Energy's Office of Science, in conjunction with the State of Michigan and Michigan State University, should work toward the timely completion of the Facility for Rare Isotope Beams and the initiation of this physics program. 
In view of the unprecedented opportunities the low-energy nuclear science community formulated at the town meeting the following recommendations for the Long Range Planning process:

1. The study of atomic nuclei is the core of nuclear science. The frontier of this field lies in the new opportunities and intellectual challenges offered by the Facility for Rare Isotope Beams (FRIB) ability to produce intense beams of rare isotopes. This ability will lead to an unprecedented understanding of nuclei, of their role in the cosmos, and studies of fundamental interactions. The field is ideally positioned, as well, to advance applications in medicine, energy, national security, and materials science. The health of this field is required to train the talented national workforce needed to assure continuing societal benefits in these critical areas. FRIB provides an essential and unparalleled opportunity to pursue compelling science and maintain world leadership in this field.

- We reaffirm in the strongest possible terms the scientific vision of FRIB and endorse the recommendation laid out in the 2012 National Academy Decadal Study for the timely completion of this advanced rare ion beam facility and the initiation of its full scientific program. Once completed, FRIB will be the world-leading nuclear physics facility that enables tremendous discovery potential for the physics of nuclei, nuclear astrophysics, and the study of fundamental symmetries, with the added benefit of significant applications potential in many areas of societal importance.

Since the 2007 Long Range Plan (LRP), and specifically since realization of its second recommendation of constructing FRIB as the highest priority for new construction, the low-energy nuclear science community has focused its efforts in theory and experiment towards the long-term scientific goals of FRIB. It has enthusiastically promoted the vision that such a facility will provide unprecedented scientific opportunities by opening new vistas for the structure and dynamics of nuclei, and their role in the evolution of the universe, while also delivering in large quantities the isotopes required for testing the fundamental laws of nature and for numerous applications benefitting society. A vibrant research community is an essential part of the FRIB program and the Town Meeting strongly endorsed the need for the broader suite of facilities, theory, and investigator research that is needed to complement FRIB.

2. In support of our science goals, we must continue forefront research to enable new discoveries, to train the next generation of scientists, and to develop new detector and accelerator technologies. Hence:

- We recommend that appropriate funds for operations and near-term upgrades of existing research capabilities at ATLAS, NSCL, university and other facilities be provided. Improvements and developments of instrumentation at existing facilities should be adequately supported. It is essential that strong support for research groups is provided.

- We recommend that enhanced support for nuclear theory be provided to address key questions in nuclear physics and astrophysics and to realize the full potential of the experimental program at FRIB. We recommend the creation of a national FRIB theory center to drive this exciting science and the computational nuclear physics initiative to take maximum advantage of high performance computing critical to this effort.

- To realize the full scientific discovery potential of FRIB and existing facilities it is essential that major experimental systems are available. We recommend:

0 The construction of the $4 \pi$ GRETA detector in a timely manner.

o The timely construction of other new state-of-the-art instruments for FRIB, such as the High-Rigidity Spectrometer and the separator for capture reactions SECAR.

o The construction of ReA12 in a timely manner. 
In preparation for the completion of FRIB and the initiation of its full scientific program, the community has identified a roadmap for the next decade that will ensure the continued intellectual vitality of the field: forefront research at existing U.S. and international facilities needs to stay strong in order to stimulate new discoveries, train the next generation of scientists, develop new concepts, new detectors, and new accelerator technologies. Strong support for research groups is essential to meet milestones on this roadmap. Appropriate funding for operations and near-term upgrades of existing rare isotope and stable beam research capabilities at university facilities and national laboratories (including DOE's premier lowenergy facility ATLAS, the NSF-supported flagship facility NSCL, and JLab, which carries out a unique nuclear structure program with electron beams) represent the means to achieve this goal. The final key elements of the road map are theory, instrumentation, and computing.

Over the last few decades, DOE has established two large nuclear physics user facilities, namely JLab and RHIC. These facilities have enabled outstanding discoveries and in both cases, theory centers strongly coupled to the facility were put in place. The JLab and BNL/RIKEN theory centers are internationally recognized as critical contributors to the success of the associated experimental facility. To realize the full potential of the experimental program at FRIB it is essential that enhanced support for nuclear theory be provided to address key questions in low-energy nuclear science and nuclear astrophysics. To drive this exciting science, the community recommends the creation of a national FRIB Theory Center. The Center will be a key ingredient to the success of the science at FRIB by continuously updating its intellectual framework; optimizing the coupling between theory and experiment; rejuvenating the field by creating permanent positions across the country; attracting young talent through the national FRIB theory fellow program; fostering interdisciplinary collaborations; and shepherding international initiatives. The theory community embraces the positive impact targeted investments in collaborative research efforts can make on advancing specific topics as well as attracting new talent to the field, and recommends similar targeted investments for the future.

In order to realize the full scientific discovery potential of FRIB and continue advances at existing facilities, it is essential that state-of-the-art major experimental systems are available when FRIB starts operation. The low-energy community has developed a plan for targeted investments that will realize the scientific opportunities at FRIB and effectively leverage investments in existing facilities. A highlighted initiative in both the 2002 and 2007 Long Range Plans was GRETA which will be a powerful new instrument needed to accomplish a broad range of experiments that will play an essential role in addressing the intellectual challenges of low-energy nuclear science and nuclear astrophysics. Now that physics campaigns at NSCL and ANL with the $1 \pi$ GRETINA system have demonstrated the successful implementation and unique capabilities of $\gamma$-ray tracking, the top priority is to move forward to completion of the full $4 \pi$ GRETA array. Hence, as was stated in the 2007 Long Range Plan, "Construction of GRETA should begin immediately upon the successful completion of the GRETINA array". The highest priority of the nuclear astrophysics community is for the timely completion of SECAR, the SEparator for CApture Reactions, which is of vital importance in determining many of the key nuclear reaction rates needed to model explosive astrophysical environments. As has been the case at NSCL and similar research institutions, a flagship magnetic spectrograph system will be a centerpiece experimental tool and is essential to achieve the scientific goals of the fast-beam program at FRIB. A need is for a new high-rigidity spectrograph that can bend the extremely neutron-rich beams that FRIB will produce. Hence a new high-rigidity spectrograph is a high priority of the community. Other key equipment opportunities will include; traps for fundamental symmetries measurements, silicon arrays, and a complete decay spectroscopy station.

Much of the vision for FRIB has centered on delivery of reaccelerated beams up to $20 \mathrm{MeV} / \mathrm{u}$ following in-flight separation and stopping. This allows access to research currently not possible at ISOL facilities. To enhance the unique capability of the NSCL there is a tremendous scientific opportunity for the NSF to upgrade the current ReA3 accelerator to ReA12. This upgrade would enable research into the nature of 
pairing in nuclei, how to produce the heaviest possible elements and elucidate the process of fission, among other opportunities. Once upgraded ReA12 would enable a flagship program with FRIB beams in the future. Realization of the science will require new research equipment. The current highest priorities include a recoil separator ISLA, and a HELIOS-like solenoid spectrometer.

Realizing the scientific potential of Low-Energy Nuclear Science demands large-scale simulations in nuclear theory that exploit the US leadership in high-performance computing. Large-scale numerical calculations in theoretical nuclear physics play an increasingly prominent role in our understanding of nucleons, nuclei and their interactions, and provide guidance to present and future experimental programs. The importance of high-performance computing (HPC) in nuclear physics was recognized in the 2007 LRP, and the computational resource and workforce requirements of the overall theoretical nuclear physics program were estimated by the Scientific Grand Challenges panels in 2009. Since the 2007 LRP, nuclear physics has emerged as a driver for exa-scale computing development, and the critical role of HPC in nuclear physics was reinforced in the vision presented in the 2012 National Academy of Sciences report "Exploring the Heart of Matter". During the Computational Nuclear Physics meeting held July 14$15^{\text {th }}$ 2014, the computational resource and workforce requirements of the nuclear physics community have been refined, and newly emerging areas within nuclear physics requiring significant computing resources have been identified. The White paper produced by this meeting details the status of the field and resource needed. The resolutions of the Computational Nuclear Physics meeting were presented and discussed in detail at the Low-Energy Town Meeting following a presentation and subsequent discussion session, which resulted in the following recommendation:

3. We endorse the recommendation of the 2014 Computational Nuclear Physics Meeting "Capitalizing on the pre-exascale systems of 2017 and beyond requires significant new investments in people, advanced software, and complementary capacity computing directed towards nuclear theory."

Education, outreach, and innovation are key components of any vision of the future of the field of Nuclear Science. Our field plays a leading role in education and training of the nation's nuclear science workforce. In addition, our field is ideally positioned to advance applications in medicine, energy, national security, and material science. The health of this field is required to train the workforce needed to assure continuing societal benefits in these critical areas. Continuation of this role is a major goal of the field.

\section{Education and innovation are key components of any vision of the future of the field of nuclear science. We therefore fully endorse the recommendations of the Education and Innovation Town Meeting.}

Progress toward answering the fundamental questions surrounding the nature of nuclear matter relies on a diverse and talented workforce. The same talent pool, with a deep understanding of nuclear physics, is able to address societal problems related to energy, health, and security. Indeed meeting the broad mission of the DOE to "ensure America's security and prosperity by addressing its energy, environmental and nuclear challenges" will rely on experts in the field and a public educated on nuclear science issues. Hence, educational programs in low-energy nuclear science are essential not only for addressing the scientific challenges, but also for meeting societal needs. With adequate support for educational initiatives and forefront research in nuclear science, the highly skilled workforce required for the next decade will be available. Adequate support to do this will include maintenance of forefront research opportunities for undergraduate, graduate students, and postdocs as they are offered by cutting edge accelerator facilities and for increases in support of basic research. The university-based research laboratories have a special role in the educational process as they offer forefront research opportunities, cast a wide net in recruitment, and provide training opportunities over the full depth of the field. 
The needs for education and innovation were discussed at the Town Meeting on Education and Innovation held August 6-8 ${ }^{\text {th }}$, 2014. The outcome of this meeting was a set of recommendations. These were presented and discussed in detail at the Low-Energy Town Meeting following a presentation and subsequent discussion session. The outcome was the above endorsement of the recommendations from the Education and Innovation Town Meeting. 


\section{White Paper: "Low-Energy Nuclear Physics Town Meeting”}

\subsection{Introduction}

In preparation of the 2015 NSAC Long Range Plan (LRP), the two DNP town meetings on Nuclear Astrophysics (NAP), and Low-Energy Nuclear Physics (LENP) were held jointly on August 21-23, 2014, at Texas A\&M, College Station, in Texas. These meetings were co-organized to take advantage of the synergy between fields, as the communities have significant overlap in personnel and the resource needs of both fields are very similar. Approximately 270 participants attended the joint town meetings from national laboratories and a wide range of universities in the United States, Canada, and, in some cases, from overseas. The webpage for the town meetings can be found at: http://www.lecmeeting.org/ and contains the full program along with the slides from individual talks. The talks contain the full content of what was discussed at the town meetings.

The LENP and the NAP communities unanimously endorsed a set of joint resolutions, which are presented and described in the Joint Executive Summary (Section 1) above. The joint resolutions were condensed from the individual recommendations of the two town meetings in order to recognize the highest priorities of the two fields. The recommendations from the LENP town meeting are provided in the Executive Summary in Section 2 above.

Low-energy nuclear science, which addresses the origin and properties of atomic nuclei, is a core component of nuclear physics. Current and potential future discoveries from this field are compelling, and have relevance to many branches of science, energy, medicine, national security, and technology. Opportunities to advance this field play a critical role in attracting and training the next generation of science leaders needed by industry, national laboratories and academia.

The intellectual challenges for low-energy nuclear science can be organized around the following fundamental questions from the 2012 NRC Decadal Survey of Nuclear Physics:

- How did visible matter come into being and how does it evolve?

- How does subatomic matter organize itself and what phenomena emerge?

- Are the fundamental interactions that are basic to the structure of matter fully understood?

- How can the knowledge and technological progress provided by nuclear science best be used to benefit society?

Answers to these questions require an even deeper understanding of atomic nuclei both theoretically and experimentally than we have now. The last decade already saw substantial progress in understanding of the nucleus and its role in the cosmos. New ideas, combined with new experimental techniques, major leaps in computing power and impressive improvements in experimental capabilities resulted in discoveries leading to quantitative and qualitative changes in our understanding of nuclear and astrophysical phenomena. This progress is reason for great optimism in the future of low-energy nuclear science in moving forward on the path of understanding the atomic nucleus rooted in fundamental forces. This path requires new insights from experiments with rare and stable isotopes, which will be used to guide theoretical approaches by either confirming predictions or uncovering missing physics. The ultimate goal of this effort is to have a robust and reliable model of nuclei and nuclear reactions with predictive power and quantified uncertainties, coupled with experimental determinations of important properties of key nuclei that allow for example to know the fusion rates of light nuclei, understand the fission patterns of heavy nuclei, trace the origin of the elements in the cosmos, provide nuclear information crucial for the interpretation of experiments involving nuclei such as neutrino-less double- 
beta decay, searches for dark matter, improve diagnosis and treatment of disease, and contribute in a major way to the nation's stockpile stewardship mission. Achieving this goal involves developing predictive theoretical models that allow the understanding of phenomena associated with small-scale manybody quantum systems of finite size and the reactions of these systems with each other.

The frontier of LENP lies in the new opportunities and intellectual challenges offered by the Facility for Rare Isotope Beams (FRIB) ability to produce intense beams of rare isotopes. This ability will lead to an unprecedented understanding of nuclei, of their role in the cosmos, and studies of fundamental interactions. The field is, as well, positioned to advance applications in medicine, energy, national security, and materials science. The health of this field is required to train the talented national workforce needed to assure continuing societal benefits in these critical areas. FRIB provides an essential and unparalleled opportunity to pursue compelling science and maintain world leadership in this field. These opportunities have been well documented in recent years in a range of publications, including:

- $\quad$ FRIB Opening New Frontiers in Nuclear Science, an FRIB User Community update on the science of FRIB, September 2012

- Nuclear Physics: Exploring the Heart of Matter, National Research Council of the National Academies outlook on the status and future of nuclear science, June 2012

- FRIB Scientific and Technical Merit, providing detail on the FRIB program and required equipment, 2009

- The Frontiers of Nuclear Science, DOE/NSF Nuclear Science Advisory Committee, December 2007

- Four years later: An Interim Report on Facilities for the Future of Science: A Twenty-Year Outlook, DOE, August 2007

- Scientific Opportunities with a FRIB in the United States: National Research Council, December 2006

- $\quad$ Report to NSAC of the Rare-Isotope Beam Task Force, July 2007

- $\quad$ The Science of the Rare Isotope Accelerator (RIA): A Brochure from the RIA Users Community, 2006

In preparation for the completion of FRIB and the initiation of its full scientific program, the community has identified a roadmap for the next decade that will ensure the continued intellectual vitality of the field: forefront research at existing U.S. and international facilities needs to stay strong in order to stimulate new discoveries, train the next generation of scientists, develop new concepts, new detectors, and new accelerator technologies. Strong support for research groups is essential to meet milestones on this roadmap. Appropriate funding for operations and near-term upgrades of existing rare isotope and stable beam research capabilities at university facilities and national laboratories (including DOE's premier lowenergy facility ATLAS, the NSF-supported flagship facility NSCL, and JLab, which carries out a unique nuclear structure program with electron beams) represent the means to achieve this goal. In order to realize the full scientific discovery potential of FRIB and continue advances at existing facilities it is essential that state-of-the-art major experimental systems are available when FRIB starts operation. The low-energy community has developed a plan for targeted investments, as discussed in Section 3.6 that will optimize the scientific opportunities at FRIB and effectively leverage investments in existing facilities. 
Nuclear theory, as discussed in Section 3.2, aims to tie together our understanding of the hadronic interactions arising from QCD with the structure, excitations and dynamics of light to heavy nuclei and nucleonic matter. Nuclei are the core of matter and their properties govern the evolution of the universe and the abundance of the elements. A deep understanding of nuclei and nucleonic matter also allows us to use nuclear physics to probe neutrinos and fundamental symmetries, physics beyond the standard model, and astrophysical environments including supernovae and neutron stars. Developing this understanding is a challenging task because of the diverse energy scales, from the GeV scale of QCD and hadrons to small fractions of an $\mathrm{MeV}$ in the excitation energies of heavy nuclei, and also because of the rich variety of phenomenon important to nuclear physics. A vibrant theory workforce is required to carry out this exciting research program and targeted investments and initiatives are outlined addressing critical needs. In addition, realizing the scientific potential of LENP demands large-scale simulations in nuclear theory that exploit the US leadership in high-performance computing. Large-scale numerical calculations in theoretical nuclear physics play an increasingly prominent role in our understanding of nucleons, nuclei and their interactions, and provide guidance to present and future experimental programs as discussed in detail in Section 3.3.

The NRC Decadal Survey question "How does subatomic matter organize itself and what phenomena emerge?", along with two of the key questions posed in the 2007 LRP: "What is the nature of the nuclear force that binds protons and neutrons into stable and rare isotopes?" and "What is the origin of simple patterns in complex nuclei?", are addressed in Sections 3.4 and 3.5 where the experimental programs in Nuclear Structure and Reactions, and Nature of Dilute and Dense Nuclear Matter and Equation of State are respectively outlined. Experimental data on rare isotopes and stable nuclei under extreme conditions have been and will continue to be the foundation for advancement in these fields. Such information is of crucial importance to both nuclear structure and nuclear astrophysics.

"How can the knowledge and technological progress provided by nuclear science best be used to benefit society?" is illustrated in Section 3.7 where the societal impact of applied nuclear physics research is shown to be ubiquitous, to solve a wide range of "real world" problems, and to dramatically improve people's lives. These efforts play an essential role in national security, medicine, nuclear energy, industry, materials, and education. In addition, credible and reliable nuclear data libraries act as a bridge between science, technology, and society by making the results of basic nuclear physics research available to a broad audience of users, and hence, having a profound effect on the socioeconomic applications of modern nuclear science.

The LENP community plays a leading role in the education and training of the nation's nuclear science workforce. Continuation of this role is a major goal of the field. Indeed meeting the broad mission of the DOE to "ensure America's security and prosperity by addressing its energy, environmental and nuclear challenges" will rely on experts in the field and a public educated on nuclear science issues. Hence, educational programs in low-energy nuclear science are essential not only for addressing the scientific challenges, but also for meeting societal needs. These needs are well documented in the Education and Innovation Town Meeting White Paper and thus not repeated here but were discussed in depth at the LENP meeting. With adequate support for educational initiatives and forefront research in nuclear science, the highly skilled workforce required for the next decade will be available. The support to do this will include maintenance of forefront research opportunities for undergraduate, graduate students, and postdocs as they are offered by cutting edge accelerator facilities and for increases in support of basic research. The university-based research laboratories have a special role in the educational process as they offer forefront research opportunities, cast a wide net in recruitment, and provide training opportunities over the full depth of the field.

It is hoped that the present White Paper will communicate the sense of great anticipation and enthusiasm that came out of the joint town meetings. Over the last decade, the LENP and NAP communities have 
increasingly organized themselves in order to take a coherent approach to resolving the challenges they face. As a result, there is a high level of optimism in view of the unprecedented opportunities for substantial progress. The following chapters, composed by the respective working group organizers, summarize in each of the sub-fields within low-energy nuclear physics the compelling near-term and long-term scientific opportunities, the major accomplishments since the last LRP, the near- and long-term resources needed to achieve these goals, along with the scientific impact and interdisciplinary connections on other fields. The accompanying sister document from the joint nuclear astrophysics town meeting charts out a similar path summarizing the scientific directions and priorities with the field.

In addition to the material presented in this document the community has prepared a number of white papers that contain additional detail. These documents served as important background information for the Joint Town Meetings discussions and the formulation of the priorities presented in the White Paper. They contain a great deal of reference material as well as useful graphical content that will be useful in the Long Range Plan deliberations.

\section{$\underline{\text { ANL ATLAS Strategic Plan }}$}

\section{ARUNA White Paper}

Experimental Equipment Needs for the Facility for Rare Isotope Beams (FRIB)

FRIB Science Update - FRIB Opening New Frontiers in Nuclear Science

FRIB High Rigidity Spectrograph

GRETA White Paper

ISLA White Paper

$\underline{\text { ReA12 Upgrade White Paper }}$

These documents are also available at: http://www.lecmeeting.org/whitepapers.html 


\subsection{Nuclear Theory}

\subsubsection{Introduction}

Nuclear theory aims to tie together our understanding of the hadronic interactions arising from QCD with the structure, excitations and dynamics of light to heavy nuclei and nucleonic matter. Nuclei are the core of matter and their properties govern the evolution of the universe and the abundance of the elements. A deep understanding of nuclei and nucleonic matter allows us to use nuclear physics to probe neutrinos and fundamental symmetries, physics beyond the standard model, and astrophysical environments including supernovae and neutron stars. Developing this understanding is a challenging task because of the diverse energy scales, from the GeV scale of QCD and hadrons to fractions of an $\mathrm{MeV}$ in the excitation energies of nuclei, and also because of the rich variety of phenomena important to nuclear physics. These phenomena include, for example, nuclear structure at the extremes of isospin, reactions at low(astrophysical) and high (accelerator) energies involving light and heavy nuclei, fission of the heavy elements, electroweak transitions and double beta decay in nuclei, and the equation of state and electroweak response in nucleonic matter. These diverse sets of phenomena in nuclei occur in stronglycorrelated quantum system where superfluid pairing, coupling to the continuum, shell closures, and correlations generally all play very important roles.

Theoretical studies related to nuclear structure and astrophysics play a critically important role in tying together laboratory experiments with astrophysical observations. These connections are crucial for understanding the behavior of nuclei in regimes where experiments are either very difficult or impossible to perform, including the most extreme neutron-rich nuclei, neutron star matter, and reactions in unstable systems. The theory of nuclei and their reactions plays an important role across the entire spectrum of U.S. nuclear science. During our town hall meeting we heard many talks on theory relevant to FRIB and also many presentations on parity-violating electron scattering and nuclear correlation studies at JLab, using nuclei to probe hydrodynamic flow at RHIC, applications of light-cone theory at a future electronion collider, to the study of rates and absolute neutrino masses in double-beta decay, and to study neutrino oscillations at FermiLab. Nuclear theorists are also work at the forefront of quantum many-body and reaction theory more broadly, making important contributions in cold atoms and the study of open quantum systems, for example.

Below we summarize progress and opportunities in main areas of the theory of nuclei: ab-initio calculations of nuclei and their reactions, density functional theory, and nuclear astrophysics and highlight connections that extend beyond nuclear science. In each area we review the most important accomplishments since the last long-range plan and highlight the most important challenges that lie directly ahead. The resources required to achieve these goals are very similar in all areas, we conclude with a discussion of needs and how to best achieve the goals of theoretical nuclear science.

\subsubsection{Ab-Initio Nuclear Theory}

Understanding structure and reactions from light to heavy mass and neutron rich nuclei is of fundamental importance in the physics of nuclei and nuclear astrophysics. Microscopic calculations, with controlled and systematic approximations, that start from the basic interactions between nucleons provide us with a valuable tool to connect with experiments at existing and future rare isotope beam facilities such as the facility for rare isotope beams (FRIB) at Michigan State University. In order for these calculations to provide precise and accurate predictions one needs to properly understand (i) two- and many-nucleon forces, (ii) account for open-channels and the influence of continuum degrees of freedom, and (iii) provide error bars on computed observables. 
Effective field theory (EFT) and renormalization group transformations have provided us with a systematic approach to, and a much-improved understanding of the nuclear forces that bind nucleons into stable nuclei and exotic isotopes. This approach is consistent with the underlying symmetries of quantum chromodynamics (QCD), the theory of the strong interaction. The nuclear interaction consists of nucleonnucleon forces at leading order and of three-nucleon forces (3NFs) and forces of higher rank at subleading orders. One of the hallmarks of this approach is the consistent description of currents and forces, with two-body currents (2BCs) being the kin to 3NFs. These developments are providing nuclear theorists with increasingly precise interactions and theoretical uncertainties, which are the basis for accurate predictions. Future challenges are to refine these interactions further and to understand the propagation of uncertainties from the interactions to nuclei and ultimately to the experimental observables. In the optimization of the chiral interactions significant improvements was recently achieved by utilizing optimization software previously used in the optimization of density functionals. Further investigations of the role of order-by-order optimization of the low-energy coupling constants in chiral EFT, and what role three- and many-nucleon forces play in finite nuclei and nucleonic matter is a challenge to be addressed. Implementation of a consistent power-counting scheme in chiral EFT with an investigation of order-byorder regulator and cutoff dependence remains a challenge. The consistent evolution of nuclear forces and observables in the renormalization group needs to be addressed. Other examples are the inclusion of nucleon excitations such as the delta resonance, and the improvement of the optimization process through ideas from statistics and exploitation of the anticipated increase in computational cycles. Another important challenge is to utilize the powerful tools of effective field theory also in halo nuclei and heavier nuclei.

Calculations from lattice QCD have started to bridge the gap between hadrons to nuclei, and are further refining our insights into the nuclear forces. Presently, these calculations employ a pion mass of about $470 \mathrm{MeV}$. Future challenges consist of making a precise link between lattice QCD and nuclear forces, the study of general baryon-baryon interactions and the computation of light nuclei and hyper nuclei directly from lattice QCD. The continuing increase of computational cycles will provide nuclear theorists with the opportunity to meet these goals.

The understanding of light nuclei and their reactions is most advanced, and complementary theoretical methods are available for their description. Examples are the computations of the Hoyle state in ${ }^{12} \mathrm{C}$, based on nucleon-nucleon forces and 3NFs, and the corresponding improvements of our understanding of alpha particle clustering in nuclei like ${ }^{12} \mathrm{C}$ and ${ }^{16} \mathrm{O}$. Precision calculations explore the role of 2BCs in magnetic moments and electromagnetic transitions in light nuclei. Two-nucleon momentum distributions have implications for the EMC effect. For example, a challenge is to improve the calculations by going to higher order in the EFT, and to study cutoff dependence and the role of short range repulsion on saturation properties of nuclei. A challenge is to understand the low-lying excitations and alpha clustering in stable nuclei, and exotic clusterization effects in weakly bound nuclei. Ab-initio approaches can now treat reactions such as ${ }^{3} \mathrm{H}(\mathrm{d}, \mathrm{n}){ }^{4} \mathrm{He},{ }^{3} \mathrm{He}(\mathrm{d}, \mathrm{p})^{4} \mathrm{He}$, and strive to describe binary reaction involving p-shell nuclei and composite projectiles such as ${ }^{7} \mathrm{Li}(\mathrm{d}, \mathrm{p}){ }^{8} \mathrm{Li},{ }^{6} \mathrm{Li}\left(\mathrm{n},{ }^{3} \mathrm{H}\right){ }^{4} \mathrm{He}$ transfer reactions important in fusion research, or ${ }^{3} \mathrm{He}(\alpha, \gamma)^{7} \mathrm{Be}$ relevant for the standard solar model. It can be expected that this approach will solve another longstanding problem, namely a theoretical description of the ${ }^{16} \mathrm{O}(\alpha, \gamma)$ reaction. Further challenges will be studying the influence of 3NF's in reactions with light nuclei in detail.

Key questions in the theory of nuclei concern the limits of nuclear binding, the evolution of shell structure in neutron-rich nuclei, and the emergence of collective phenomena. The understanding of key exotic nuclei will provide us input about entire regions of the nuclear chart. They are relevant for the pathway of rapid neutron capture in astrophysics and input to nucleosynthesis simulations. Weakly bound nuclei magnify less well-known aspects of the nuclear forces, and they exhibit exotic clustering and halo phenomena. In recent years, complementary methods have been developed for the accurate description of 
relevant exotic isotopes that include aspects of weak binding and 3NFs. Examples are our improved understanding of neutron-rich isotopes of oxygen and calcium. Future challenges consist of extending this program to heavier nuclei, to open-shell nuclei, and to making precision calculations of key observables in selected experimentally relevant nuclei. Reliable predictions of relevant dripline nuclei challenge existing many-body frameworks to include coupling to the particle continuum. Another challenge consists of understanding nuclear saturation, relevant ingredient for the nuclear equation of state and the massradius relation in neutron stars, from first principles. Recent calculations starting from chiral interactions have pointed out that it is a challenge to achieve good description of both binding energies and radii of nuclei and nucleonic matter. Although light nuclei might be well described with current chiral interactions, an overbinding of 1-2 MeV per nucleon seems to persist for medium mass and heavy nuclei together with a charge and matter radii that are consistently too small compared to experiment. A challenge is to address this deficiency of current chiral interactions such that one can make accurate predictions of key nuclei at rare isotope beam facilities such as FRIB. The inclusion of many-body forces and continuum effects will require novel ideas and a dramatic increase in computational resources. Thus, the anticipated increase in computational cycles will present nuclear theorists with an opportunity to meet part of this challenge.

Weak decays and probing of fundamental symmetries provide us with a window into physics beyond the standard model. 3NFs has been found to contribute significantly to the long half-life of ${ }^{14} \mathrm{C}$, and the role of 2BCs in weak processes has started to be explored and they explain the quenching of the axial coupling constant $g_{\mathrm{A}}$ in selected nuclei. The nuclear matrix element for neutrinoless double beta decay links the lifetime to the hierarchy and the scale of neutrino masses for Majorana neutrinos. Nuclear theorists are making progress towards the precise and accurate description of weak decays. However, most of the computations of the nuclear matrix elements for this decay have employed phenomenological approaches, and the nuclear matrix element has been found to vary as much as by a factor 2 or 3 in selected nuclei. It is therefore of great importance to provide a precise and accurate computation of this matrix element from first principles. Recent developments and novel extensions of many-body methods has dramatically increased the region of nuclei that can be addressed from first principles, and with the increase in super computing resources this will potentially allow for state-of-the-art computations of weak processes such as neutrino-less double-beta decay in nuclei like ${ }^{48} \mathrm{Ca},{ }^{76} \mathrm{Ge}$ and ${ }^{136} \mathrm{Xe}$. Therefore, it is a major challenge to provide an accurate and precise computation of the nuclear matrix elements for neutrinoless double beta decay in these nuclei.

\subsubsection{Nuclear Reaction Theory}

The understanding of the properties of nuclei and the reactions employed to study them is essential for a complete description of nuclei. Over the last decade models for nuclear reactions have been refined, and new avenues for describing reactions are being pursued in both the reaction and the structure theory community. Several avenues for extending ab-inito structure calculations into the continuum regime are currently being explored and theoretical as well as computational effort will be directed towards fully exploiting those approaches.

Reaction theory is needed to describe elastic and inelastic scattering processes, the fusion of nuclei, as well as the transfer of a nucleon or clusters of nucleons between projectile and target. The complexity of the problem usually requires eliminating possible reaction channels from explicit consideration by introducing effective interactions. The underlying dynamics of the remaining degrees of freedom can then be properly treated with methods of few-body dynamics.

This includes the advanced treatment of breakup channels in transfer reactions, especially for nucleon transfers to weakly bound or unbound (continuum) states. Transfer reactions that populate resonances 
need to be revisited and properly described in order to allow for spectroscopic information to be extracted. An accurate description of reactions that involve both the disintegration of the projectile and the subsequent fusion of one or both fragments with the target nucleus is needed.

The effective interactions between clusters of nucleons that allow a treatment of the reaction dynamics with few degrees of freedom are usually optical potentials, which can be either phenomenologically or microscopically derived. Though they have a long history, they recently received renewed attention. The availability of a wide range of experimental data, coupled with current computational capabilities, has led to greatly improved parameterizations in the phenomenological approaches, in particular for nucleonnucleus reactions. The development of optical potentials for reactions that involve deuterons and other light ions is making much-needed progress. Further improvements are needed to achieve better accuracy and to describe reactions with isotopes away from stability. This must include deformation and nonlocality effects in the potentials, and possibly revising codes that use them as input. However, in order to reliably treat regions away from stability microscopic optical-model approaches are needed, which incorporate information obtained from structure calculations.

Reactions involving the capture of neutrons, protons, and other charged light ions play an important role in the laboratory and in astrophysics. The calculation of cross-sections require reliable optical potentials. To improve the description of direct-capture processes, nuclear deformation needs to be treated consistently, and the contributions from semi-direct processes need to be considered.

Capture proceeding via the formation of a compound nucleus is described in a statistical model, typically a Hauser-Feshbach approach. Multiple Hauser-Feshbach codes for the description of compound-nuclear reactions (including capture) are publicly available, well documented, supported, and user-friendly. These codes require nuclear structure input (discrete levels, level densities, $\gamma$-ray strength functions, fission barriers, etc.), optical potentials as well as pre-equilibrium descriptions. Current codes make use of available nuclear-structure data bases and include a range of phenomenological models to provide the required inputs. The past decade has seen a move towards using microscopic approaches for calculating the necessary inputs. Continuing this effort is very important for achieving more reliable predictions for unstable isotopes. These potentially significant improvements include an explicit treatment of deformation effects, more emphasis on a fully quantum-mechanical description of pre-equilibrium processes, and a better understanding of correlations between different reactions channels.

Low-energy binary reactions proceeding through isolated resonances are described by R-matrix theory. Typically, measured cross sections are fitted by adjusting phenomenological R-matrix parameters, a procedure that allows the extraction of resonance properties and extrapolation to energy regimes for which no data are available. State-of-the-art codes have implemented multi-level and multi-channel treatments. However, improvements need to include the use of calculated structure information and extensions that treat transfer reactions that involve resonances.

More generally, the interplay between direct reactions, semi-direct processes, pre-equilibrium contributions, and compound reactions are not sufficiently understood. For example, the influence of doorway states, simple configurations that couple the reaction entrance channel to more complex configurations, needs to be further explored, and the usually employed energy averaging needs to be revisited. A better understanding of these issues will allow more reliable predictions of cross sections. This is particularly significant when describing low level-density regions away from stability, but will also impact stable nuclei. Efforts need to be made to include theoretical uncertainties with reaction calculations to have a better handle on the predictive power of the theoretical models. Furthermore, the interplay of direct and compound processes is not sufficiently understood for both, inelastic and transfer reactions. These shortcoming will affect the interpretation of radioactive-beam experiments that aim at 
extracting nuclear structure information as well as indirect measurements that aim at determining compound cross sections.

\subsubsection{Density Functional and Other Theories for Heavy Nuclei}

Because of the enormous configuration spaces involved, the properties of medium-mass and heavy nuclei are best described by the superfluid nuclear density functional theory (DFT) rooted in the self-consistent Hartree-Fock-Bogoliubov (HFB), or Bogoliubov-de Gennes, problem. DFT is a quantum mechanical method used in physics and chemistry to investigate the detailed structure of complex many-body systems. The main idea of DFT is to describe an interacting system of fermions via its density rather than its many-body wave function. In doing so, the number of degrees of freedom is dramatically reduced, thus enabling many practical applications. Modern global implementations of nuclear DFT offer a level of accuracy typical of phenomenological approaches based on parameters locally fitted to the data.

The main ingredient of nuclear DFT is an effective two-body density-dependent interaction between nucleons that generates the energy density functional (EDF) through an averaging process. Since the nuclear many-body problem involves two kinds of fermions, protons and neutrons, the EDF depends on isoscalar and isovector densities and currents. The self-consistent HFB equations allow one to compute the nuclear ground state and a set of quasi-particles, which are elementary degrees of freedom of the system; those can be further used to construct the excited states of the system. Since the atomic nucleus is an open system having unbound states at energies above the particle emission threshold, the energy spectrum of HFB quasi-particles contains discrete bound states, resonances, and non-resonant continuum states.

The application of high-performance computing, modern optimization techniques, and statistical methods has revolutionized nuclear DFT during recent years, in terms of both optimizing the EDF and carrying out advanced applications. The method has provided a consistent description of a broad range of phenomena, ranging from global nuclear properties to collective excitations such as rotational states. The ability of DFT to describe nuclear data globally within an approach containing a dozen or so parameters is the major asset of this method. Recent highlights include: large-scale surveys of global nuclear properties that enabled predictions on nucleonic driplines with quantified uncertainties (around 7,000 nuclei with $\mathrm{Z}$ up to 120 are expected to be particle bound); demonstration that continuum-driven correlations can dramatically impact shell structure of exotic nuclei (here, calcium isotopes beyond ${ }^{60} \mathrm{Ca}$ will provide excellent arena where to confront $a b$-initio and DFT predictions); elucidation of shell structure in superheavy nuclei; and fully-self-consistent description of rotational excitations in triaxial nuclei.

The quality of theoretical predictions essentially depends on the quality of model input. In a parallel effort, therefore, methodologies have been developed to validate nuclear EDFs by optimizing their lowenergy coupling constants to experimental data on finite nuclei and pseudodata on nuclear matter and other relevant systems. This enabled the development of EDFs optimized to nuclear and neutron star properties, and to large deformations to describe fission. One of the main challenges of EDF optimization is to find the most relevant data that can tightly constrain the parameter space of the model. This requires a careful analysis of the contribution of each term of the functional to low energy nuclear properties. By means of such studies, it has been realized that the isovector properties of EDF are not well-constrained by the current data. FRIB, with its extended isospin reach, has the potential to dramatically improve the situation. The use of advanced statistical tools has made it possible to uncover parameter interdependences and study correlations between observables thus significantly enhancing the feedback between experiment and nuclear modeling. 
Over the last few years, with the influx of high-quality experimental data on exotic nuclei with significant neutron excess, it has become evident that the standard functionals are too restrictive when one is aiming at detailed quantitative description and extrapolability. An important goal in research in low-energy nuclear physics is to develop a spectroscopic-quality universal nuclear EDF that can be used to explain and predict static and dynamic properties of atomic nuclei. Consequently, various strategies have been devised to develop realistic nuclear EDFs. These include (i) using the density matrix expansion technique to relate the functional to low-momentum interactions obtained systematically within the effective field theory; (ii) extending EDFs by adding higher-order terms in the local densities and currents; and (iii) considering finite range and non-locality. Again, new data from FRIB will inform these developments.

Static, or single-reference, DFT provides an excellent tool for investigating nuclear binding energies and other ground-state properties across the nuclear landscape. In certain cases, it also can be used to treat dynamical processes. A reliable description of excitation/decay and reactions, however, usually requires methods that go beyond static DFT. Since a direct treatment of the nuclear time-evolution is not always possible, one often employs extensions of DFT (usually referred to as "beyond-DFT" methods). The simplest extension, the quasi-particle random phase approximation (QRPA), can be viewed as an adiabatic approximation to the linear response in time-dependent DFT. It provides the entire spectrum of excitations with the same EDF used in static DFT. QRPA has been applied to describe low-energy multipole strength, with a focus on neutron-rich nuclei, in which low-energy strength develops. The important highlight arising from the application of QRPA was the realization that the neutron skin and isovector electric dipole polarizability are strongly correlated, and both can inform the density dependence of symmetry energy. Another important application of QRPA has been in the area of beta decay (especially in the context of astrophysical r-process) and double-beta decay (determination of nuclear matrix elements).

DFT-based methods that go beyond the adiabatic approximation are also now in use. The Generator Coordinate Method is based on multi-reference DFT. It has been applied to systematic surveys of lowlying collective states, shape coexistence, and neutrinoless double-beta decay (when parent and daughter nuclei have markedly different mean fields). Symmetries broken on a mean-field level can be restored by means of projection techniques. This strategy has been successfully used to assess the isospin mixing corrections for superallowed beta decays. Spontaneous fission was studied within nuclear DFT by minimizing the collective action integral employing realistic collective mass. Strong dynamical effects have been predicted due to the interplay between level crossing dynamics, symmetry breaking effects, and nuclear superfluidity.

The time-dependent DFT (TDDFT) and related computational techniques have been successful in making quantitative parameter-free predictions of cross sections in heavy-ion fusion reactions. The superfluid variant of TDDFT was applied to a classic problem of Coulomb excitation and photoabsorption. The tools of superfluid DFT and TDDFT have been applied to a large number of fermionic systems and phenomena: vortex structure in neutron matter and cold atomic systems, generation and dynamics of quantized vortices and their crossing and reconnection, excitation of the Anderson-Higgs modes, the Larkin-Ovchinnikov-Fulde-Ferrell phase, as well as quantum shock waves and excitation of domain walls.

Another perspective on structure of heavy nuclei is offered by symmetry-based approaches. Rather than tackling the nuclear problem from the microscopic perspective, one can focus on a complementary view of the atomic nucleus as a mesoscopic system characterized by shapes, oscillations, and rotations, and described by symmetries applicable to the nucleus as a whole. In this way, properties and regularities, which might not be explicit in a description in terms of individual nucleons or nucleonic densities, are highlighted, providing insights that can inform microscopic understanding. The mesoscopic approach is motivated by the recognition of, and search for, regularities and simple patterns in nuclei that signal the 
appearance of many-body symmetries and associated emergent collective behavior. The recent development in this field is recognition of new partial- and quasi-dynamical symmetries, in which important symmetry remnants persist in systems with otherwise severely broken symmetries. Linking symmetry-based descriptions to microscopic theory remains a challenge, but holds promise for overcoming the computational limitations current $a b$-initio approaches face when approaching heavier nuclear systems.

The prospects for this area of nuclear theory, discussed by the Working Group, include: (i) Developing predictive and quantified nuclear energy density functional rooted in first-principles theory; (ii) Unifying the fields of nuclear structure and reactions; (iii) Providing the microscopic underpinning of observed, and new, (partial-) dynamical symmetries and simple patterns; (iv) Developing predictive microscopic model of fusion and fission that will provide the missing data for astrophysics, nuclear security, and energy research; (v) Predictive and quantified calculations of nuclear matrix elements for fundamental symmetry tests in nuclei and for neutrino physics.

\subsubsection{Nuclear Theory and Nuclear Astrophysics}

Nuclear theory plays a critical role in the study of neutron stars, supernovae, and related astrophysical environments because the extreme ambient conditions that characterize these ultra-dense compact objects cannot be accessed in terrestrial experiments. Theory allows us to reliably extrapolate from modern experimental results at facilities like FRIB, JLab, and RHIC to these extreme environments. Recent developments in theory and astrophysical simulations have begun to unravel how the properties of neutron-rich nuclei and dense nuclear matter shape extreme astrophysical phenomena such as supernova, gamma-ray bursts, $\mathrm{x}$-ray bursts, neutron star mergers and related transient phenomena from accreting and magnetized neutron stars. The equation of state (EOS) of dense matter, its thermal and transport properties, and the neutrino and related weak interaction rates in extreme nuclei and nuclear matter influence key observables: x-ray and gamma rays from neutron star bursts and transients; and neutrinos, gravitational waves, and nucleosynthetic yields from supernova and neutron star mergers.

A quantitative understanding of the interplay between many-body forces and non-perturbative effects in nuclei and dense matter is emerging though advances in nuclear theory and computational nuclear physics. This is largely because modern potentials based on effective field theory (EFT) naturally include an estimate of theoretical errors and provides a consistent framework to include many-body forces and couplings to external fields and currents. This, combined with advances in nuclear many-body theory, Quantum Monte Carlo methods, and access to high-performance computing have led to important breakthroughs. The EOS of uniform dense neutron matter at zero temperature, and the many-body response functions relevant to neutrino interactions rates have been calculated using ab-initio theory. These calculations motivate and define future work to better pin down the role of non-perturbative effects and many-body forces in neutron-rich nuclei and dense matter. The challenge now is to extend these theoretical and computational methods to calculate the properties of asymmetric matter containing a small admixture of protons, heterogenous phases of dense matter encountered in the neutron star crust, and include the effects due to superfluidity, superconductivity and finite temperature. This will require developments in EFT to extend the current potentials to higher density will rely on a better understanding of power counting in the momentum expansion, three-body forces, and the role of the Delta-isobar and advances in QMC, self-consistent Green's function methods, and many-body perturbation theory.

Simulations of supernova, neutron star mergers, x-ray and gamma-ray phenomena involving accreting and magnetized neutrons rely on an input EOS, thermal and transport properties, and weak interaction and neutrino interaction rates that span a vast range of density, temperature and composition. Here, models based on mean field theory with phenomenological interactions and density functional theory will 
continue to central play. They remain the only practical method to cover the full range of ambient conditions encountered in astrophysics. Theoretical developments to improve the nuclear energy density functional using insights from $a b$-initio theory and experiment are necessary. These improved models are also critical to interpret and motivate terrestrial experiments in intermediate and heavy nuclei, and to explore correlations between nuclear structure measurements and specific neutron star properties.

Models to interpret bursts and transient phenomena observed in accreting neutron stars and magnetars rely on the properties of the neutron star crust. Matter in the crust is at sub-saturation density $\boldsymbol{\rho}<10^{14}$ $\mathrm{g} / \mathrm{cm}^{3}$ but is nonetheless characterized by a rich phase structure in the inner crust where exotic spherical and non-spherical (sometimes called pasta) neutron-rich nuclei coexists with neutron matter in a phase that is both solid and superfluid at low temperature. To describe the ground state properties and dynamics of such heterogeneous matter, computational many-body methods such as QMC, (time-dependent) density functional theory, and molecular dynamics need to be extend to handle large numbers of particles, band structure effects in the neutron wave function and long distance Coulomb interactions. Recent calculations of the thermal, mechanical and transport properties, and the non-equilibrium nuclear reaction and neutrino cooling rates have identified the key questions to address and directions for future work. By combining large scale microscopic computations and effective field theory at low temperature, future work can calculate more reliably the superfluid gap, the low energy spectrum of collective excitations, correlations functions needed to determine the transport properties. The extension of these methods to larger temperatures is necessary to describe matter in the density range $10^{10} \mathrm{~g} / \mathrm{cm}^{3}<\rho<10^{14} \mathrm{~g} / \mathrm{cm}^{3}$ encountered in supernova and neutron star merger is also within reach and is an important direction for future research. Interestingly, its been realized recently that such hot heterogeneous matter can also be realized in low energy heavy-ion collisions in the laboratory, and these theoretical methods can be applied to interpret such experiments.

Advances in transport theory and intermediate energy heavy-ion phenomenology are critical to extract useful information about the high-density EOS from heavy-ion collision experiments. These experiments are the only means of creating nuclear matter at higher than normal nuclear densities in the laboratory and can provide information on the nuclear equation of state, both at supra-nuclear and sub-nuclear densities. Given that those collisions progress through different stages, from compression to decompression and to fragmentation, and given that extrapolation is needed for the thermodynamic quantities, from elevated temperatures to low, a careful modeling of the reactions is required within transport theory. There are benefits in employing the same framework consistently for the simulations and the extrapolation, but that requires a compromise between the level of realism and feasibility of reaction simulations. The modeling of fragmentation has close links with modeling of pasta in neutron-star crust. In the near future, a significant narrowing of the correlation between physical inputs and observables for the simulation models of the collisions is needed, such as through extensive model cross-comparisons, for the same physics inputs. One area for which the narrowing of that correlation will be important is the mapping out of the symmetry energy at high density. To progress, nuclear transport theory must advance in realistic description of cluster production, such as further exploiting the time reversibility of fragment production and break-up processes. Currently nuclear transport theory is predominantly semiclassical in nature and should gradually transition to quantal, to close the gaps between the current description of energetic central reactions and the descriptions of peripheral and low-energy central reactions and of nuclear structure. The limits beyond which the current semiclassical transport breaks down are not very well defined. Different options exist for advancing to practical quantal transport, from the non-equilibrium Green's functions, through a diffusion process in an over complete basis of states for the collisions, to a set of Schrodinger equations for the systems, with stochastic potentials.

The recent discovery of a 2 solar mass neutron star implies that the high density EOS is stiffer (has a larger pressure at a specified density) than simple models of high density matter that predict a phase transition to matter containing hyperons, meson condensates and de-confined quark matter. To ascertain if 
a stiff EOS rules out such phase transitions it is important to refine these models by accounting for effects of correlations in the ground state. The competition between the attractive two-body and repulsive threebody hyperon-nucleon forces in high density matter is an area of active research where we can anticipate important progress. Here, ab-initio methods, phenomenology of hypernuclei and lattice QCD methods to directly extract the hyperon-nucleon and hyperon-hyperon phase shifts and hyperon-nucleon-nucleon interaction will improve the state of the art and these advances yield new insights on the maximum mass of neutron stars. Models of strongly interacting dense quark matter and field theoretic methods to directly calculate the EOS at finite chemical potential from QCD using resummation techniques can also yield useful insights about the high density EOS ad the neutron star maximum mass. Complimentary information is contained in the transport properties of these novel high density phases where calculations show that they can be distinct from those expected in nuclear matter, and further work in this area can enable using transport phenomena in neutron stars to either discover or disfavor the existence of phase transitions in the neutron star core.

\subsubsection{Connections to All of Nuclear Science and Beyond}

The theory of atomic nuclei as a strongly-interacting quantum many-body problem is intimately tied to other parts of the nuclear science program and to a diverse range of problems beyond nuclear science. In the high-energy short-distance regime, the nuclear interaction arises directly from QCD, lattice methods are in the early stages of providing input to both the two- and three-nucleon interactions. These interactions provide the basis of quantum many-body calculations of atomic nuclei.

Nuclei are also used as a probe of neutrino properties and physics beyond the standard model. Neutrinoless double-beta decay is a prime example. The goals of the double-beta decay program are to determine if the neutrino is a Majorana particle and to determine the absolute mass scale of neutrinos. While the existence of double-beta decay implies a Majorana neutrino, the rate and hence the size of the proposed experiment depends crucially upon the relevant nuclear matrix element. This matrix element is rather difficult to compute reliably because it occurs only in moderate to heavy nuclei and it depends upon both low and moderate momentum transfers. A variety of techniques including QRPA and the shell-model have been used to address the matrix element, but the remaining uncertainties are rather large. Some of the uncertainties are directly tied to our understanding of the quenching of the axial current in standard beta-decay. With increased knowledge of two-nucleon currents and correlations, and a rapidly-increasing ability to calculate atomic nuclei from fundamental interactions, now is an excellent time to invest more resources in the understanding weak processes such as neutrinoless double-beta decay.

Nuclear response at higher momentum transfer is critical to understanding the scattering of neutrinos by nuclei, as quasi-elastic neutrino scattering is an important ingredient in the oscillations of accelerator neutrinos. These measurements have been valuable to understand the neutrino mixing matrix, and are also crucial for future measurements of $\mathrm{CP}$ violation in the neutrino sector. Two-nucleon currents and correlations have been found to play a crucial role in inclusive electron scattering, and further investigations of the axial currents in nuclei are clearly required to understand, for example, the differences between neutrino- and anti-neutrino scattering from nuclei. Correlations are also important in more exclusive channels. Brookhaven and JLab have probed neutron-neutron (nn) versus neutron-proton (np) correlations in nuclei by examining back-to-back outgoing pairs in hadronic and electron scattering measurements. These measurements demonstrate a large enhancement in np versus pp or $\mathrm{nn}$ crosssections in back-to-back kinematics in ${ }^{12} \mathrm{C}$. These enhancements are directly tied to two-nucleon processes through calculations of momentum distributions. As in the inclusive response, two-nucleon processes are much more important in the neutron-proton pairs. 
Parity violating electron scattering, another weak process, is also being used to probe neutron distributions in nuclei. While the nuclear charge distribution can be obtained from unpolarized electron scattering, the distribution of neutrons has proven more elusive. The neutron distribution can be studied in parity violating electron scattering in elastic kinematics due to the strong coupling to neutrons. Firstgeneration experiments in $\mathrm{Pb}$ have been completed, and further experiments are planned in the calcium isotopes. Such measurements, when performed with sufficient accuracy, provide a stringent test to our understanding of neutron-rich matter.

Research at the intersections of nuclear and astrophysics has an exciting future with the construction of FRIB, enhanced observations of neutron stars including their mass and the mass-radius relation. This neutron-rich matter is also critical to our understanding of astrophysical transients including neutron star mergers and core-collapse supernovae. Understanding nuclear matter in these exotic regimes is crucial to understanding both the origin of the elements, whether they are created in neutron star mergers or corecollapse supernovae, and also the equation of state of cold dense matter and the properties of neutron stars. Further microscopic investigations of the equation of state including studies at finite proton fraction and finite temperature are warranted. Eventually we need a much better quantitative understanding of matter and its electroweak response to enable a better understanding of astrophysical environments and the origin of the elements. Theory and simulations at the microscopic level must be directly tied to astrophysical simulations of compact objects to have the maximum impact.

Atomic nuclei are also important in understanding QCD in the extremes. RHIC is currently measuring flow parameters for a variety of nuclei. The rich variety of atomic nuclei, including isotopic and shape dependence, provide a much richer environment for testing our understanding of the quark-gluon plasma and its viscosity. Similarly, the proposed EIC may probe neutron partonic distributions and coherent processes in deep inelastic scattering. In all these cases an improved understanding of the nuclear structure is required to take maximum advantage of the experiments and theoretical developments in QCD.

The influence of nuclear theory extends well beyond nuclear science. Our ability to understand stronglycoupled quantum systems have proven very effective in other contexts, particularly in cold Fermi atoms. Nuclear theorists have attacked these problems in both the few- and many-body physics governing coldatom systems. Theories of few-nucleon dynamics have included novel applications of Efimov physics to understand dynamic processes like loss rates in cold atomic systems. In many-body physics, nonperturbative techniques have been used to identify and precisely calculate universal constants governing the equation of state, superfluidity and the pairing gap, the density functional, and shear viscosity. Each of these has important analogues in nuclear science, for example dilute neutron matter for the equation of state and superfluidity, and the viscosity in the quark-gluon plasma. Nuclear theorists have also been able to calculate dynamic properties in cold atom systems, including vortices and vortex dynamics. These are the first quantum simulations that were able to follow the dynamics of quantum vortices, including their formation and subsequent evolution. These dynamics also have nuclear analogies, here in the crusts of neutron stars.

These connections extend well beyond cold-atom physics. Nuclear experiments often explore open quantum systems, these studies can include Efimov physics, threshold anomalies, spectral fluctuations and statistics, and clusterization. Each is an important avenue for investigation in nuclear science but also in the wider context of few- and many-body quantum theory.

Nuclear theory and computational nuclear physics provide the links that tie together nuclear science, connecting QCD with nuclear structure, connecting nuclear structure to astrophysical objects and nucleosynthesis, and connecting nuclear properties with fundamental symmetries and neutrino physics, 
electron scattering and heavy-ion experiments. Theory and computation are particularly critical for FRIB, where we want to directly tie nuclear experiments to astrophysical objects and a quantitative understanding of nuclear matter and nucleosynthesis.

\subsubsection{Resource Requirements}

The theory community identified several critical needs to achieve the goals outlined in this document. A major need is a highly-qualified workforce at all levels, including students, postdocs, faculty/staff in physics and support for computer scientists and applied mathematicians for the computational effort required for this science.

A major driver is the new data on very neutron-rich nuclei that will come from FRIB. This data will severely constrain models of nuclei and matter at the extremes of isospin. Additional data from other experiments, including parity violating electron scattering at JLab, and from astrophysical observations, is critical to obtaining a coherent picture of nuclear structure and dynamics. A vibrant theory workforce is required to carry out this research program.

Building upon the successes of the nuclear theory program, as enhanced by investments and initiatives (INT, topical collaborations, inter-disciplinary connections, international programs, and TALENT), we recommend two new initiatives to ensure the experimental program reaches its full potential. We endorse an FRIB theory center, a national program including bridge positions and FRIB fellows, ensuring the highest impact for FRIB science and embracing the full diversity of the FRIB program, tying it to simultaneous advances in nuclear physics and astrophysics.

We also strongly endorse the computational nuclear physics initiative. Key ingredients of this initiative include investments in SciDAC and related initiatives needed to maximize the impact of the experimental program, development of a workforce bridging physicists, applied mathematicians and computer scientists, and deployment of capacity computing to fully exploit leadership class computers. 


\subsection{Low-Energy Computational Nuclear Physics}

\subsubsection{Overview}

Large-scale numerical calculations in theoretical nuclear physics play an increasingly prominent role in our understanding of nucleons, nuclei and their interactions, and provide guidance to present and future experimental programs. The importance of high performance computing (HPC) in nuclear physics (NP) was recognized in the 2007 Long Range plan (LRP), and the computational resource and workforce requirements of the overall theoretical NP program were estimated by the Scientific Grand Challenges panels in 2009 (Young, 2009). Since the 2007 LRP, NP has emerged as a driver for exascale computing development (HPCwire), and the critical role of HPC in NP was reinforced in the vision presented in the 2012 National Academy of Sciences (NAS) report "Exploring the Heart of Matter":

- High performance computing provides answers to questions that neither experiment nor analytic theory can address; hence, it becomes a third leg supporting the field of nuclear physics

This was accompanied by the consequent recommendation:

- A plan should be developed within the theoretical community and enabled by the appropriate sponsors that permits forefront computing resources to be exploited by nuclear science researchers, and establishes the infrastructure and collaborations needed to take advantage of exascale capabilities as they become available.

Two meetings of computational nuclear physicists have been held recently, the first in 2012 (Computational Nuclear Physics 2012) and second in 2014 (Computational Nuclear Physics 2014). During these meetings the computational resource and workforce requirements of the NP community, identified by the Scientific Grand Challenges panels, have been refined, and newly emerging areas within NP requiring significant computing resources have been identified. The Whitepapers produced by these meetings, detailing the status of the field and resource requirements, are publicly available from the meeting websites. The present Working Group (WG) report is a summary of the conclusions and recommendations from these Computational Nuclear Physics (CNP) meetings and of the WG participants.

In preparation for the LRP, in addition to the CNP Whitepaper (Burrows, 2014), a Recommendation and Request, both unanimously supported, emerged from the 2014 CNP meeting:

Recommendation: Realizing the scientific potential of current and future experiments demands largescale computations in nuclear theory that exploit the US leadership in high-performance computing. Capitalizing on the pre-exascale systems of 2017 and beyond requires significant new investments in people, advanced software, and complementary capacity computing directed toward nuclear theory.

Request: To this end, we ask the Long-Range Plan to endorse the creation of an NSAC subcommittee to develop a strategic plan for a diverse program of new investments in computational nuclear theory. We expect this program to include:

- new investments in SciDAC and complementary efforts needed to maximize the impact of the experimental program;

- development of a multi-disciplinary workforce in computational nuclear theory;

- deployment of the necessary capacity computing to fully exploit the nations leadership-class computers;

with support ramping up over five years towards a level of around \$10M per annum. 


\subsubsection{Introduction}

The structure and dynamics of nuclei represent challenging computational problems because naïve meanfield theories based upon the underlying interactions do not provide a quantitative description of their structure. The nuclear interactions are strongly spin- and isospin-dependent, and finely tuned to provide a weak nuclear binding of approximately $8 \mathrm{MeV}$ per nucleon, much smaller than a typical scale in QCD. Large-scale computations have already enabled significant progress in understanding nuclei from the underlying nuclear interactions. The structure and energy levels of the lightest nuclei (consisting of up to of order 12 nucleons) are well reproduced with realistic two-nucleon interactions plus modest threenucleon interactions. The same interactions provide good descriptions of electroweak form factors, transitions and response, as well as nucleon-nucleon correlations. While low-energy microscopic calculations give confidence that a consistent picture of structure and dynamics is emerging, studies of systems containing 12 or so nucleons are only the beginning. Progress in large-scale nuclear calculations is currently being extended to larger nuclei. In heavy neutron-rich nuclei, pairing energies can be comparable to shell closure effects, a regime quite different from the most stable nuclei. The binding energies and electroweak transition rates of these nuclei are crucial to understanding the production of the heaviest elements. In addition, understanding nuclei is critical in exploring fundamental physics, including, for example, the absolute mass scale of neutrinos as to be determined in neutrinoless double beta decay.

The strong nuclear force between nucleons (and also hyperons) originates from quantum chromodynamics (QCD), which governs the interactions of quarks and gluons that are basic constituents of the observable matter in our universe. At low energies, the theory becomes formidable and efforts to theoretically determine fundamental nuclear physics phenomena directly from QCD have met with limited success. A long-standing effort of the U.S. Department of Energy's (DOE) Nuclear Physics program is to understand how QCD in this low-energy regime manifests itself into observed nuclear phenomena, and further, how QCD can be used to make reliable predictions for processes that cannot be experimentally accessed. Lattice quantum chromodynamics (LQCD) currently provides the only ab-initio method for performing QCD calculations in the low-energy regime, and for acquiring a quantitative description of the physics of nucleons and nuclear forces. While the low-energy two-nucleon interactions are well known from precise experimental measurements, the three-nucleon and higher-body interactions are relatively imprecise. Development of methods for calculating multi-nucleon interactions from QCD in conjunction with experiments at FRIB aimed at refining effective models of nuclear interactions is essential for advancing QCD treatments of nuclei and nuclear reactions. During the last decade, significant progress has been made in using the numerical technique of Lattice quantum chromodynamics (LQCD) to calculate multi-nucleon systems directly from quantum chromodynamics (QCD), including nucleon-nucleon and hyperon-nucleon scattering, and the binding of light nuclei. Through a series of calculations, LQCD will provide a determination of the three- and four-nucleon chiral interactions that are required to further refine theoretical predictions of nuclear reactions and nuclear structure, and will complement the low-energy NP experimental program.

\subsubsection{Near-Term and Long-Term Scientific Opportunities}

Large-scale NP computations are dramatically increasing our understanding of nuclear structure and reactions, and of the properties of nuclear matter. In light nuclei, ab-initio calculations of reactions, including those for fission and electroweak processes, are being used to systematically reduce uncertainties and to predict experimentally inaccessible data and processes. Ab-initio approaches are being extended to reactions on medium-mass nuclei. Density Functional Theory (DFT) and its extensions are being applied to medium-mass and heavy nuclei, including neutron-rich systems, nuclear matter, and 
the structure of neutron star crust. The suite of computational methods, including Quantum Monte Carlo (QMC), Configuration Interaction (CI), Coupled Cluster (CC), and DFT, now scale efficiently to the largest computers. These studies will have a direct impact on the experimental NP program. Computational studies of the strongly correlated matter found in nuclei and neutron stars impact the current experimental programs at NSCL and ATLAS, and guide the future program at FRIB. Computations of the neutron distribution in nuclei and of electroweak process are key to the interpretation of results at JLab, and of light-ion thermonuclear reactions that are important to the program at NIF. Precise calculations of nuclear matrix elements provide crucial input for the interpretation of fundamental interaction (FI) experiments. Finally, accurate solutions of the strongly interacting quantum many-body systems will yield new insights and the ability to calculate phenomena, processes, and states of matter that are difficult or impossible to measure experimentally, such as the crust of a neutron star or the core of a fission reactor.

QCD is the underlying theory of the strong interactions that, together with the electroweak force, describes the structure of nuclei and the nature of their constituent hadrons. The powerful numerical technique of LQCD calculations enables key properties of the world around us to be computed from first principles. Thus, such calculations can determine how the fundamental quarks and gluons of QCD give rise to the observed protons, neutrons, pions, and the other hadrons and the chiral nuclear forces among them. The emergence of the nuclear force from QCD can be resolved, and first-principle calculations can be made for light nuclei at the physical values of the quark masses. LQCD calculations have a key role both in predicting the outcomes of, and guiding development of, future experiments, and in fully capitalizing on the current and future DOE experimental nuclear physics programs. In collaboration with traditional nuclear many-body techniques, LQCD studies of chiral nuclear forces will provide a firstprinciples QCD underpinning to studies of nuclear structure and reactions and to nuclear astrophysics, both areas which are central to the FRIB experimental program, and provide data for key interactions, such as those of hypernuclei, for which there is a glaring paucity of empirical data.

A central goal of NP is the detailed explanation of the origin of the elements and of their isotopes. Overwhelmingly, these elements are produced in stars, either during quiescent thermonuclear burning stages, or explosively, as in core-collapse and thermonuclear supernovae. Most of the elements up to the "iron peak", named for the prominent peak in the solar abundances centered on iron, are ejected in supernova explosions, and an understanding of stellar explosions and stellar evolution is central to an understanding of the abundance pattern of the nuclei around us. Stellar evolution calculations involve both nuclear ratio rates generated either theoretically or through experiment, and three-dimensional turbulence and magnetic interactions. Stellar explosions are always multi-dimensional requiring state-ofthe-art radiation/hydrodynamical simulations. As a consequence, addressing this key NP goal of the origin of the elements entails large-scale numerical simulations employing the latest computational tools and the exploiting the most powerful supercomputers. The astrophysics of neutron-rich nuclei is one of four scientific pillars of FRIB. Comparisons of supernova calculations with flagship experimental observations at the Intensity Frontier of the DOE High Energy Physics (HEP) program could reveal new physics beyond the Standard Model.

\subsubsection{A Selection of Focused Objectives:}

Reactions That Made Us: Triple-Alpha Process and ${ }^{12} \mathrm{C}(\alpha, \gamma){ }^{16} \mathrm{O}$. The HPC that will become available during the next few years will enable the first precise calculation of the triple-alpha and ${ }^{12} \mathrm{C}(\alpha, \gamma){ }^{16} \mathrm{O}$ rates for stellar burning. These reactions are critical building blocks to life, and their importance is highlighted by the fact that a quantitative understanding of them is a 2010 U.S. Department of Energy milestone. The thermonuclear reaction rates of alpha-capture on ${ }^{8} \mathrm{Be}$ and ${ }^{12} \mathrm{C}$ during the stellar helium determine the carbon-to-oxygen ratio with broad consequences for the production of all elements made in subsequent burning stages of carbon, neon, oxygen, and silicon. These rates also determine the sizes of the iron cores 
formed in Type II supernovae, and thus the ultimate fate of the collapsed remnant into either a neutron star or a black hole. Therefore, the ability to accurately model stellar evolution and nucleosynthesis is highly dependent on a detailed knowledge of these two reactions, which is currently far from sufficient.

A comprehensive understanding of the mechanism behind these two key reactions, and the ability to model the chemical evolution of the universe, will be obtained. Success will permit an accurate determination of the reaction rates at low energies relevant to stellar burning, which are currently limited by large experimental uncertainties. In particular, the uncertainty in the ${ }^{12} \mathrm{C}(\alpha, \gamma){ }^{16} \mathrm{O}$ reaction rate is currently about $40 \%$. At the same time, ab-initio tools will be developed to describe the structure of weakly bound nuclei that will be studied at the FRIB. Verification of model predictions by experiments at FRIB will provide necessary checks on the theoretical approaches and the underlying two- and threebody forces used.

Chiral Nuclear Forces from QCD. Insufficient knowledge of the three-nucleon interaction is responsible for the largest systematic uncertainties in nuclear structure and reaction calculations of light nuclei. Without a better knowledge of this interaction, absolute binding energies and level orderings of excited states of nuclei cannot be calculated with high fidelity. LQCD calculations during the next few years will remove this obstacle by providing direct calculations of the multi-nucleon forces from QCD. These LQCD calculations will complement efforts in the nuclear structure community, providing them with important theoretical inputs, and will also complement the FRIB experimental program.

Light Nuclei from QCD. For the first time, the binding energies of the lightest nuclei will be calculated directly from QCD at the physical values of the light-quark masses and including electromagnetism. Along with the binding energies, basic properties of nuclei will be calculated, such as magnetic moments, matrix elements of the electroweak operators and their gluonic structure. Currently, such calculations are being performed for s-shell nuclei at unphysical quark masses that yield a pion with mass $\mathrm{m} \sim 400 \mathrm{MeV}$. Coordinating with other LQCD efforts within the US, through USQCD, calculations at the physical quark masses are expected to begin within the next few years, and the inclusion of electromagnetic effects soon thereafter. Extensions to p-shell light-nuclei have not been performed yet, but the required methods are understood, and their development is ongoing.

Physics of Extreme Neutron-Rich Nuclei and Matter. In the crust of neutron stars, neutron-rich nuclei coexist with a surrounding gas of neutrons; the structure and dynamic properties of this unusual matter will be calculated using advanced Monte Carlo methods. In turn, it will be possible to interpret the wealth of astronomical data obtained from visual, x-ray, and $\gamma$-ray telescopes. Details of the nature of these sites, and the processes (such as potentially gravitational wave emission) that occur there, will be inferred. The major computational challenge in these efforts is to develop and implement scalable algorithms for the strongly interacting inhomogeneous quantum many-body problem. An important complement to this work will be the experimental program conducted at the FRIB. The envisioned theoretical and computational tools will provide an essential framework to interpret FRIB experimental data and will eventually guide the future experimental program. In turn, FRIB data will be essential to verify ab-initio calculations and calibrate the nuclear many-body Hamiltonian.

Computations of neutron star matter, when combined with observations, will provide information about nucleonic matter at supernuclear densities. The interpretation of observations of isolated, cooling neutron stars require an accurate microscopic understanding of superfluidity and neutrino emission processes in neutron-rich matter. Similarly, observations of gravity waves with the advanced Laser Interferometer Gravitational-Wave Observatory (LIGO) and future detectors will, when combined with a realistic description of the neutron star matter, allow for the mass and radius of a neutron star to be inferred. 
Combined observations of multiple neutron stars will produce definitive constraints on the equation of the state of the densest matter in the universe.

Microscopic Description of Nuclear Fission. HPC, combined with experiments, will provide a predictive framework for fission that may lead to improved nuclear reactor design. In the area of national security, developing a theoretical description of fission aligns with the goals of the National Nuclear Security Administration (NNSA) Stockpile Stewardship Program, which entails an accurate and complete modeling of the behavior and performance of devices in the nation's aging nuclear weapons stockpile. Improving the accuracy of that description is central to the continuing process of certifying both the safety and the reliability of the stockpile without resumption of nuclear testing and to reduce the threat from nuclear proliferation. Of all the various nuclear decay processes, nuclear fission-important in the rprocess nucleosynthesis, in the modeling of reactions relevant to the advanced fuel cycle for next generation reactors, and in the context of national security - is among the most difficult to tackle. It is a quantum many-body tunneling problem whose typical time-scale changes by orders of magnitude when adding just a few nucleons. The microscopic theory of nuclear fission, rooted in inter-nucleon interactions, remains a particularly difficult challenge.

\subsubsection{Major Accomplishments Since the Last Long Range Plan}

Since the last LRP, significant progress has been made in the nuclear many-body theory for structure and reaction calculations, and in the application of Lattice QCD to low-energy nuclear physics. Some representative accomplishments are:

- In ab-initio calculations of medium-mass neutron-rich nuclei, emphasizing the importance of threenucleon interactions and particle continuum.

- Lattice QCD calculations of the nucleon-nucleon and hyperon-nucleon phase-shifts (extrapolated to the physical pion mass from calculations at unphysical light-quark masses using chiral effective field theory).

- The first ab-initio calculations of important and unique light nuclear states and transitions, including the Hoyle state of ${ }^{12} \mathrm{C}$ and the lifetime of ${ }^{14} \mathrm{C}$.

- Advances in ab-initio studies of fusion reactions, combining the no-core shell model and resonating group techniques.

- Lattice QCD calculations of the spectrum of the lightest nuclei and hypernuclei (at pion masses $\mathrm{m}$ $>300 \mathrm{MeV}$ ), which required the development of algorithms to perform the large number of necessary contractions.

- Improved density functionals (UNEDF0 and UNEDF1) that simultaneously reproduce $a b$-initio calculations of inhomogeneous neutron matter, and significantly advance our abilities to reproduce fission barriers and half-lives.

- Calculations, including uncertainty estimates, of the full range of atomic nuclei, based upon these improved density functionals.

- Lattice QCD calculations of the magnetic moments of the lightest nuclei (at a pion mass of $\mathrm{m} \sim$ $800 \mathrm{MeV}$ ).

- Lattice QCD calculations of the binding of quarkonia to light-nuclei, performed at heavy pion masses, and extrapolated to the physical light-quark.

- Important impacts on other fields, particularly cold atom physics, in both ab-initio and density functional calculations. 


\subsubsection{Near-Term and Long-Term Resource Needs}

\subsubsection{Workforce Needs}

During the last few years, the resources required to accomplish the NP goals, both workforce and computational, have been estimated and refined. CNP bridges a number of areas of science, and as such, the expertise of a broad range of individuals, including physicists, computer scientists, applied mathematicians, is vital to the success of the program. Contributions at all levels from students to senior researchers are important to the large-scale efforts that are necessary. The 2013 Tribble committee report on implementing the 2007 LRP emphasized the role of young scientists:

"People remain the key factor. In particular, early-career scientists working at the interface between nuclear theory, computer science, and applied mathematics are critical to make future impact, especially in the era of extreme computing that demands the novel coding paradigms and algorithmic developments required by novel architectures."

Even with the rapid growth of national computing resources, the overall improvement in NP computing since the last LRP far outstrips this growth. The ingenuity and dedication of the NP workforce, and collaboration with colleagues in computer science and applied mathematics, have led to fundamentally better algorithms and structurally more efficient code bases, allowing computations that at the time of the last LRP we would not have thought possible over such a short timescale. As computational environments become more diverse and more specialized, it is crucial that the workforce include those individuals with skills sufficient to master these challenges, which extend beyond the traditional purview of nuclear theory. The emerging trends in new architectures must be followed, and algorithms and software infrastructure must be developed to enable their exploitation and advance the overall program. In light of the demands on large-scale computation from the NP program, new investment is essential to develop the needed multi-disciplinary workforce in CNP. Interdisciplinary programs, such as SciDAC, will become even more crucial in the future to help foster such collaborations.

Many of the innovations in our field have been borne by young researchers: graduate students, postdoctoral scholars and junior faculty, and we are fortunate that the quality of the young scientists throughout the NP subfields is outstanding. Since the 2007 LRP, many junior faculty and permanent laboratory staff positions have been filled by computationally oriented nuclear theorists, both within the US and abroad. These junior researchers have been successful in acquiring competitive funding for their research, winning numerous early career awards and prizes. Notably, of the 14 DOE Early Career research awards in nuclear theory since the program's inception in 2010, 10 have been awarded to researchers with a strong focus on computational nuclear theory. More broadly, NP plays an important role in training computational scientists to help meet the needs of the DOE workforce and industry. Further strengthening the computational effort in NP will contribute to the broader DOE mission and to society as a whole.

The SciDAC program has enabled significant advances in computational NP within the US in the past decade, precisely because it has brought together leading domain specialists (nuclear physicists) and computational scientists to focus on algorithmic improvement as well as improving domain codes for NP. The SciDAC programs enable the support of a very special class of collaborators - ones not typically covered under base funding. Notably, the algorithmic advances and highly-optimized code development that SciDAC has enabled, have been key to the leveraging of substantial leadership-class computational resources. The SciDAC-funded positions that have been enabled are cost effective and highly leveraged 
as the many domain scientists with which they interact are supported out of NP base funding. For example, the UNEDF SciDAC-2 and NUCLEI SciDAC-3 projects in nuclear structure and reactions brought together a large fraction of the nuclear theory community, and represented a significant increase in support for junior scientists. But this was possible only through a tremendous leveraging of the base programs in NP and ASCR, and strong support from NNSA. In FY13, the historical level of NP funding for SciDAC was reduced by a factor of nearly three; key areas of computational nuclear physics now lack support for the development of the computational infrastructure, with the potential loss of leadership not only in the computational domain, but also in the corresponding experimental and theoretical domains. In addition, scientifically strong SCIDAC-3 proposals in astrophysics and in nuclear structure and reactions were not funded. Present funding is insufficient to ensure a strong future for the field and it is critical to reverse this striking decline.

At present, the SciDAC program provides the bulk of support for software and algorithmic support of computational nuclear physics, but there are needs not covered by SciDAC. For example, there are other nuclear structure and reactions collaborations utilizing significant computing resources, including $\mathrm{NuN}$, TORUS, and SA-NCSM that are not directly supported by SciDAC. There are also other programs that provide, or could potentially provide, additional contributions such as the NSF Software Infrastructure for Sustained Innovation programs, Intel Parallel Computing Centers, and other partnerships with industry. In QCD in particular, the strong partnership with nVidia has led to very effective use of GPUs. In addition, the various DOE computing facilities have programs for postdoctoral positions for candidates working in scientific areas aligned with leadership-class computing. There are and have been similar opportunities at other labs and computational nuclear theory PhDs have proven to be very competitive when seeking these domain-science-related postdoc positions. These smaller scale programs are important and strongly encouraged as they allow more dynamic response to the emerging needs of the field.

NP plays an important role in educating computational scientists to help meet the needs of the DOE workforce. These need and the challenges in meeting them are documented in the July 23, 2014 ASCAC Workforce Subcommittee. Each of the subfields of CNP has identified future manpower needs:

- Investment in the personnel responsible for software development will be critical to ensure a viable LQCD program, and re-assure leadership-class facilities that computational resources provided to the community will be optimally exploited (thereby leading to at least Moore's Law growth in resources). This will require at least the same level of support that was provided to SciDAC-2, and hence a significant increase in the level of support currently being provided to SciDAC-3.

- An additional 10 positions over 5 years is estimated to be needed in nuclear structure and reactions and associated computer science and applied math. Approximately $1 / 2$ of these positions would be nuclear physicists and 1/2 computer science and applied math scientists. These new positions are critical to enable the effort to scale to the largest-scale machines heading toward exascale, and to support the FRIB and related experimental program in a timely manner.

- Astrophysics proposals were not funded by the 2013 SciDAC and, as a result, a number of research groups have had to identify alternate funding for their junior team members in order to maintain scientific viability. This defunding has compromised the United States' ability to realize the progress envisioned in the context of previous DOE investments. It is estimated that approximately 15 junior nuclear astrophysicists and computational scientists are required to reestablish the momentum previously enabled by past DOE support, which is crucial heading through the petascale era.

It needs to be stressed that the ability of NP to obtain computational resources on leadership-class computers from ASCR depends crucially upon having cutting-edge codes and algorithms that will efficiency execute on the newest computational architectures. This requires having a vibrant workforce to 
port existing codes and generate new codes for CNP. Without such a workforce, the field will not be able to acquire the computational resources required to accomplish its mission.

\subsubsection{Computational Resource Needs}

The future program in CNP requires substantial computational resources. Advanced calculations that can resolve the key issues of NP require leadership computing at the largest scales (capability computing), medium scales (capacity computing), and a software infrastructure to effectively exploit that computing. The NP community has aggressively pursued these resources and thereby received substantial allocations on leadership-class computers, such as those under DOE's INCITE program and programs within the NSF and NNSA. It is through the generous access to such capability computational resources, and the recognition of the need for a range of computational capabilities to exploit them, such as the dedicated facilities of USQCD, that such impressive progress has been made. However, as outlined in the NP Scientific Grand Challenges report from 2009 (Young, 2009), substantially increased computational resources are required to capitalize on the scientific potential made possible by CNP. During 2014, approximately 1 Billion CPU core-hours have been made available to all of theoretical CNP within the U.S., including QCD, nuclear structure, and nuclear astrophysics. This corresponds to about 0.1 Petaflopyears, placing nuclear physics at the earliest part of the resource time-lines presented in the Scientific Grand Challenges report. New generations of calculations will require substantially more computing.

Advances in nuclear structure and reactions were made possible by a rapid increase in the ability of the community to use the largest-scale computational resources, and in available computational resources. Large-scale usage in nuclear structure and reactions rose from $80 \mathrm{M}$ core-hrs/year in 2009 to $345 \mathrm{M}$ corehrs/year in 2014, with over 500M core-hrs/year projected for 2015. Nuclear structure and reactions researchers receive considerable resources from INCITE, NERSC, NSF (TeraGrid, NICS), as well as institutional computational centers. The INCITE resources, particularly those at OLCF, have increased rapidly. In 2014, the nuclear theory INCITE was the fourth largest out of 59 awards for 2014. In addition, there is a tremendous need for additional computational resources, both capability and capacity, as indicated by the expected 2015 requests.

A significant fraction of the computational resources for LQCD come from the dedicated capacity hardware operated by USQCD and funded jointly by NP and HEP. In 2014, the total USQCD resources were 468M CPU core-hours/yr and 8.9M GPU hours/yr. For typical present-day LQCD applications, this amount of GPU resources corresponds to 265M CPU core-hours/yr. Currently, approximately half of the total USQCD resources (366M core-hours/yr) are available for NP calculations, including both hot and cold QCD. LQCD researchers also receive considerable resources from INCITE, NERSC, NSF, as well as from institutional computational centers. In 2014, LQCD was the largest INCITE award (340M corehours); the NP part was $100 \mathrm{M}$ core-hours. Thus, the total amount of computer time available for NP LQCD in 2014 is approximately 600M core-hours. In 2014, approximately 50M core-hours were allocated to calculate low-energy nuclear forces from QCD. Contributions to the NP program from NERSC have not been increasing in a way commensurate with Moore's Law, limiting the overall productivity of the NP LQCD effort.

Two examples of the computational resource requirements by NP are shown in Figures 1 and 2. Figure 1 shows the resources required for representative nuclear structure calculations. Calculations of nuclei in the ${ }^{78} \mathrm{Ni}$ region and of static properties of matter in the crust of a neutron star require a computational facility with tens of petaflop-years of capacity, while computations of nuclei in the ${ }^{132} \mathrm{Sn}$ region and transport properties of crust matter require a facility with hundreds of petaflop-years capacity. These computations will allow for the limits of nuclear stability to be determined. This theoretical effort will have a major impact upon the experimental program to search for these limits at facilities such as the 
FRIB. The combination will allow for the modeling of some of the most exotic environments in astrophysics, and understand and model the chemical evolution of the universe.

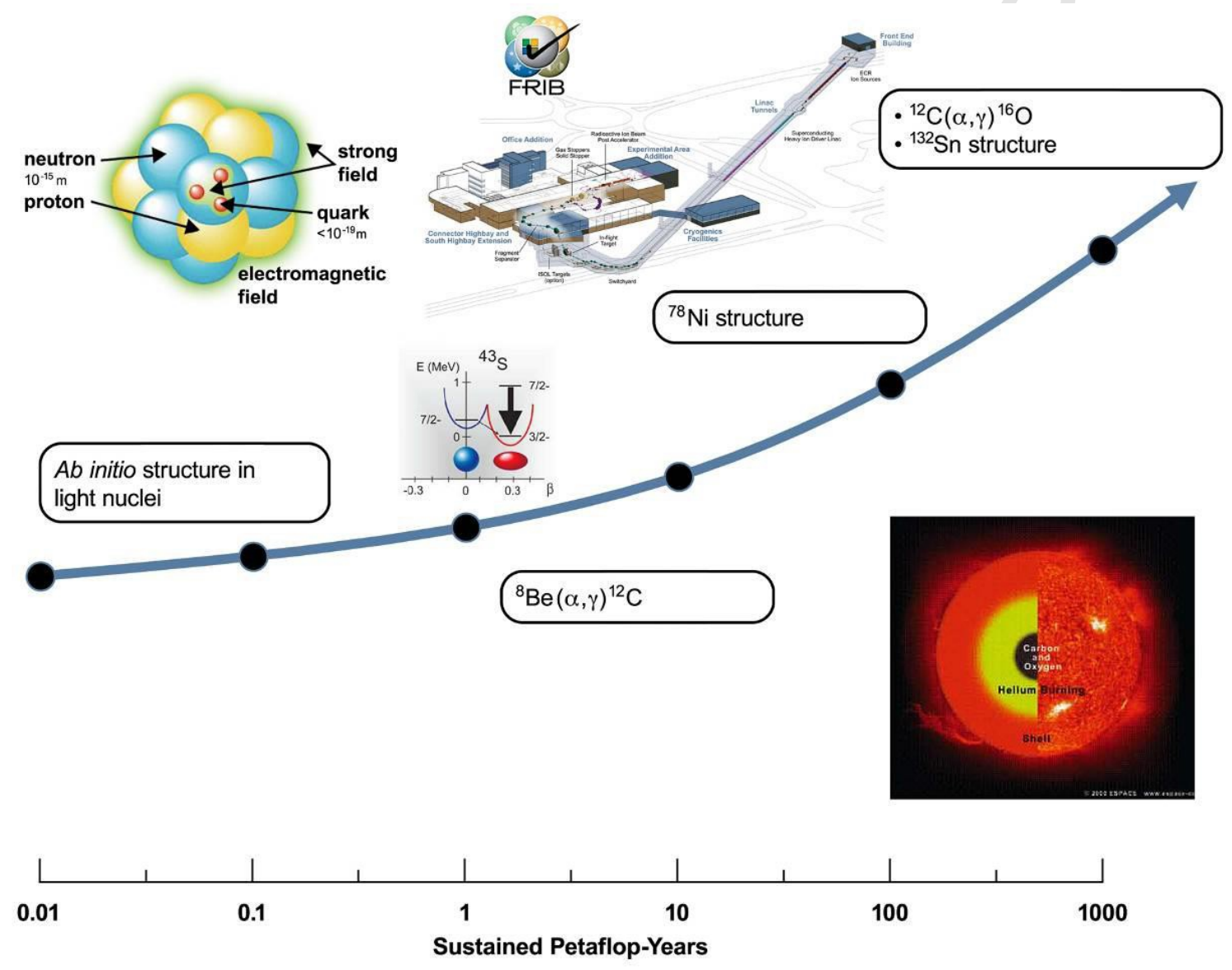

Figure 1. Computational resource requirements for representative nuclear structure calculations. Reproduced from the Scientific Grand Challenges report (Young, 2009).

Figure 2 shows the resource requirements for LQCD calculations of quantities of importance to the structure and interactions of nuclei. With sustained petascale resources, nucleon-nucleon and hyperonnucleon interaction parameters will be computed in the limit of exact isospin symmetry with high precision. With an order of magnitude increase in computational resources, the deuteron axial-charge will be accessible. This is one of the key ingredients constraining certain fusion reactions-within the sun, for example. Further, the three-nucleon interactions, including the three-neutron interactions, will be calculated. Soon after these calculations, and following calculations in particle physics, it is anticipated that these LQCD calculations will be performed with the physical values of the four lightest quark masses and with fully-dynamical QED. Throughout this period, development of EFTs and their overlap with other subfields of nuclear physics will continue and be enhanced. The research impact in this area will come from calculating observables that are currently inaccessible to experiments, and have great relevance not only to the QCD community, but to the broader nuclear physics community such as those of nuclear structure and nuclear astrophysics. 


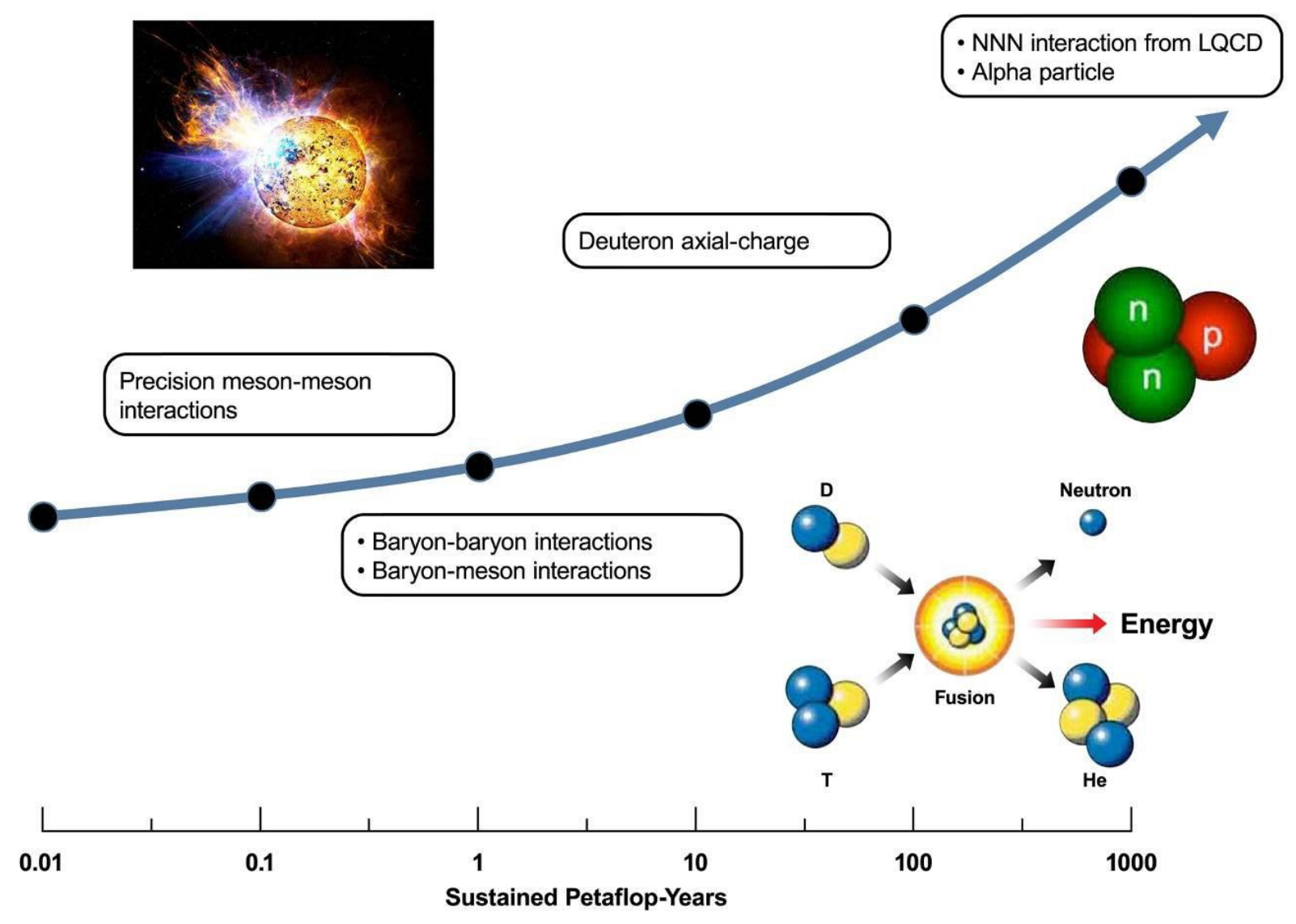

Figure 2. Computational resource requirements for representative Lattice QCD calculations of importance to lowenergy nuclear physics. Reproduced from the Scientific Grand Challenges report (Young, 2009).

In order to accomplish the nuclear physics mission, with experiment and theory optimally complementing each other, substantial increases in the number of nuclear theorists proficient in HPC, in the number of applied mathematicians and computer scientists collaborating with nuclear physicists, and in the available capacity computing resources directed at these calculations, is required.

\subsubsection{Scientific Impact on Other Fields and Interdisciplinary Connections}

The increased computational resources of the past decade have brought various subfields of NP to a point where interdisciplinary research is now a reality. Going forward, no longer will different subfields of NP exist independently of one another, as the level of precision of calculations has reached a stage where continued improvement involves direct collaboration with other areas of physics. Nowhere is this more clearly seen than in the area of nuclear structure and reactions and LQCD. For example, in nuclear structure and reactions, the need for a precise determination of the three-nucleon force can only be satisfied with LQCD calculations. Aspects of the nuclear equation of state used in astrophysics simulations can be determined through LQCD investigations of exotic nuclear systems.

Many of the calculations within the purview of nuclear forces and LQCD will have direct impacts on other areas of physics, such as the standard model of particle physics; e.g. in Dark matter searches and in measuring the properties of neutrinos, for which knowledge of the precise response of nuclei to external probes is crucial. There is a strong symbiosis between the LQCD efforts in NP and in particle physics. 
This includes not only the exploitation of new algorithms and theoretical advances, but also the development of software and computational infrastructure. Sharing gauge-field configurations within USQCD has been vital to fully exploiting the available computational resources. This symbiosis will only strengthen through the petascale era.

Building upon the SciDAC projects, and their link to the SciDAC institutes, collaborations with applied mathematicians and computer scientists will continue to be strengthened going forward. These collaborations, and the funding mechanisms that support them, are essential to the success of the NP program. Without them, the ability to utilize the leadership-class computational resources, and to make optimal use of the capacity computing resources, will be severely compromised. As an example of the impact on other disciplines, with the current SciDAC projects, progress has been made in adapting existing SciDAC Institute software to handle the calculations required for quantum field theory effectively transferring developments in linear algebra from USQCD software to ASCR software.

\subsubsection{Summary}

Given its intriguing nature and importance in the birth of the universe, in astrophysical settings, in energy generation, and in industrial and medical applications, it is fundamentally important and of great practical significance that we have a detailed understanding of this complex quantum many-body system. The theoretical goal of increased predictive power for describing nuclear processes that occur in nature, but cannot be measured in the laboratory with sufficient precision, requires detailed simulations using the next generation of leadership-class computers and beyond, capacity computing resources and cutting-edge algorithms. The amalgam of theoretical, computational, and experimental nuclear physics will continue to transform our understanding of the nuclear forces and nuclei, and finally provide a description of nuclear forces in terms of the fundamental quarks and gluons of QCD. This will allow us to finally understand how the properties of hadrons and nuclei depend on the fundamental (input) parameters of nature. Furthermore, many longstanding questions within nuclear physics - such as the nature of the threenucleon or spin-orbit interaction - will be answered, and systems not accessible by experiment can be ascertained using LQCD. Ultimately, LQCD will have the ability to provide predictive capability in the realm of low-energy nuclear physics that will have direct connections to nuclear structure, nuclear reactions, and nuclear astrophysics, as well as the broader nuclear physics community.

In order to accomplish the mission of nuclear physics, access to increasing computational resources and expertise is critical. Continued access to capability-scale resources on leadership-class platforms is essential to the program. These resources are currently under the purview of ASCR, and it is hoped that fraction of these resources provided to nuclear physics and LQCD remains stable into the future. There is a significantly growing need for capacity computing resources in the community, which will most likely not be provided by ASCR, as the scope of projects increase toward the precision required to impact the experimental program, and as new areas requiring computing emerge. Last, but by no means least, is the need for an enhanced workforce with HPC proficiency that is critical to executing the nuclear physics mission, and the close collaboration with applied mathematicians and relevant computer scientists.

This computational working group unanimously endorses the Recommendation and Request from the Computational Nuclear Physics Meeting in 2014 which are detailed in the opening "Overview" section of this chapter. 


\section{Bibliography}

- Retrieved from HPCwire:

http://www.hpcwire.com/hpcwire/2012-04-12/meet_the_exascale_apps.html

- Computational Nuclear Physics 2012. Retrieved from: http://www.jlab.org/conferences/cnp2012/

- Computational Nuclear Physics 2014. Retrieved from http://www.jlab.org/conferences/cnp2014/

- Tribble NSAC Report. (2013). Retrieved from: http://science.energy.gov/ /media/np/nsac/pdf/20130201/2013_NSAC_Implementing_the_2007 Long_Range_Plan.pdf

- Young, G. (2009). Forefront Questions in Nuclear Science and the Role of Computing at the Extreme Scale. US Department of Energy. 


\subsection{Nuclear Structure and Reactions}

\subsubsection{Introduction}

The 2012 National Academies Decadal Study on Nuclear Physics captured the major scientific challenges for low-energy nuclear physics and nuclear astrophysics in terms of four overarching questions. The relevant NRC question most related to nuclear structure and reactions is: "How does subatomic matter organize itself and what phenomena emerge?" Addressing this compelling challenge requires a comprehensive picture of the atomic nucleus, which describes this strongly-interacting quantum manybody system quantitatively and with predictive power. Such a picture ideally must be rooted in the fundamental interactions at play between its constituents. This research thrust directly addresses two of the key questions posed to the field by the 2007 LRP: "What is the nature of the nuclear force that binds protons and neutrons into stable and rare isotopes?" and "What is the origin of simple patterns in complex nuclei?”

Closely related to studies of nuclear structure is the challenge of describing accurately how nuclei interact with each other. Nuclear reactions represent an essential tool for the extraction of crucial information for both nuclear structure and nuclear astrophysics.

Experimental data on the structure and reactions of rare isotopes and stable nuclei under extreme conditions have been and will continue to be foundations for advancement in this field. Tremendous progress has been made since the 2007 Long Range Plan and the community is ready to take this progress to a new level with FRIB at the horizon.

The following sections address the questions posed to the Working Groups in the framework of the 2014 Town Meeting of the Low-Energy Nuclear Physics and Astrophysics community held at TAMU, College Station, Texas. The response is largely based on community input presented during three sessions on the topics of nuclear structure and nuclear reactions. The presented material can be found online at the town meeting webpage.

\subsubsection{Progress Since the Last Long Range Plan}

Any compilation of highlights from a field as broad as that of Nuclear Structure and Reactions is necessarily incomplete and the successes of the programs outlined below are meant to be representative of the cutting-edge activities ongoing in our vibrant community.

\subsubsection{The Limits of Nuclear Existence}

Key data are often obtained at the limits of the nuclear landscape accessible to experiments at present generation facilities and only with the most advanced equipment and/or the highest beam intensity. That is where new phenomena are uncovered and some of the most sensitive benchmarks for theories attempting to model the nuclear force are posed.

Toward the drip lines - existence, extrapolation, and information along the way: A fundamental question in nuclear science is what combinations of neutrons and protons can be made into a bound system. The limit of how many neutrons a given number of protons can bind is unknown for all but the lightest elements, owing to the delicate interplay between single-particle and collective quantum effects in the nucleus. 
- The discovery of ${ }^{40} \mathrm{Mg}$ and ${ }^{42,43} \mathrm{Al}$ in a tour-de-force measurement at NSCL implied that the neutron drip line is further out than previously anticipated. This is an example of the experimental work needed to understand what combinations of neutrons and protons can make up a nucleus. First data on the structure of ${ }^{40} \mathrm{Mg}$ is now available using a 2-proton removal knockout reaction.

- Measurements of the production of neutron-rich nuclei by the fragmentation of a ${ }^{76} \mathrm{Ge}$ beam were performed at NSCL. The cross sections were determined for a large range of nuclei including 15 new isotopes that are the most neutron-rich nuclides of the elements chlorine to manganese. The cross section systematics pointed to shell evolution effects in the region around ${ }^{62} \mathrm{Ti}$, revealing that production cross section, in fact, may bear nuclear structure information at the extremes.

- First spectroscopy of the neutron-rich nucleus ${ }^{60} \mathrm{Ti}$ was accomplished at NSCL with the GRETINA array coupled to the S800 spectrograph in one proton knockout from ${ }^{61} \mathrm{~V}$ projectiles. First level-scheme and cross section information for ${ }^{60} \mathrm{Ti}$ now are the closest extrapolation points towards ${ }^{60} \mathrm{Ca}$, the critical $\mathrm{Z}=20$ isotope that is key to determining the dripline in the Ca isotopic chain.

- Studies on the proton-rich side of the line of stability pushed boundaries towards nuclei with even larger proton excess. The ground-state proton emitter ${ }^{69} \mathrm{Br}$ and two-proton decay in the doublymagic ${ }^{48} \mathrm{Ni}$ were directly observed for the first time at NSCL. In the case of ${ }^{69} \mathrm{Br}$, critical information was obtained on the proton separation energy of ${ }^{69} \mathrm{Br}$, which has important consequences for the rp-process occurring in type I x-ray bursts.

- Gamma-decay properties of proton resonances in several nuclei along the $\mathrm{N}=\mathrm{Z}$ line, such as ${ }^{31} \mathrm{~S}$ with Gammasphere at ATLAS or ${ }^{58} \mathrm{Zn}$ with GRETINA at NSCL, were determined to further constrain the rp-process path. It is the unique capabilities of the these large $\gamma$-ray arrays which allow for the identification of not only excitation energies but also spins and parities of excited states which are critical in determining reaction rates associated with the rp process.

- Excited states in the $\mathrm{T}_{\mathrm{z}}=-2{ }^{52} \mathrm{Ni}$ nucleus were observed with SeGA and S800 at NSCL and the mirror symmetry was exploited to provide additional evidence for isospin non-conserving interactions. The complete spectroscopy of the $\mathrm{T}=0$ states in the self-conjugate ${ }^{62} \mathrm{Ga}$ nucleus was performed with Gammasphere and FMA at ATLAS providing stringent test for interactions between neutrons and proton.

Beyond the neutron drip line - the example of oxygen: The neutron drip line seems to evolve regularly from light to medium-mass nuclei except for a striking anomaly in the oxygen isotopes, making this chain a key benchmark for nuclear models until higher-Z isotope chains come into reach.

- Spectroscopy of oxygen isotopes at and beyond the neutron drip line - performed at NSCL and confirmed at GSI/Germany - revealed a rich structure at $\mathrm{Z}=8$, and provided conclusive evidence for new magic numbers at $\mathrm{N}=14$ and 16, which impact the location of the drip line in oxygen. The drivers of the modified shell structure can be attributed to key ingredients of the nuclear force that were not quantified before. The important differing parts of the nuclear force are amplified in neutron-rich and weakly-bound nuclei, making them key testing grounds. The data led to the most significant advance in the understanding of the nuclear many-body problem in decades, with tensor-rank interactions, 3-nucleon forces, possibly two-neutron radioactivity and effects of the particle continuum in a sensitive interplay.

The heaviest nuclei: Superheavy nuclei constitute the limit of nuclear mass and charge, and their existence hinges on a delicate balance between the attractive nuclear force and the Coulomb repulsion between protons. The discovery of new elements with the highest atomic numbers has brought much excitement to the atomic and nuclear physics communities. In this period two new elements joined the periodic table, Flerovium $(\mathrm{Z}=114)$ and Livermorium $(\mathrm{Z}=116)$, and measurements to characterize the chemistry of elements $112(\mathrm{Cn})$ and $114(\mathrm{Fl})$ were carried out.

- The discovery of a new chemical element with atomic number $\mathrm{Z}=117$ at Dubna and the confirmation of elements 117, 115, and 113 (GSI), and 115, 113 (LBNL) mark a major advance in heavy element nuclear physics. The measured decay properties show a sharp onset of stability for heavier isotopes with $Z \geq 111$, validating the exciting concept of the long sought island of 
enhanced stability for superheavy nuclei. It should be noted that the target material (Bk) was supplied through the NP Isotope Program.

- Pioneering $\alpha$-, $\gamma$-, and $x$-ray spectroscopy of decay chains originating from element $Z=115$ was performed at GSI and LBNL. The observed $\gamma$-ray transitions provide the first spectroscopic data on excited states of superheavy elements, and pave the way for a new generation of measurements that include unique Z-identification by means of characteristic K-x ray detection, which would be a game-changer in the study of superheavy elements.

- Precision mass trap measurements of $\mathrm{Z}=102,103$ transfermium elements $\left({ }^{252,253,254} \mathrm{No},{ }^{255,256} \mathrm{Lr}\right)$ extend the region of known masses to Ds and Rg isotopes through known $\alpha$ decay chains.

- Prompt and isomeric $\gamma$-decay spectroscopy of transfermium elements has provided detailed information on the nature of single particle and collective degrees of freedom in nuclei around $\mathrm{Z}=104$, and is important to understand ground-state configurations of superheavy nuclei around $\mathrm{Z}=114$.

\subsubsection{Evolution of Shell Structure}

The broad picture of shell behavior of atomic nuclei, including the nuclear potential and the resulting shell structure, has been established in the valley of stability; however, dramatic modifications to the familiar ordering of single-particle orbits in rare isotopes have been found. The driving forces behind the changes can only be isolated in the most exotic nuclei, providing crucial information on the interactions that cannot be gained from stable nuclear species.

Light Nuclei: Light nuclei often pose the most stringent benchmarks for the most advanced, ab-initio type nuclear models. Their spectra are typically fairly simple and have provided deep insights into nuclear structure physics. The availability of light nuclei close to the drip lines has highlighted the role of the particle continuum and clustering.

- Inelastic scattering measurements have revealed that the $\mathrm{N}=8$ shell gap seems to disappear in ${ }^{12} \mathrm{Be}$. However two-neutron correlation measurements following the inelastic scattering of ${ }^{14} \mathrm{Be}$ showed a substantially reduced deformation length in ${ }^{14} \mathrm{Be}$, and a possible restoration of the $\mathrm{N}=8$ shell gap.

- Neutron-transfer to single-particle states in the lightest bound $\mathrm{N}=9$ isotone ${ }^{14} \mathrm{~B}$ showed the evolution of the $1 \mathrm{~s}$ and $0 \mathrm{~d}$ effective single-particle energies, with the $1 \mathrm{~s}_{1 / 2}$ orbital continuing to drop well below the $0 \mathrm{~d}_{5 / 2}$ and a smooth extension of the systematic behavior established from heavier $\mathrm{N}=9$ isotones.

A new Island of Inversion: The Island of Inversion around N=20 has provided much insight into shell evolution. Another region of rapid structural change occurs for neutron-rich $\mathrm{N}=40$ nuclei, with key experimental data only available recently. Fueled by deformation-driving neutron intruder orbitals, $\mathrm{N}=40$ isotones exhibit a remarkable variation in collectivity with a rapid shape change from the spherical to the deformed/collective regime around $\mathrm{Ni}, \mathrm{Fe}$ and $\mathrm{Cr}$, in analogy to the classic island of inversion around ${ }^{31} \mathrm{Na}$.

- Pioneering conversion-electron spectroscopy of ${ }^{68} \mathrm{Ni}$ populated in $\beta$-decay provided the first precise, direct measurement of the isomeric $0^{+}{ }_{2}$ state in ${ }^{68} \mathrm{Ni}$, pointing towards shape coexistence at low excitation energies - a hallmark of Islands of Inversion.

- Excited states of ${ }^{64} \mathrm{Cr}$, the nucleus predicted to be at the center of the new Island of Inversion, were identified for the first time with fast-beam in-beam $\gamma$-ray spectroscopy.

- Collectivity in the $\mathrm{Fe}$ and $\mathrm{Cr}$ isotopic chains up to and beyond $\mathrm{N}=40$ was quantified for the first time with intermediate-energy Coulomb excitation and excited-state lifetime measurements, quantifying the onset of collectivity at $\mathrm{N}=40$ in $\mathrm{Cr}$ and $\mathrm{Fe}$. 
- High-spin spectroscopy with Gammasphere following multi-nucleon transfer reactions of $\mathrm{Cr}, \mathrm{Fe}$ and $\mathrm{Ni}$ at the entrance to the new Island of Inversion tracked the descent of the deformationdriving intruder states in a unique way from their impact on higher-spin states.

The ${ }^{78} \mathrm{Ni}$ region: The region around ${ }^{78} \mathrm{Ni}$ is important to understanding changes in shell structure as well as for the anchoring nuclear properties at the start of the astrophysical r-process.

- Measurements at RIKEN have confirmed and improved upon the lifetime measurement of ${ }^{78} \mathrm{Ni}$ at NSCL, as well as providing five new $\beta$-decay half-lives in the region around ${ }^{78} \mathrm{Ni}$.

- Fast $\beta$ decays near ${ }^{78} \mathrm{Ni}$, and $\beta$ delayed-neutron emission measured at HRIBF impact simulated $\mathrm{r}$ process abundances by speeding up the flow toward heavy nuclei.

The example of doubly-magic ${ }^{132} \mathrm{Sn}$ : Doubly-magic nuclei are the cornerstones of the configurationinteraction shell models - Detailed spectroscopic studies of ${ }^{132} \mathrm{Sn}$, the most neutron-rich doubly-magic member of the long Sn key isotopic chain, confirmed that sometimes magic numbers persist in the exotic regime.

- At HRIBF, using neutron-rich tin beams from ${ }^{124} \mathrm{Sn}$ to ${ }^{132} \mathrm{Sn}$ and the (d,p) and $\left({ }^{9} \mathrm{Be},{ }^{8} \mathrm{Be}\right)$ oneneutron transfer reactions, highlighted the evolution of single-particle states from stability to ${ }^{133} \mathrm{Sn}$ at various angular momentum transfers, proving that $\mathrm{N}=82$ is a good magic number in tin.

- At HRIBF, barrier-energy Coulex measurements of ${ }^{124-128} \mathrm{Sn}$ on a ${ }^{12} \mathrm{C}$ target showed that, as expected, the $\mathrm{B}(\mathrm{E} 2)$ values decrease as the double shell closure at ${ }^{132} \mathrm{Sn}$ is approached, despite the remarkably constant energies of their first $2^{+}$states.

- Above ${ }^{132} \mathrm{Sn}$, transfer measurements involving ${ }^{134} \mathrm{Te}$ beams produced at HRIBF, showed that away from doubly-magic ${ }^{132} \mathrm{Sn}$, single-particle strength fragments quickly.

Hypernuclei as a unique window into nuclear shell structure: The spectroscopy of hypernuclei reveals unique details of the effective nucleon-nucleon $\mathrm{NN}$ and hyperon $\Lambda \mathrm{N}$ forces

- Since the last long range plan, a series of high precision, hypernuclear spectroscopy experiments with light $(\mathrm{A}=7)$ to medium-heavy nuclei $(\mathrm{A}=52)$ have been performed using the $\left(\mathrm{e}, \mathrm{e}^{\prime} \mathrm{K}^{+}\right)$ reaction. These measurements, which made use of the high-quality electron beam at Jefferson Lab, have clearly demonstrated the physics potential of the electro-production of hypernuclei.

\subsubsection{Benchmarking Developments in Nuclear Structure Theory}

Fueled by developments in nuclear theory that push the description of nuclear properties more and more towards high-precision ab-initio models, precise measurements, often on light nuclei, have provided unique benchmarks for ab-initio models or qualified alleged exotic behavior in comparison to welldeveloped configuration-interaction shell models.

\section{The structure of neutron-rich carbon isotopes}

A series of excited-state lifetime measurements carried out at LBNL and NSCL was used to determine the electric quadruple transition rates $\mathrm{B}(\mathrm{E} 2: 2 \rightarrow 0)$ in neutron-rich ${ }^{16,18,20} \mathrm{C}$ isotopes. Complementary data for neutron transfer to ${ }^{16} \mathrm{C}$ demonstrated that the neutron-spectroscopic factors were consistent with shellmodel predictions. Taken together, these data confront previous reports of "decoupled" proton and neutron matter distributions, and provide a sensitive test of theories that aim to describe nuclear structure near to the neutron dripline.

\section{Tests of ab-initio theory from precision measurements}

- Absolute cross sections have been determined following single neutron knockout reactions from ${ }^{10} \mathrm{Be}$ and ${ }^{10} \mathrm{C}$ at intermediate energy. Nucleon density distributions and bound-state wave function overlaps obtained from both variational Monte Carlo and no-core shell-model ab-initio calculations have been incorporated into the theoretical description of knockout reactions, 
providing a unique way to confront density distributions and wave function overlaps calculated from first principles with measured nucleon removal cross sections.

- Excited-state lifetimes for ${ }^{10} \mathrm{Be}$ determined with Gammasphere have put $a b$-initio nuclear models or highly-developed configuration interaction shell models to the test and their results will remain benchmarks for future theoretical approaches to come.

\subsubsection{Correlations in Nuclei}

The nucleus is a strongly-correlated many-body system. Sensitive experiments have been devised to probe nucleon-nucleon correlations in a variety of regimes.

Complex multi-particle decays: Nuclear decays often provide the first information on very exotic systems and offer unique ways to study the correlations of the decay particles.

- The visualization of proton radioactivity and beta-delayed multi-proton emission with information on the correlations of the emitted protons became possible through the use of an optical TPC for proton-dripline fp-shell nuclei produced at NSCL.

- At NSCL, using the Sweeper Magnet and the MoNA-LISA neutron array, the unbound nuclear system ${ }^{16} \mathrm{Be}$ was found to decay by the simultaneous emission of 2 neutrons with di-neutron character, shedding light on possible strong neutron-neutron correlations which ultimately may also be important for an understanding of neutron matter and neutron stars.

- Studies of the two-proton decay of the extremely neutron-deficient isotope of carbon ${ }^{8} \mathrm{C}$ revealed di-proton-like correlations, in contrast to the situation for the two-proton decay of ${ }^{6} \mathrm{Be}$. These results were obtained from the first example of discrete two-step two-proton decays. The work utilized nucleon removal from ${ }^{9} \mathrm{C}$ relied on the properties of the High Resolution Array (HiRA) charged-particle detector at NSCL. The measurements also identified the first example of an isospin-allowed two-proton transition between isobaric-analog states (IAS), with the observation of the decay of the ${ }^{8} \mathrm{C}$ IAS in ${ }^{8} \mathrm{~B}$ to the $\mathrm{T}=10^{+}$excitation in ${ }^{6} \mathrm{Li}$.

- Beyond the neutron separation energy, $\beta 2 n \gamma$ multi-coincidence spectroscopy was employed at HRIBF/ORNL in the discovery of the first large $\beta 2 \mathrm{n}$ decay branch in a fission fragment.

Correlations beyond effective-interaction theory: A reduction in spectroscopic strength relative to shellmodel calculations has been established from (e,e'p) studies and one-nucleon knockout reactions and is attributed to correlation effects - short-range, long-range and tensor - which are beyond the effectiveinteraction theory that forms the basis of most modern shell-model approaches.

- From a large body of one-nucleon knockout reactions, significant additional reduction in spectroscopic strength was observed for the minority nucleon species (e.g., neutrons in a proton rich nucleus), while less reduction was found for weakly-bound neutrons or protons in neutron- or proton-rich nuclei, respectively.

- Consistently analyzed nucleon transfer data, most recently from HELIOS at ATLAS, confirmed the reduction of spectroscopic strength observed close to stability.

- The spatial correlation of two like nucleons were studied with direct $2 \mathrm{n}$ removal from proton-rich nuclei and 2p removal from neutron-rich nuclei - always leading to nuclei more exotic than the projectile. It was shown that the residue parallel momentum distributions in these reactions offer a clear spectroscopic signal of the angular momentum of the pair of nucleons removed, and thus of the residue final-state spins and spectroscopy of the typically very exotic knockout residues.

The nature of short-range and tensor correlations with high-energy electromagnetic probes:

- At Jefferson Lab, using electro-magnetic probes to knock out nucleons from the nucleus provides valuable insight into nuclear structure. In particular, recent measurements done in kinematics that are inaccessible to free, stationary nucleons, researchers have finally isolated the signal for studying initial-state tensor and short-range correlations in the nuclei as well as studying the highmomentum components of the deuteron. 
- Nucleon-nucleon correlations in the nucleus - Many fields in nuclear and particle fields require precise knowledge of nuclear structure in order to extract their physics. This is perhaps most strikingly seen in deep inelastic scattering measurements on nuclei where per nucleon ratios of deuterium to heavy nuclei are not simply unity. Recent ab-initio calculations as well as phenomenological results have shown that an understanding of these results requires knowledge of nucleon structure and in particular knowledge of the tensor and short-range correlations that occur between nucleons in a nucleus.

\subsubsection{Symmetries and Simplicities in Complex Systems}

Astonishingly simple patterns in the level schemes and for the relations between transition matrix elements are observed even for nuclei with more than 100 nucleons. These are often signatures of symmetries of the nuclear many-body system that allow for near-parameter-free predictions of some nuclear properties for heavy nuclei where computational power is limiting the applicability of configuration-interaction shell-model or ab-initio type models

- Dynamical symmetries - or spectrum generating algebras - that allow describing nuclei as vibrators, axially symmetric rotors and axially-asymmetric, gamma-soft rotors, have long provided predictions for the properties of nuclei that are outside the realm of microscopic models that attempt to solve the many-body problem. Only very few nuclei seem to follow closely the predictions of dynamical symmetries. However, only recently, the benchmarking of partial dynamical symmetries vs. data by Casten et al. has shed light on the validity of these algebraic approaches and has shown that these partial symmetries may be applicable to a broader range of nuclei.

- Very regular rotational bands in the excitations of well-deformed nuclei have long been studied. A new phenomenon, octupole phonon condensation, related to octupole correlations and rotation of octupole-deformed nuclei, was recently confirmed experimentally at ANL.

- Gammasphere data from ATLAS on ${ }^{157,158}$ Er pushes beyond the rotational band termination and reveals a return to collectivity at ultra-high angular momenta, establishing a record for the highest spin structure ever observed.

- Using both in-beam and decay data, a very detailed mapping of excited states has been performed for several $N=90$ nuclei in the rare-earth region. A large number of rotational bands have been identified in these nuclei built upon both vibrational and quasiparticle excitations. What is unique about these data is that many of the observed bands have a partner excitation that can be found at an excitation energy relative to its partner that is constant for all partner pairs. While shape coexistence is a general phenomenon in nuclei, the two classes of states have very similar deformations, and thus, the underlying explanation for the co-existence is not well understood.

\subsubsection{Spin-Isospin Structure of Nuclei}

Charge-exchange reactions have long been a unique tool to study the spin-isospin response of nuclei. In contrast to studies of $\beta$-decay, where the Q-value limits accessible states, charge-exchange reactions can probe the entire response function, including the region of giant resonances

- Probes in the $\beta^{+}$and $\beta^{-}$direction, $\left({ }^{7} \mathrm{Li},{ }^{7} \mathrm{Be}+\gamma\right)$ and $(p, n)$, were developed and implemented to extend charge-exchange reactions to rare-isotope beams in inverse kinematics.

- Configuration mixing in the near-dripline nucleus ${ }^{12} \mathrm{Be}$ and in ${ }^{34} \mathrm{Si}$ at the entrance to the $\mathrm{N}=20$ Island of Inversion were probed with charge exchange reactions for the first time and provided complementary information in these regions of rapid shell evolution around $\mathrm{N}=8$ and $\mathrm{N}=20$. 


\subsubsection{Collectivity in Nuclei}

A remarkable feature of medium- and heavy-mass nuclei is the emergence of novel collective properties that are not apparent in few-body systems. Novel inverse-kinematics techniques have been developed to probe collective degrees of freedom also in rare isotopes.

- Sn isotopes, Coulomb excitation below the barrier - At HRIBF, Coulomb excitation of 124, 126,

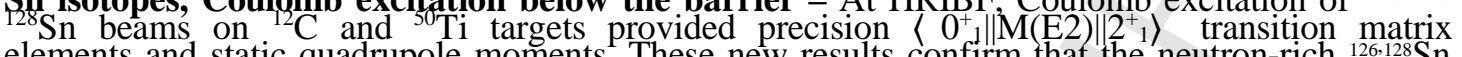
elements and static quadrupole moments. These new results confirm that the neutron-rich ${ }^{126,128} \mathrm{Sn}$ isotopes have deformations consistent with zero. The study marked the first report on measured $2_{1}$ static quadrupole moments for a closed-shell rare isotope.

- Sn isotopes, Intermediate-energy Coulomb excitation - The $\mathrm{B}\left(\mathrm{E} 2,0^{+}{ }_{1} \rightarrow 2^{+}{ }_{1}\right)$ value for ${ }^{104} \mathrm{Sn}$ for the most neutron-deficient $\mathrm{Sn}$ isotope yet was measured, indicating that the most modern many-body calculations remain unable to describe the enhanced collectivity below mid-shell in Sn approaching ${ }^{100} \mathrm{Sn}$. The enhanced collectivity is attributed to proton particle-hole configurations beyond the presently limited shell-model spaces, with the asymmetry of the $B(E 2)$ value trend around mid-shell to originate from enhanced proton excitations across $Z=50$ as $N=Z$ is approached.

- Shapes along the $\mathbf{N}=\mathbf{Z}$ line - The transition rates from the yrast $2^{+}$and $4^{+}$states in the selfconjugate ${ }^{72} \mathrm{Kr}$ nucleus were studied via lifetime measurements employing the GRETINA array with a novel application of the recoil-distance method. The large collectivity observed for the $4^{+} \rightarrow 2^{+}$transition suggests a prolate character of the higher excited states on top of an oblate ground state, pointing to a rapid shape change at low energies within ${ }^{72} \mathrm{Kr}$.

\subsubsection{Precision Masses}

Penning traps have provided the most precise masses for stable and rare isotopes. The deduced nucleon separation energies are often the most basic benchmarks for nuclear models

- At FSU, using precision, cryogenic, Penning-trap techniques with two simultaneously-trapped, triply-charged ions we have measured the atomic masses of ${ }^{115} \mathrm{In}$ and ${ }^{115} \mathrm{Sn}$ to better than $0.2 \mathrm{ppb}$ fractional precision. Combined with the energy of the $3 / 2^{+}$first excited state of ${ }^{115} \mathrm{Sn}$, the $\mathrm{Q}$ value of the ${ }^{115}$ In $9 / 2^{+}$to ${ }^{115} \mathrm{Sn} 3 / 2^{+} \beta$ decay was confirmed to have the smallest $Q$ value of any known $\beta$ decay.

- A new long-lived isomeric state in ${ }^{65} \mathrm{Fe}$ was discovered with Penning trap mass spectrometry with the Low-Energy Beam and Ion Trap Facility at the NSCL. For the new isomer in ${ }^{65} \mathrm{Fe}$ an excitation energy of 402(5) $\mathrm{keV}$ has been determined from the measured mass difference between the isomeric and ground states.

- The masses of neutron-rich Calcium isotopes out to ${ }^{54} \mathrm{Ca}$ are now known with high precision from highly sensitive measurements performed at TITAN/TRIUMF and ISOLDE/CERN. The deduced neutron separation energies constitute formidable benchmarks for ab-initio type models that manage to predict the structure in semi-magic Ca isotopes.

\subsubsection{Neutron Skins}

The neutron-skin thickness in heavy nuclei is linked to the equation of state of neutron-rich matter and hence to the properties of neutron stars. Reactions with nuclei with thick neutron skins may be the only way to study very neutron-rich matter in the laboratory.

- While elastic scattering measurements can easily determine the charge distribution of the protons in the nucleus, it is extremely difficult to determine the neutron distributions in nuclei in a model independent way. Parity violating electron scattering provides a unique solution to this problem 
and a pioneering experiment was recently performed to determine the neutron skin thickness of ${ }^{208} \mathrm{~Pb}$.

\subsubsection{Nuclear Reactions}

Nuclear reactions play a crucial role in developing our understanding of nuclear structure. In order to extract quantities that can be compared with and guide theory, we must understand nuclear reactions. A proper understanding of reaction theory is also vital in the planning of experiments.

Scattering studies in support of transfer-reaction theory: While studies of transfer reactions have provided a far better understanding of the nuclear shell model approaching the limits of stability, the interpretation of results from these measurements still rely on reaction theory. Great strides have been made, but much remains to be done.

- Guided by detailed neutron and proton scattering data, Dispersive Optical Models have provided a link between nuclear structure and nuclear reactions.

- Studies of fission following neutron transfer and comparisons with neutron-induced fission have been used to begin validating theories of surrogate reactions and compound-nucleus formation following neutron capture.

\section{Nuclear Fusion:}

- Towards the heaviest nuclei: Predicting cross sections for the synthesis of the heaviest elements requires the understanding of the different aspects of the reaction - capture, formation, and survival. Studies of highly excited ${ }^{274} \mathrm{Hs}$ produced in hot fusion reactions revealed a high survival probability in a regime where the fission barrier is largely due to shell effects. The understanding of survival probabilities is critical for planning future searches for super-heavy elements.

- Light-heavy-ion fusion: New studies of the fusion of light-heavy ions such as ${ }^{12} \mathrm{C}+{ }^{12} \mathrm{C}$ at extreme sub-barrier energies have revealed surprising enhancements in the fusion cross section in comparison to the predictions of conventional fusion theory. Similar measurements are now being conducted using rare-isotope beams, in some cases with extremely neutron-rich projectiles such as ${ }^{20} \mathrm{O}$. These results are important for understanding cluster-burning in massive stars, and may provide new insights into the processes at work in the crusts of neutron stars.

- Fusion of very neutron-rich nuclei: In fusion reactions induced by radioactive ${ }^{132} \mathrm{Sn}$ and ${ }^{130} \mathrm{Te}$ beams on $\mathrm{Ni}$ targets, the fusion enhancement through nucleon transfer reported previously in lighter systems was not observed, providing a formidable benchmark for theory to describe fusion enhancement across all projectiles.

Multi-nucleon transfer reactions (MNT): Multi-nucleon transfer reactions (or deep inelastic scattering reactions) between heavy ions are a versatile tool to probe many aspects of nuclear structure. They have been used to obtain new information about exotic nuclei at high spin that are inaccessible otherwise. Many of these studies have been performed at ATLAS using Gammasphere.

- Previously, MNT reactions identified evidence for the $\mathrm{N}=32$ shell closure in $\mathrm{Ca}, \mathrm{Ti}$ and $\mathrm{Cr}$ nuclei. These studies have continued and have been extended to $\mathrm{Ni}$ isotopes around $\mathrm{N}=40$. These studies have allowed for identification of excited states associated with both prolate and oblate shapes in ${ }^{66,68,70} \mathrm{Ni}$ co-existing with the spherical ground states. These results can be compared directly with shell model calculations which attempt to describe the "island of inversion" identified for neutron-rich $\mathrm{Cr}$ and Fe isotopes in the proximity of $\mathrm{N}=40$.

- MNT reactions have been used to study high-senority excitations in the even ${ }^{118-128} \mathrm{Sn}$ isotopes. Excited states in excess of $8 \mathrm{MeV}$ have been established resulting in the identification of $\square=4$, $15^{-}$and $13^{-}$isomers. Shell model calculations using modern interactions are able to reproduce spin, parity and excitation energies of these high-seniority states associated with complex configurations and thus providing confidence in extending these calculations towards doublymagic ${ }^{132} \mathrm{Sn}$ where the experimental data is more limited. 
- MNT reactions have been suggested as an alternative route to the formation of super-heavy elements. Programs to investigate this possibility have begun providing spectroscopic information on excited states for both even-even and odd- $A$ isotopes as heavy as $\mathrm{Cf}(\mathrm{Z}=98)$. These data are complementary to the fusion reactions which have been used to identify excited states in Fm $(\mathrm{Z}=100)$ through $\mathrm{Rf}(\mathrm{Z}=104)$ isotopes. These data help benchmark theoretical calculations with attempt to predict where the island of stability is located in the heaviest elements.

\subsubsection{Opportunities for the Near Future}

The sections below outline a few key programs per physics topic that will likely be addressed in the near future at existing facilities. These topics and programs are by no means complete and rather serve as examples of activities within our broad Nuclear Structure and Reactions community. Such an ambitious experimental program will require a significant amount of beam time at the present facilities. The needed facilities and the equipment for these programs largely exist or are in advanced stages of development and, with adequate support, will be ready in the short-term. Facilities and equipment that the various opportunities rely on are listed.

\subsubsection{At the Limits of Existence}

\section{Discovery of new isotopes:}

- Due to some of NSCL's world-unique primary beams, as for example ${ }^{76} \mathrm{Ge}$ and ${ }^{82} \mathrm{Se}$, cutting-edge isotope discovery opportunities continue to exist at the Coupled Cyclotron Facility prior to the FRIB area, in particular with targeted intensity and transmission upgrades and improved target technology.

Needed resources: fast beams at NSCL and in-flight separation

\section{Beyond the neutron drip line:}

- Identification of multiple neutron events offers the opportunity to study two-, three- or even fourneutron emitters. From the correlations it is possible to extract information about the internal structure of these nuclei. Multiple neutron emission will also be used to search for and identify alpha knockout as a new direct reaction process. The implementation of the active target will allow a precise lifetime measurement of the $2 \mathrm{n}$ radioactivity of ${ }^{26} \mathrm{O}$.

- $\beta$-delayed neutron spectroscopy will be used to map the $\beta$-decay strength distribution above the neutron separation energy, for fission fragments available from CARIBU at ATLAS and unique neutron-rich nuclei produced by projectile fragmentation at NSCL.

Needed resources: Fast beams at NSCL, MoNA-LISA/Sweeper@NSCL, LENDA, VANDLE, 3Hen, NERO, modular decay stations with r-ray detection, CARIBU@ANL and gas-stopping@NSCL

\section{Towards the heaviest elements:}

- Spectroscopy of transfermium nuclei at ANL and LBNL and test of their production through cold and hot fusion reactions will enable studies of nuclei around $\mathrm{Z}=100$ and $\mathrm{N}=152$ through in-beam spectroscopy, calorimetry and identification of $\mathrm{K}$ isomers and $\alpha$ fine structure.

- Synthesis of new neutron-rich heavy nuclei using multi-nucleon transfer reactions.

- Ongoing improvements in experimental capabilities will enable first $\mathrm{Z}$ and $\mathrm{A}$ identification of a superheavy element (with $\mathrm{Z}=114$ and higher).

- Chemistry experiments on long-lived isotopes of elements Rf, Db, Sg, Bh, Mt, Ds, Rg, Cn, 113, and Fl will be performed. The online chemistry program at TAMU has the opportunity to reestablish a US presence in the field of heavy-element chemistry. 
- Continuation of strong US involvement in the production of new elements at international facilities, afforded by rare actinide targets produced in the US.

Needed resources: BGS@LBNL,FMA/AGFA@ANL,DGS/GRETINA@FMA/AGFA, HIFR@ORNL, digital DAQs, online chemistry facility at TAMU accelerator

\subsubsection{Evolution of Nuclear Structure}

- Transfer reactions, enabled by HELIOS or $\gamma$-ray tagged with charged-particle detector arrays such as GODDESS, will be used with the newly available ReA3 and CARIBU beams for the exploration of single particle structure across the regions of the nuclear chart opened up by fission fragment beams and reaccelerated beams of rare isotopes produced by projectile fragmentation.

- Proton transfer with neutron detector systems will be developed into a standard technique, similar to (d,p) in inverse kinematics. Time-of-flight and liquid scintillator detectors have been developed for use in single-proton transfer reactions such as $(\mathrm{d}, \mathrm{n})$.

- Afforded by the unique combination of world-class experimental equipment, GRETINA (including GRETINA enhancements) coupled to the S800 spectrograph and using the TRIPLEX plunger for excited-state lifetime measurements, in-beam $\gamma$-ray spectroscopy in pursuit of tracking the evolution of nuclear structure (knockout, pickup, Coulomb excitation, secondary fragmentation, CEX) will increase the reach of these experiments closer to the neutron dripline, to isotopes with one or two more neutrons than possible now, opening up cutting edge opportunities at the extremes of the nuclear chart with fast-beam experiments at NSCL.

- Decay spectroscopy with modular detection systems and increased sensitivity due to advanced digital signal processing will exploit unique opportunities for the study of fission fragments from CARIBU at ANL and very neutron-rich and neutron-deficient nuclei produced by projectile fragmentation at NSCL. The detection of multiple radioactivities in a decay process affords, for example, novel studies of shape coexistence through conversion electron spectroscopy.

- $100 \mathrm{MeV} / \mathrm{u}$ beams and neutron- and $\gamma$-ray spectroscopy in coincidence with high-resolution particle detection with the S800 spectrograph will allow the unique tracking of changes in the nuclear structure through measurements of weak-interaction strengths from charge-exchange reactions.

- The broad range of reaccelerated rare isotope beams at ReA3 and the unique fission fragment beams available at CARIBU will allow for measurements of transition matrix elements, excited-state quadrupole moments and $\mathbf{g}$ factors in regions of shell evolution around neutronrich $\mathrm{S}$ and Ar nuclei approaching $\mathrm{N}=28$, along the $\mathrm{Ca}$ and $\mathrm{Ni}$ isotopic chains and in the broader region around ${ }^{132} \mathrm{Sn}$, respectively.

- Multi-nucleon transfer reactions induced by stable beams at ATLAS/ANL combined with coincident $\gamma$-ray spectroscopy with digital Gammasphere or GRETINA/GRETA will allow exploring the changes in the nuclear structure at high spin and allow tracking the decent of intruder states from stability toward (new) regions of shell evolution.

Needed resources: fast beams at NSCL, S800@NSCL, TRIPLEX/GRETINA@NSCL, ReA3@NSCL, CARIBU@ANL,ATLAS@ANL, GRETINA@NSCL/ANL, existing r-ray detector arrays at NSCL+ANL, HELIOS@ANL, modular decay stations, both Si- and CsI(Tl)- based light-charged particle arrays at ANL+NSCL,CHICO@ANL/NSCL, LENDA/VANDLE@S800

\subsubsection{Collective Nuclear Degrees of Freedom and Simple Patterns in Complex Nuclei}

- With beams from CARIBU at ANL, scarcely explored regions of triaxiality and octupole collectivity will be available for in-beam experiments, for the first time, using for example Coulomb excitation for the extraction of the quadrupole and octupole transition matrix elements and static moments. 
- The enhanced data-rate performance of Gammasphere at ANL will be used to continue the study of new phases of collectivity at ultra-high spins approaching $\mathrm{I}=70 \hbar$.

- While much has been done to quantify the nuclear structure at superdeformed shapes, no evidence for excited states in the theoretically predicted third-minimum have been observed. The deformation of these states are even higher than that for superdeformed nuclei and would provide another laboratory to study nuclei in the vicinity of large shell gaps. With the increased resolving power of digital Gammasphere and GRETINA/GRETA, the identification of states associated with these extremely deformed nuclei may be possible.

Needed resources: fast beams at NSCL, S800@NSCL, TRIPLEX/GRETINA@NSCL, ReA3@NSCL, CARIBU@ANL,ATLAS@ANL,CHICO@ANL/NSCL, GRETINA@NSCL/ANL, existing $\gamma$-ray detector arrays at NSCL+ANL

\subsubsection{Precision Measurements of Ground-State Properties}

\section{Masses, radii, and moments}

- Mass spectrometry at CARIBU/ANL and at the stopped-beam facility at NSCL will allow for world-unique measurements of ${ }^{252} \mathrm{Cf}$ fission fragments and rare isotopes produced by projectile fragmentation, respectively. Upgrades of existing facilities to single-ion capabilities and multireflection time-of-flight spectrometers will increase the sensitivity and the reach at the existing facilities. New mass measurement programs at TAMU and Notre Dame will complement the programs at the user facilities.

- Unique and complementary laser spectroscopy programs are starting up at CARIBU/ANL and with BECOLA at NSCL. For example, pioneering collinear laser spectroscopy of Pd, Sb, Rh, and $\mathrm{Ru}$ fission fragments and in-flight produced ${ }^{8} \mathrm{~B}$ will be possible at ANL and world-unique light transition metals (V-Ni) will be available for measurements at NSCL's BECOLA facility.

- Magnetic moments and quadrupole moments of unique transition metals, for example, will be measured with optical pumping combined with $\beta$-NMR and $\beta$-NQR techniques at BECOLA.

Needed resources: Gas-stopping@NSCL,CARIBU@ANL,BECOLA@NSCL,LEBIT@NSCL,CPT@ANL and laser-spectroscopy@CARIBU

\subsubsection{Correlations in Nuclei}

- With sensitive detection techniques (e.g., TPCs), opportunities exist for studying $2 p$ correlations up into the fpg shell.

- Along isotope chains, pairing in a variety of density and nuclear binding regimes can be probed via low-energy direct $2 n$ or $2 p$ transfer reactions, in light nuclei from the drip lines toward stability.

Needed resources: ReA@NSCL,CARIBU@ANL, AIRIS/ATLAS@ANL, HELIOS, Si-based arrays such as HIRA, ORRUBA, SuperORRUBA, TUDA, or TECSA, active target detectors and TPCs, GRETINA, DGS and other existing $\gamma$-ray detection arrays, particle ID detectors

\subsubsection{Studies of Reaction Mechanisms}

Many important nuclear-structure results rely on reaction theory for their interpretation. Much of the theory applied to nuclear reactions dates from the 70's, and was validated with data from reactions on nuclei near stability. Effects such as breakup, coupling to the continuum, and resonant-non-resonant interference are not well understood and comprehensive data with well-understood nuclei are necessary before modern nuclear-reaction theories can be trusted far from stability. One major goal is to establish a 
microscopic, predictive basis for constructing optical-model potentials, rather than deriving them empirically from scattering data that may not be available for very exotic nuclei.

- High-intensity stable beams inducing multi-nucleon transfer reactions on heavy targets will be used at ATLAS/ANL and TAMU to explore promising predictions and initial experimental evidence on the viability of multi-nucleon transfer to produce heavy neutron-rich nuclei.

- At ReA at NSCL, unique beams produced by fragmentation will be used, for the first time, for fusion and fission studies. The role of neutron excess can be explored, for example, with fusion induced by neutron-rich and neutron-deficient Ar and S beams.

- Guidance is needed from experiment in the form of detailed high-quality scattering data with very neutron-rich beams, and also for reactions involving complex light ions such as ${ }^{3} \mathrm{H},{ }^{3} \mathrm{He},{ }^{4} \mathrm{He},{ }^{6} \mathrm{Li}$, etc., where current reaction theory is far behind modern developments for nucleon-stripping reactions.

- Extrapolations of our understanding of optical-model potentials, derived from reactions on nuclei near stability, are proving unreliable when taken to very neutron-rich or proton-rich nuclei. Measurements of multiple observables under carefully controlled conditions are needed to validate the predictions of reaction theories, before they can be reliably applied to reactions with very exotic nuclei.

Needed resources: ReA@NSCL,CARIBU@ANL, AIRIS/ATLAS@ANL,T-REX@TAMU, HELIOS,Sibased arrays such as HIRA, ORRUBA, SuperORRUBA, TUDA, or TECSA, GRETINA + other existing $\gamma$ ray detection arrays, particle ID detectors

\subsubsection{Long-Term Opportunities}

The unprecedented reach of Facility for Rare Isotope Beams (FRIB) will provide access to unexplored regions of the nuclear chart, where new phenomena like large nucleon skins and new collective modes will guide the development of a more comprehensive picture of the atomic nucleus, likely changing known paradigms on the structure of atomic nuclei. With the neutron drip line in reach with FRIB up to approximately $\mathrm{A}=120$, the field will have the opportunity to produce $80 \%$ of all isotopes for the elements below uranium. From this vast pool of nuclei, key isotopes can be chosen for individual experiments because they amplify or isolate a specific physics question.

At FRIB, key isotopic chains with multiple neutron magic numbers, for example $\mathrm{Ca}$ and $\mathrm{Ni}$, will come into reach from drip line to drip line, allowing to constrain the isosopin dependence of the nuclear force and continuum effects broadly. This is required to constrain the effective many-body Hamiltonian. Nuclei with large halos and extended neutron skins will become available and revolutionize the field of nuclear reactions.

The program at FRIB will be complemented with cutting-edge research programs at ANL requiring highintensity stable beams to explore nuclei under extreme conditions. Programs at university laboratories will remain critical to efficiently target specific programs with stable and selected rare-isotope beams, in addition to providing opportunities for equipment developments and hands-on education of young researchers. 


\subsubsection{At the Intersection to Hadronic Physics - Near-Term and Long-Term Opportunities for Nuclear Structure Research at Jefferson Lab}

- Electron-induced nucleon knockout: The ability to determine the detailed structure of nuclei has turned out to be invaluable to the neutrino community which uses nuclear targets to extract information about neutrino-nucleon interactions. In particular, future Jefferson $\operatorname{Lab}{ }^{40} \operatorname{Ar}(e, e ' p)$ measurements will be used to provide input to neutrino community's Monte Carlo event generators. In addition, D(e,e'p)n measurements which will make use of $11 \mathrm{GeV}$ electrons will push our understanding of the nucleon-nucleon potential by making knock-out measurements in extreme kinematic conditions while the ${ }^{3} \mathrm{H}(\mathrm{e}, \mathrm{e}, \mathrm{p}) /{ }^{3} \mathrm{He}(\mathrm{e}, \mathrm{e}$ 'p) measurements will allow for separation of proton and neutron distributions in $\mathrm{A}=3$ nuclei. Looking to the long term, an Electron-Ion Collider would enable measurements of nucleon knock-out reactions in channels that are not accessible in (e,e'p) fixed-target experiments and may offer unique opportunities for novel studies of low-energy nuclear structure.

- EMC effect: With the completion of the Jefferson Lab $12 \mathrm{GeV}$ upgrade, several new experiments are planned to precisely map out the slope of the EMC effect as well as the magnitude of the short-range correlations in many nuclei to finally solve the EMC effect puzzle. In addition, tagged measurements on a nucleon in ${ }^{2} \mathrm{H}$ will allow for separating free nucleon structure from nuclear effects associated with highly off-shell nucleons and/or small-sized components of the deuteron.

- Hypernuclei: Future hypernuclear experiments on light hypernuclei will focus on investigating the nucleon $\Lambda$ interaction including studying charge-symmetry breaking effects as well as the nucleon $\Lambda$ and nucleon $\Sigma$ couplings. Precision spectroscopy of heavy hypernuclei will examine the effectiveness of mean-field theory and shell-models and explore the effects of three- and fourbody forces in the baryonic many body systems. These measurements can also help understand the role of strangeness in the equation of state for baryonic many-body systems.

- Neutron skins: With the success of the pioneering measurement on lead, new parity violation measurements are now planned for the neutron rich ${ }^{48} \mathrm{Ca}$ and ${ }^{208} \mathrm{~Pb}$ nuclei with planned precisions of $0.02 \mathrm{fm}$ and $0.06 \mathrm{fm}$ respectively. The results from these measurements will be interesting not only from a nuclear structure point of view, but also for nuclear astrophysics where the results will be used to better our understanding of the equation of state for nuclear matter and thus improve our understanding of neutron stars.

\subsubsection{Necessary Resources}

The Nuclear Structure and Reaction community needs the opportunities provided by FRIB to address the challenges of the field that were consistently articulated by the community and that were most recently summarized and reaffirmed in the National Academy Decadal Study (2012). The unmatched beam intensities that will be provided by FRIB over a range of energies from thermalized to $200 \mathrm{MeV} / \mathrm{u}$ affords unique opportunities to understand and model atomic nuclei and learn how these many-body systems interact in nuclear reactions.

In preparation for FRIB, targeted equipment developments are needed to realize the day-1 science opportunities readily. Much of the needed equipment is being developed and used at the existing facilities as FRIB is under construction, providing the opportunity to have commissioned equipment ready to run when FRIB turns on while producing cutting-edge science at existing facilities prior to the start of FRIB.

Many science opportunities that involve direct or compound nuclear reactions rely on the availability of reaccelerated rare-isotope beams with energies exceeding $10 \mathrm{MeV} / \mathrm{u}$ across all species. The availability of these rare-isotope beams produced in chemistry-independent fragmentation reactions will be world unique and a game changer for the nuclear structure and reactions community. 
World-class rare-isotope and stable-beam programs producing cutting-edge nuclear structure and reactions science are ongoing at the premier US user facilities NSCL and ANL. Resources are needed to keep both facilities and their equipment at the cutting edge on the path into the era where FRIB and ANL, respectively, will be the premier rare-isotope and stable-beam facilities in the world with a prepared user community ready for science. Vibrant nuclear structure and reactions programs are being carried out at the university-based laboratories where targeted developments and setups provide unique opportunities these efforts are complementary and crucial in preparing the community for FRIB and need to be maintained.

The study of nuclear structure and reactions is extremely broad and employs a wealth of complementary experimental techniques that address research areas ranging from precision ground state properties to the study of shell evolution or the quest for the heaviest elements. Many areas use the direct reactions to study the structure of nuclei. Direct reactions are applied across the entire range of energies ranging from a few $\mathrm{MeV} / \mathrm{u}$ to several hundred $\mathrm{MeV} / \mathrm{u}$. At the lower beam energies, particle-ID detectors or recoil separators are needed to identify the projectile-like reaction residue following transfer-type reactions. Light-particle detection is typically accomplished with Si-based detection systems for charged particles and neutron detectors for transfers with neutrons in the exit channel. Gamma-ray tagging is used more and more to increase the resolution as well as open opportunities to measure excited-state lifetimes, $\gamma$ particle correlations etc., and GRETA will be a game changer there. New concepts, like the HELIOS spectrometer or the GODDESS system, push the resolution frontier in transfer reactions. Investments are needed to keep this smaller-scale equipment and the detection systems at the cutting edge. Once ReA12 exists at NSCL or FRIB, a large-acceptance recoil separator would optimize the use of direct reactions with reaccelerated beams. For research programs with fast beams, which provide the furthest reach into uncharted territory, the S800 spectrograph provides large acceptance and high resolution at the present NSCL facility. In the FRIB era, however, the High-rigidity Spectrometer (HRS) is needed to fully exploit the opportunities with the most exotic beams which are efficiently produced at rigidities beyond the capabilities of the S800.

In-beam spectroscopy, which often but not exclusively uses direct reactions, relies on $\gamma$-ray detection for the exploration of bound states and on neutron detector arrays for the detection of neutron-unbound states. The in-beam $\gamma$-ray spectroscopy community, for measurements with stable beams as well as fast and reaccelerated rere-isotope peams, rallies around GRETA, which will provide unprecedented efficiency and resolving power for in beam measurements at NSCL and ANL now and FRIB in the future. Invariant mass spectroscppy beyond the neutron drip line requires the HRS in the FRIB era, as NSCL's Sweeper Magnet is too limited in magnetic rigidity and acceptance while the neutron array MoNA-LISA is ready for FRIB.

Active decay spectroscopy programs are in place at NSCL and ANL that need to be kept at the cutting edge in addition to the pow werful detection systems are maintained by the ORNL and UT groups. In the long-term, the decay spect oscopy community is developing a new decay station based on the modular detection of all possible radjations following a decay.

Precision measurements of ground-state properties rely on laser spectroscopy and trap-assisted mass spectrometry with broad programs in place at ANL and NSCL. These setups are largely ready for the next stages of these facilities with improvements and developments ongoing. Multi-reflection TOF (MR-TOF) spectrometers for mass measurements of the shortest lived species are already under development as well. Equipment is in place to study g-factors and Q-moments in-beam with reaccelerated rare isotopes. Efficient $4 \pi \gamma$ ray detection systems, for example GRETA, are needed. 
In the quest to study the heaviest elements, AGFA and the BGS afford near-term opportunities in the US with target developments planned at LBNL. Charged-particle arrays and efficient $\gamma$-ray detection have to be kept at the cutting edge. Continued support is required for the US involvement in the unique superheavy element searches in Dubna and RIKEN. A main, but not the only, driver for the exploration of reaction dynamics is the goal to devise reaction schemes to produce the heaviest nuclei. Fusion and fission studies that account for the formation and survival probabilities of heavy compound nuclei rely on stable beams and reaccelerated rare-isotope beams and often Si-based charged-particle detectors and possibly neutron detectors. At ReA12, a recoil separator would allow the study of multi-nucleon transfer reactions induced by rare-isotope beams as well as fusion and fission. For these studies, final-state identification through in-beam $\gamma$-ray spectroscopy would be made possible with GRETA.

Much of the progress in the understanding of nuclei has come from experiment and theory working in concert and support for the research portfolio in experiment and theory is needed to continue enabling the ongoing cutting-edge nuclear structure and reactions research.

\subsubsection{Scientific Impact on Other Fields and Interdisciplinary Connections}

The connections that have evolved over the years between basic nuclear science and other basic and applied research fields remain strong. With the increased reach of FRIB into previously uncharted regions, the opportunities for applied and interdisciplinary research will grow. A wide variety of different scientific and technical areas rely on information obtained from low-energy nuclear science. For example:

Astrophysics: Cataclysmic events in the cosmos are driven by complex networks of nuclear reactions, and one of the most important questions, highlighted by the National Academy Decadal Study (2012) is understanding the formation of the elements heavier than iron. Low-energy nuclear-structure studies have and will continue to reveal states important to the production of the rare isotopes involved in violent astrophysical events. Studies of neutron-transfer on neutron-rich nuclei above the nickel region and stretching beyond lead need to be understood to reduce the nuclear physics uncertainties in r-process nucleosynthesis.

Fundamental interactions: Nuclear structure information constitutes critical input to fundamental symmetries studies, such as understanding parent and daughter isotopes used in neutrino-less double $\beta$ decay studies and the constraints on the nuclear matrix elements involved in this rare process that may reveal the neutrino mass hierarchy. Research carried out at low-energy facilities has led to new results on $\beta$-neutrino correlations, and the most sensitive determinations of super-allowed $\beta$-decay transitions that inform the current understanding of the CKM quark-mixing matrix emerge from low-energy nuclear science. In the past few decades, it has been realized that octupole deformation and enhanced octupole vibrations can strongly amplify the sensitivity of a permanent electric dipole moment (EDM) in atoms to the underlying physics beyond the standard model - the identification and quantification of octupole collectivity has been a prominent topic in nuclear structure research.

Engineering and materials (nuclear power): The nuclear-power industry relies heavily on data measured and techniques developed at low-energy nuclear-science facilities. The disposition of long-lived fission fragments produced as waste in reactors is a continuing problem. Understanding of the $\beta$-decay properties of these fragments, using instruments such as the Modular Total Absorption Spectrometer (MTAS) will be applied to the study of the decay heat from neutron-rich isotopes. Such information is crucial to the prediction of the performance of alternate nuclear fuels after an emergency shutdown.

Security (stockpile stewardship and homeland security): Nuclear science is central to many nationalsecurity issues. Data for nuclei away from stability obtained from reaction studies that are surrogates for neutron capture or neutron-induced fission continue to provide information necessary to understand the 
performance and state of the national nuclear stockpile ("Stockpile Stewardship"). Some of the nuclei important for the complex reaction networks will only be available for surrogate reaction studies at FRIB. Radiography and optimized detection techniques are widely used to monitor and detect illicit materials and the needs from nuclear science for improved methods will only grow in the coming years. For these topics, neutron and gamma induced reactions play prominent roles, and the resulting complex reaction networks are difficult to calculate using modern reaction theory.

Medicine: Imaging techniques that utilize radio-isotopes are widely used in medicine today. Detectors used in medical imaging have evolved from basic-research instrumentation used in low-energy nuclearphysics research. Radioactive isotopes, such as ${ }^{99} \mathrm{Tc}^{\mathrm{m}}$, are central to a huge array of diagnostic imaging techniques currently in use, such as Positron-Emission-Tomography (PET) and PET-Computed Tomography (PET-CT). Radiation has long been used for cancer treatment, and modern approaches such as proton- and heavy-ion-beam radio-therapy are becoming available as more targeted approaches for the destruction of tumors. Research and development work on isotope production, mostly for medical applications continue, and new partnerships with the isotopes industry, for example at the University of Notre Dame, are developing.

Devices: The ubiquitous presence of semiconductor devices in all facets of modern life makes us sensitive to problems that many people are not aware of. These devices can be affected, for instance, by the radiation in the environment around us. Critical integrated circuits in the power grid, aircraft, and satellites can be disturbed or even damaged by cosmic radiation. These effects can, however, be studied by exposing those devices to beams of ionizing particles produced in the laboratory. Information from such measurements can guide design principles for integrated circuits that can significantly reduce their sensitivity to radiation. 


\subsection{Nature of Dilute and Dense Nuclear Matter and Equation of State}

\subsubsection{Introduction}

One of the fundamental goals in nuclear physics is to understand the nature and properties of dilute and dense nuclear matter. The bulk properties of nuclear matter are described by the nuclear Equation of State (EOS) which not only determines many properties of finite nuclei but also controls the form and life cycle of astrophysical objects. This dual focus of the study of the EOS is best illustrated by a few examples below. More detailed discussion of these and other issues can be found in later sections.

- The symmetry energy at high densities, if it can be constrained by heavy-ion collisions, would be the laboratory constraint that is most directly relevant to the structure of neutron stars and the phases of cold dense QCD. Theoretical predictions suggest new measurements to investigate the symmetry energy at supra-normal densities using heavy-ion collisions.

- The symmetry energy at finite temperature and low density does not go to zero - as it would for a gas of free nucleons. This reflects correlations and cluster formation in the low-density medium. Clustering also affects the surface properties of finite nuclei and thus the low-energy reactions between nuclei.

- The predicted strong correlation between the neutron-skin thickness and the first derivative of the symmetry energy with density, L, which is related to the pressure of pure neutron matter, allows probing the structure of neutron stars by studying the structure of nuclei on earth.

- Dispersive Optical Model (DOM) analyses using massive data sets at both positive (unbound scattering) and negative (bound) energies to fix real and imaginary components of nuclear potentials, inform on spectroscopic factors and the potentials needed for scattering theory but also provide insight into the symmetry potential and effective nucleon masses.

- Studies of the asymmetry dependence of nuclear incompressibility using the giant monopole resonance reveal a conundrum for nuclear structure - Sn isotopes, both neutron-rich and neutrondeficient, are more compressible than predicted.

The Equation of State for symmetric matter has been determined from ground-state properties of nuclei, from isoscalar collective vibrations near saturation density, and at higher density, from collective flow and kaon production. Since the last long-range-plan, there has been significant progress in experimentally determining the symmetry energy at near and below the saturation density and in observation of the nonzero, and nearly constant, symmetry energy at very low density. However, at densities greater than saturation, $\rho_{o} \sim 0.15 \mathrm{fm}^{-3}$, the symmetry term is largely unconstrained. The challenge in the next decade and during the next long-range-plan period is to extend our knowledge on the EOS to neutron-rich and ultimately to pure-neutron matter. This requires the determination of the density dependence of the asymmetry term in the EOS. This knowledge is essential to our understanding of nuclear masses and sizes, the existence and location of the neutron-drip line, the dynamics of nuclear collisions, and the properties of neutron stars, supernovae and black hole accretion disks.

\subsubsection{Compelling Scientific Opportunities: Near-Term and Long-Term}

Creation of both dilute and dense neutron-rich nuclear matter in the laboratory environment requires a diverse range of reaction probes. This subfield of nuclear science encompasses experiments, analyses and the development of nuclear models that connect the structure of, and the reactions between, finite nuclei to the properties of bulk nuclear matter. Through variation of reaction mechanism, beam energy, and 
impact parameter one can "tune" observables to specific density regions. In the following, we discuss the physics opportunities from low to above saturation density regions.

\subsubsection{Recreating Neutrinosphere Conditions on Earth}

Interestingly, conditions similar to the neutrinosphere (the locus of last neutrino scattering) in supernovae, i.e., very low density, asymmetric matter with temperatures of about $5 \mathrm{MeV}$, can be created in intermediate energy heavy-ion reactions. Correlations and cluster formation in neutrinosphere matter (at fixed overall density) modify the neutrino and antineutrino opacities. Experimental investigations of the asymmetry dependence of cluster formation, combined with more sophisticated theoretical formulations are needed to constrain the models for the corresponding astrophysical equations of state, the composition of neutrinosphere matter, and to understand the resulting neutrino interactions.

In the neutron-rich medium near the supernova neutrinosphere, the absorption of an electron neutrino, $v+n \rightarrow p$
the produced proton is morts a neutron into a proton and the symmetry energy is released. This is because phase space and the cross section. Fig. 1 shows theoretical results for neutrino absorption cross sections under different assumptions. The black dot-dashed lines are for a calculation that ignores all effects of the symmetry energy, while the calculation represented by the red-dashed lines includes a small symmetry energy that is proportional to the (low) density. Finally the blue-shaded region indicates the consequences of a large symmetry energy that includes correlation effects.

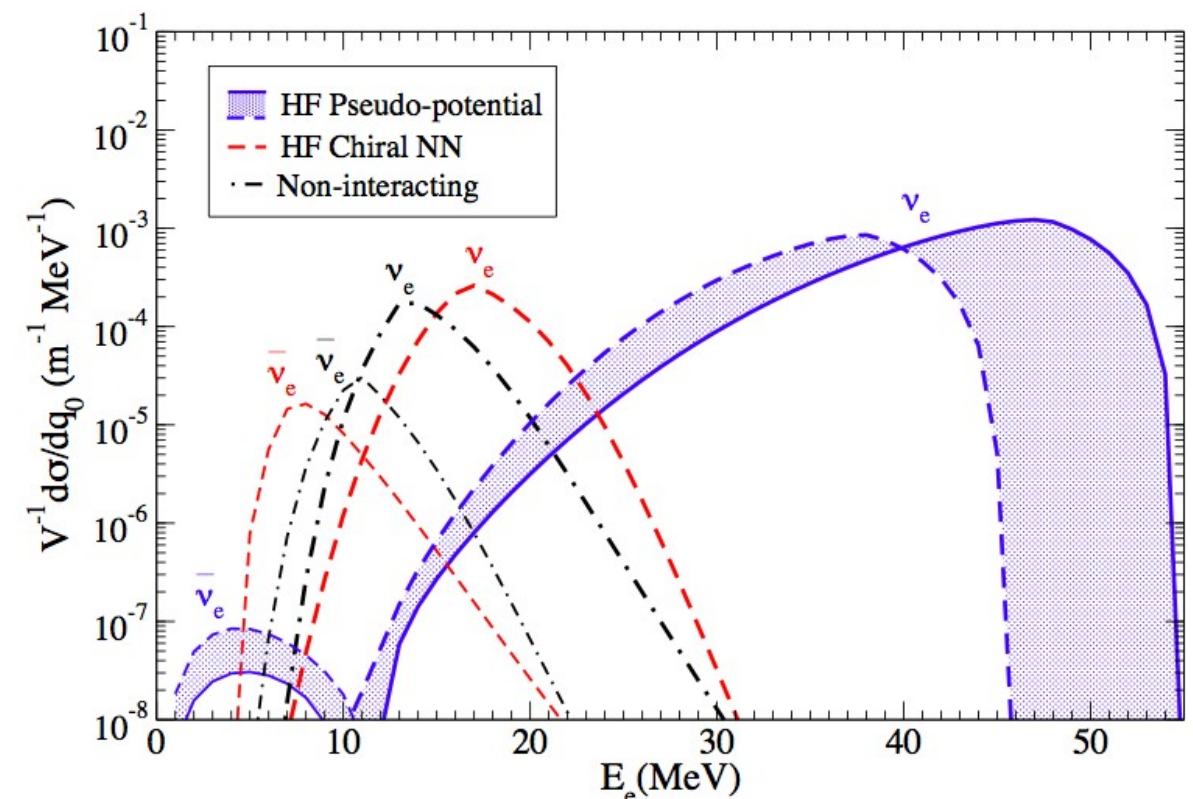

Figure 1: Cross section per unit volume for neutrino absorption versus the energy of the outgoing charged lepton. This is for an incoming neutrino energy of $12 \mathrm{MeV}$, a density of 0.02 per cubic fm and a temperature of $8 \mathrm{MeV}$, see text [Figure from E. Rrapaj et al., arXiv:1408.3368].

A large increase in the neutrino absorption cross section will keep neutrinos in thermal equilibrium with the supernova matter to lower densities and temperatures. As a result the emitted neutrino spectra will be softer (lower energy). During a supernova explosion, the intense neutrino fluxes drive some baryons off of the newly formed neutron star. This change in spectrum will make the neutrino driven wind more neutron rich and change the resulting nucleosynthesis. 


\subsubsection{Impact of Clustering at Very Low Density and in Nuclear Surfaces}

Experimental evidence to date suggests that at temperatures around $5 \mathrm{MeV}$, the symmetry energy remains near $10 \mathrm{MeV}$ due to clustering. Such clustering also has an impact on nuclear structure. For example, based on the compatibility of single-particle valence orbits, one might expect light Sn isotopes to have a substantial alpha clustering in their surfaces while highly asymmetric $\mathrm{Sn}$ isotopes would have little. Experimental investigations of alpha cluster formation on the (low-density) surfaces of nuclei as a function of the neutron number of isotopes will provide information about the alpha "preformation factor" as a function of asymmetry, which plays a role in alpha emission and cluster transfer in peripheral reactions at low energy.

\subsubsection{Astrophysical Relevant Fission and Fusion Measurements}

Both fission and fusion studies at low energy are sensitive to the tail of the nuclear matter distributions. Furthermore the fissility of a neutron-rich nucleus far from stability depends on the surface asymmetry which is dictated by the symmetry energy at low density. Pycnonuclear (density driven) fusion of very neutron-rich nuclei near the drip line may be an important source of deep crustal heating in accreting neutron stars. Measurements of sub-barrier fusion cross sections with beams like ${ }^{20} \mathrm{O}$ will constrain the effects of very neutron-rich skins on astrophysically relevant fusion reactions and allow searches for new fusion dynamics associated with very polariazable skins.

\subsubsection{Neutron Skins in Heavy Nuclei}

Assuming all the neutrons and protons are distributed separately in a ${ }^{208} \mathrm{~Pb}$ nucleus, calculations show that the neutron radius $\left(r_{n}\right)$ is larger than the proton radius $\left(r_{p}\right)$. A number of models show strong correlations between the neutron-skin thickness, $\Delta \mathrm{r}_{\mathrm{np}}$, and the first derivative of the symmetry energy with respect to density, L (i.e. slope of the Taylor expansion of the symmetry energy). $\mathrm{L}$ is related to the pressure of pure neutron matter. An example of the calculated correlation from representative models is shown in Fig. 2.

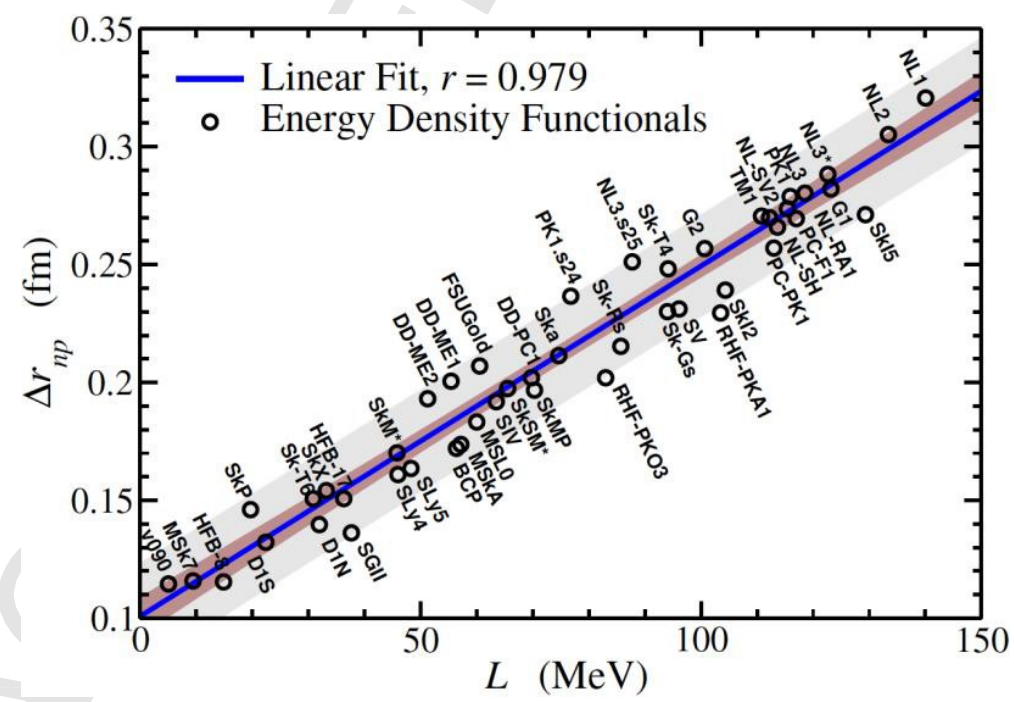

Figure 2: Correlation of the neutron-skin thickness (difference in neutron and proton root-mean-square radii) of ${ }^{208} \mathrm{~Pb}$ as a function of the slope of the symmetry energy, L, predicted by a representative group of Density Functional Models. The thicker shaded region represents the 95\% prediction band of the linear regression. This figure is adapted from Ref. [X. Roca-Maza, M. Centelles, X. Vinas, and M. Warda, Phys. Rev. Lett. 106, 252501 (2011)] 
The strong correlations between $\Delta \mathrm{r}_{\mathrm{np}}$ and L motivate many measurements of the neutron-skin thickness of ${ }^{208} \mathrm{~Pb}$ nucleus. However, analysis of measurements with hadronic probes often suffer from large model uncertainties. The first measurement of the neutron-skin thickness ${ }^{208} \mathrm{~Pb}$ using parity violating electron scattering (PREX) with a clean theoretical interpretation with small systematic errors was carried out in 2010. The present result, $\Delta \mathrm{r}_{\mathrm{np}}=0.33+0.16-0.18 \mathrm{fm}$, with its large statistical uncertainty cannot exclude most theories. It is important that follow up experiments (PREXII) with greatly improved statistics are run in the next long-range-plan period.

In addition to sensitivity to the symmetry energy, systematic data on neutron radii provides constraints on the nuclear energy density functional in the prediction on the location of the neutron drip line, and shell structure. Electron probes to measure skin thickness at JLab can only be applied to a few nuclei as these experiments are difficult. Hadronic probes are being developed to provide reliable measurements of the skin thicknesses over a wide range of isotopes. Ideally, measurement of the skin thickness of the same nuclei such as ${ }^{208} \mathrm{~Pb}$ by both the electron and hadron probes, allows cross comparisons between the two different probes and facilitates development of better models used in the hadron probe analysis.

\subsubsection{Density Dependence of Symmetry Energy Around Saturation Density}

Significant progress has been made in understanding the density dependence of symmetry energy, at below, and near the saturation densities. The current symmetry energy constraints from heavy-ion collisions using isospin diffusion and neutron and proton spectra are obtained mainly from stable beam experiments. Expanding such measurements using radioactive beams increases the asymmetry of the collision systems and thus the sensitivities to symmetry energy. New measurements with radioactive beams and use of new observables such as the time dependence of the isospin diffusion, should improve the current uncertainties of the constraints.

\subsubsection{Density Dependence of Symmetry Energy Above Saturation Density}

Laboratory constraint on the symmetry energy at high densities, is most directly relevant to the structure of neutron stars. In the next decade, exciting opportunities lie in the exploration of symmetry energy at densities around twice the saturation density which strongly affects neutron star radii. Currently there is no consensus as to the behavior of the symmetry energy above saturation density. A recent, and controversial, analysis of quiescent low-mass x-ray binaries (qLMXB) in globular clusters yields a very small radius of neutron stars, $9.1+1.3-1.5 \mathrm{~km}$. This suggests a softening of the symmetry energy at density around twice the saturation density. However such a softening would have to be followed by a stiffening in order to explain neutron stars with masses exceeding twice the solar mass, of which there are now two cases. Nuclear matter above saturation density can only be explored in the laboratory with heavy-ion collisions. New observables and new detectors will be needed to meet this challenge.

\subsubsection{Major Accomplishments Since the Last Long Range Plan:}

Since the last Long Range Plan, experiments and analyses have been undertaken to extract temperature, pressure, energies and free energies in the relevant thermodynamic state space $\left[T, \rho=\rho_{n}+\rho_{p}, \delta=\left(\rho_{n}\right.\right.$ $\left.\rho_{\mathrm{p}}\right) / \rho$ ]. The range of accessible asymmetry $\delta$ has been expanded with availability of secondary beams at many facilities around the world and will be greatly expanded when FRIB becomes operational.

At densities $\rho \sim 0.1 \mathrm{fm}^{-3}$, Skyrme interactions are highly constrained by the average nuclear properties. Thus the symmetry energy, should be reasonably well known. Employing the ground-state properties of double magic nuclei plus the masses of 38 spherical nuclei, symmetry energy values of $25.4 \pm 0.8 \mathrm{MeV}$ at 
$\rho=0.10 \mathrm{fm}^{-3}$ and $26.65 \pm 0.2 \mathrm{MeV}$ at $\rho=0.11 \mathrm{fm}^{-3}$ were obtained. These are represented by the red circle and red square plotted in Figure 3. One can use ab-initio calculations to determine the shape of the EOS. However, extrapolations to substantially higher or lower densities using these two points are highly uncertain due to the small lever arm, i.e. small and uncertain difference in the abscissa values.

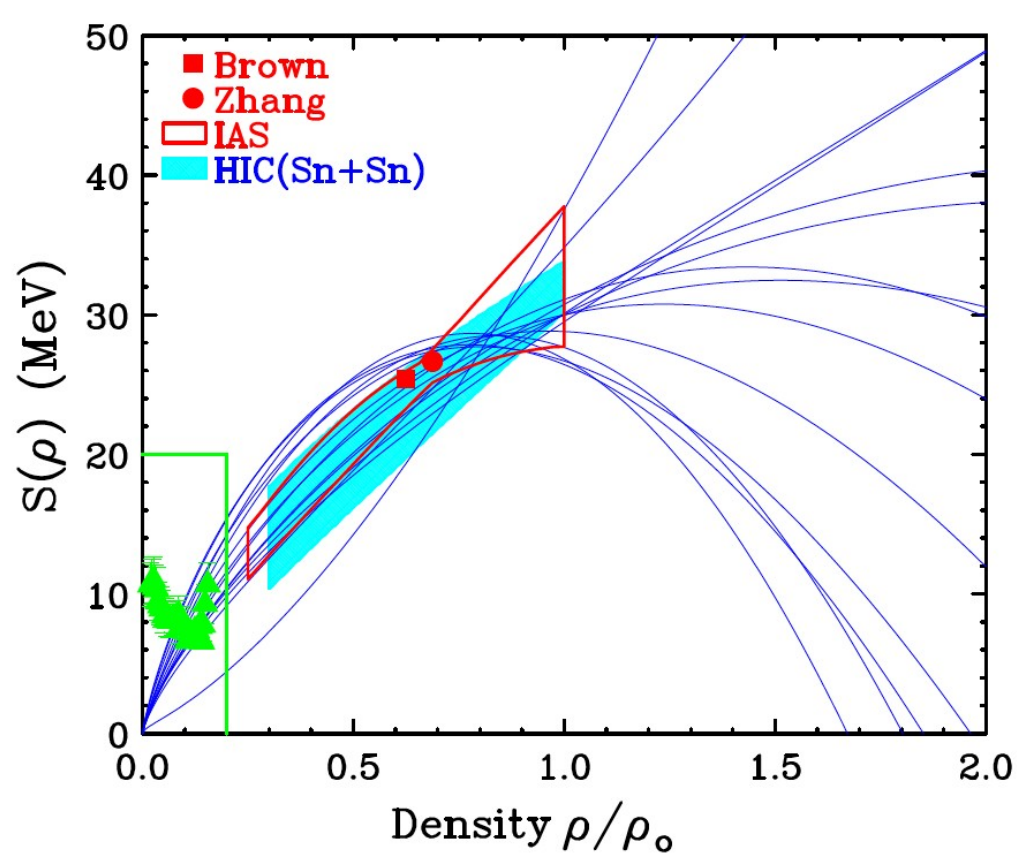

Figure 3: Constraints on the density dependence of the symmetry energy. The lines are predictions from common Skyrme interactions showing the divergence of the symmetry energy dependence above saturation density. The two red symbols are obtained by fitting density functionals with selected Skyrme interactions to masses (see text for details). The polygonal box is obtained from analyzing the masses of the Isospin Analog States. The light blue shaded area labeled HIC $(S n+S n)$ is obtained using isospin diffusion observables constructed from heavy-ion collision data of ${ }^{112} \mathrm{Sn}$ and ${ }^{124} \mathrm{Sn}$ in identical and mixed combinations at $50 \mathrm{MeV} /$ nucleon. The green data points on the left part of the figure are obtained from the analysis of cluster production in heavy ion collisions. It should be noted that these represent the global symmetry energy including cluster correlations in non-uniform matter at a temperature of several MeV, depending on the density. (Adapted from Ref. [C. Horowitz et al., J. Phys. G 41, 093001 (2014)].

Since the last Long-Range Plan a number of experimental techniques have been developed to probe properties of the neutron skin of medium- and heavy-mass nuclei. These include proton elastic scattering for precise determination of the GDR strength distribution in nuclei with large neutron-proton radius asymmetries and the associated determination of the electric dipole polarizability and studies of the Pygmy Dipole Resonance (PDR). Also, providing new insight are gamma-ray beam based techniques, e.g., nuclear resonance fluorescence to study the PDR and Compton scattering for precise characterization of the Isovector Giant Quadrupole Resonance and determination of the electric dipole polarizability of nuclei. Of particular importance is the theoretical development that shows a tight correlation between the polarizability, $\alpha$, and the product of the symmetry energy at saturation (J) and (slope) L. Success of these measurements paves the way to further measurements on the skin thickness of a wide range of nuclei.

Figure 4 provides another illustration of how EOS issues are entwined with nuclear structure. This figure displays the recently appreciated dependence of $\Delta \mathrm{r}_{\mathrm{np}}$ in ${ }^{208} \mathrm{~Pb}$ (and thus L, see Fig. 2) on three body neutron forces as extracted from hadronic probes. 


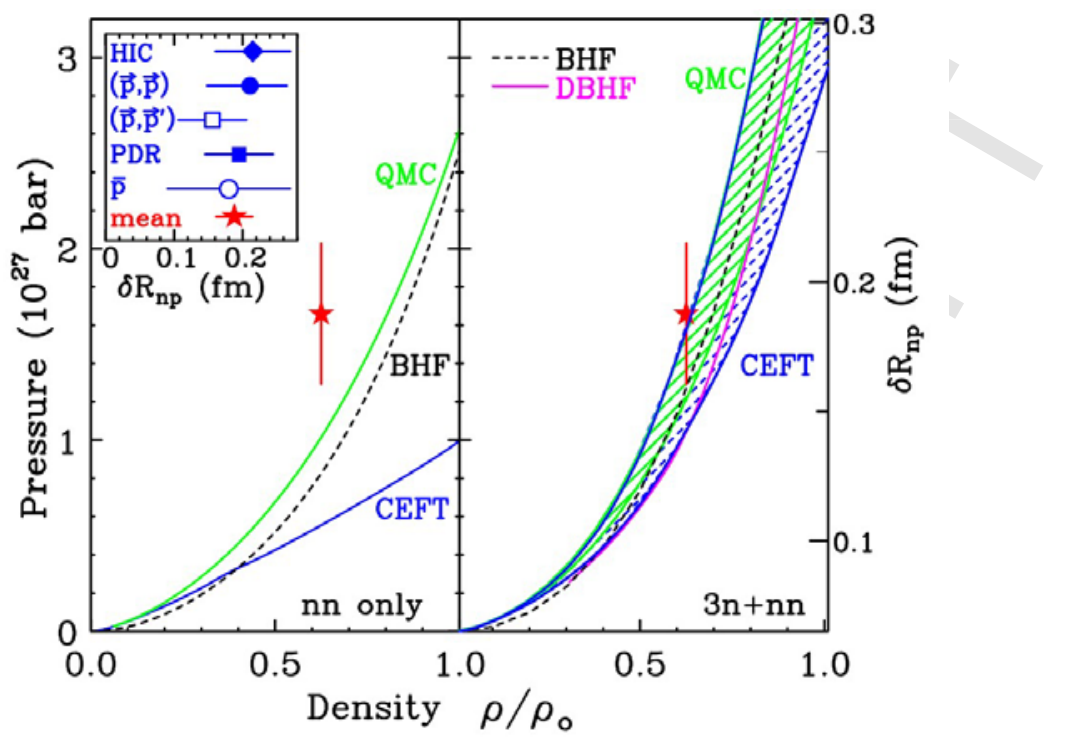

Figure 4: Density dependence of pressure in pure neutron matter as predicted in several models, (BruecknerHartree-Fock (BHF), Quantum Monte Carlo, and chiral effective field theory (CEFT), models without (left panel) and with (right panel) $3 n$ forces. The red star represents experimental data on the neutron skin thickness in ${ }^{208} \mathrm{~Pb}$ from measurements with hadron probe. (Figure taken from ref. [M. B. Tsang, et al., Phys. Rev. C 86, 015803 (2012)]

Results from the skin thickness measurements together with the analysis from the Giant Monopole Resonances as well as isobaric analog states constitute a diverse set of nuclear structure measurements that provide constraints to dependence of symmetry energy at subsaturation density. Heavy-Ion collisions which explore the dynamics and transport of isospin contents between projectile and target (known as isospin diffusion) provided one of the first experimental set of constraints on the density dependence of symmetry energy. Consistent constraints have been obtained from both the nuclear structure and reactions.

Above the saturation density, in addition to the density dependence of the symmetry energy, the momentum dependence of the nucleon symmetry potentials become important. Since the $n$ and $p$ symmetry potentials have opposite signs, emission of a particular nucleon type is enhanced or suppressed depending on the asymmetry. The issue of the momentum dependence of the symmetry potentials can be recast in terms of nucleon effective masses, where the difference in potentials results in an effective mass splitting of nucleon types that increase strongly with density. Heavy-ion collision experiments with Sn and $\mathrm{Ca}$ isotopes to measure the effective mass splitting using neutron-to-proton spectral ratios at higher densities have provided sufficiently accurate experimental results to distinguish between transport models with different effective mass splitting. These experimental results may also yield information on shortrange interactions between nucleons.

Insight into the effective mass difference at saturation density and below can be obtained within the context of the Dispersive Optical Model (DOM) analyses. The DOM cannot be extended to high density, but can be made more robust in the region of saturation by extending the asymmetry range of the experimental scattering data. This will create an important touchstone for heavy-ion reaction dynamics studies that can probe higher densities. 


\subsubsection{Near-Term and Long-Term Resources Needed}

\subsubsection{Accelerator Facilities}

Creation of dilute and dense nuclear matter requires studying collisions of nuclei over a large energy range and will involve many of the low-energy facilities in the USA. The sensitivity to the symmetry energy increases as the square of the asymmetry of the reaction system. Thus high-intensity radioactive ion beams are the key to the EOS efforts discussed here. The enhanced sensitivity offered by radioactive beams is essential for reliable extrapolations to the extreme neutron-rich neutrinosphere of supernovae. The radioactive-ion-beam capabilities of TAMU, NSCL and in the future, FRIB are essential for this effort. Smaller laboratories, like FSU, will also play pivotal roles in focused experiments such the fusion of exceedingly neutron-rich light nuclei. Facilities such as LANSCE, Ohio and TUNL, capable of producing neutrons, the lightest radioactive beam, are also essential for collecting data to buttress the DOM analysis. Similarly, proton elastic scattering data from nuclei off of stability will be helpful for DOM based extrapolations to the drip line. Alpha-clustering occurring at the surface of heavy nuclei where the nuclear density is low can be systematically studied by reactions like (p,p' $\alpha$ ) and surface dominated reactions like (d, $\left.{ }^{6} \mathrm{Li}\right)$ across long isotopic chains.

In addition to the PREXII (a second generation of ${ }^{208} \mathrm{~Pb}$ neutron skin thickness measurement at JLab) and CREX (skin thickness measurements of ${ }^{48} \mathrm{Ca}$ ) experiments, there are parallel global efforts to measure the skin thicknesses over a wide range of isotopes by measuring the polarizability at RCNP, Pygmy Dipole Resonances at GSI, HI $\gamma \mathrm{S}$, and RIKEN and photo production of $\pi^{0}$ at Mainz. These efforts complement JLab experiments and have the potential to provide bench-mark data for cross-comparisons and systematics of neutron radii over wide-range of isotopes. Due to the strong correlation between the symmetry energy and its slope at saturation energy, theoretical analyses must be conducted on all experiments to understand the ambiguities of any measurement and to determine realistic error bounds.

\subsubsection{Detectors and Other Experimental Necessities}

Since the intensities of the radioactive beams are not as high as those of stable beams, having high efficiency detectors is of paramount importance. Time Projection Chambers (TPC) satisfy this need and have the flexibility to be used across a broad energy, and thus density range. These will be employed in experiments both at RIKEN and NSCL. These instruments will not only be used in reaction dynamics experiments but also to extend the study of the monopole resonance and fission barriers with radioactive beams. Fission properties of very neutron-rich subactinides can be studied with a TPC. These properties are dictated by the asymmetry of the low-density surface and can provide knowledge required to understand fission recycling that could be important during r-process nucleosynthesis.

The specialized equipment required for many projects does not fit into the major equipment rubric. The study of the fusion of very neutron-rich nuclei, measuring neutron total cross sections on rare but stable isotopes and secondary-beam elastic scattering require healthy research funding for small and modest sized groups.

One issue that is generic is the need for significant quantities of rare but stable isotopes for targets. This need has compelled the EOS community to form a world-wide consortium for sharing the required isotopes. The amount of material for the experiments that probe high density or do n-scattering, is generally two orders of magnitude more than that used in low-energy charged-particle reactions. Extending the already existing international collaboration efforts are also needed to make optimal use of forefront radioactive ion beam facilities around the world, i.e. RIKEN, GSI and GANIL. 


\subsubsection{Reaction Modeling}

Close collaboration between theory and experiment is necessary to design, interpret, and extract nuclear matter properties from heavy ion collision experiments. For example, more than a decade ago it was predicted that pion yield ratios should be more sensitive to the symmetry energy at high density than $\mathrm{n} / \mathrm{p}$ yield ratios. Radioactive beams can provide reaction systems with wider range of isospin asymmetries. However, even for the accessible systems with current and planned radioactive beam facilities, the symmetry energy constitutes less than $10 \%$ of the total energy in the system. The strategy, successful in the past and to be used in the future, is to use pairs of reactions with similar proton numbers, to reduce the difference in Coulomb effects, but different neutron numbers to enhance the symmetry energy effects. Laboratory measurements of the charged pion spectra (as a function of beam energy) with a TPC for systems of greatly different asymmetries can shed light on the symmetry energy. Illustrated in Fig. 5 are the predictions of the differences between $\pi^{-} / \pi^{+}$yield ratios for ${ }^{132} \mathrm{Sn}$ (beam) $+{ }^{124} \mathrm{Sn}$ and ${ }^{108} \mathrm{Sn}$ (beam) $+{ }^{112} \mathrm{Sn}$ systems showing the sensitivity to the density dependence of symmetry energy. More importantly, this new proposed observable, that requires measurements of the charged pion energy spectra, appears to be more sensitive than the original proposed pion yield ratios to the symmetry energy. The calculations also predict the interplay of different effects. Symmetry energy, Coulomb energies, pion optical potentials, and pion production thresholds all come into play especially at low pion energy. Measurements at different beam energies will be needed to resolve some of these ambiguities.

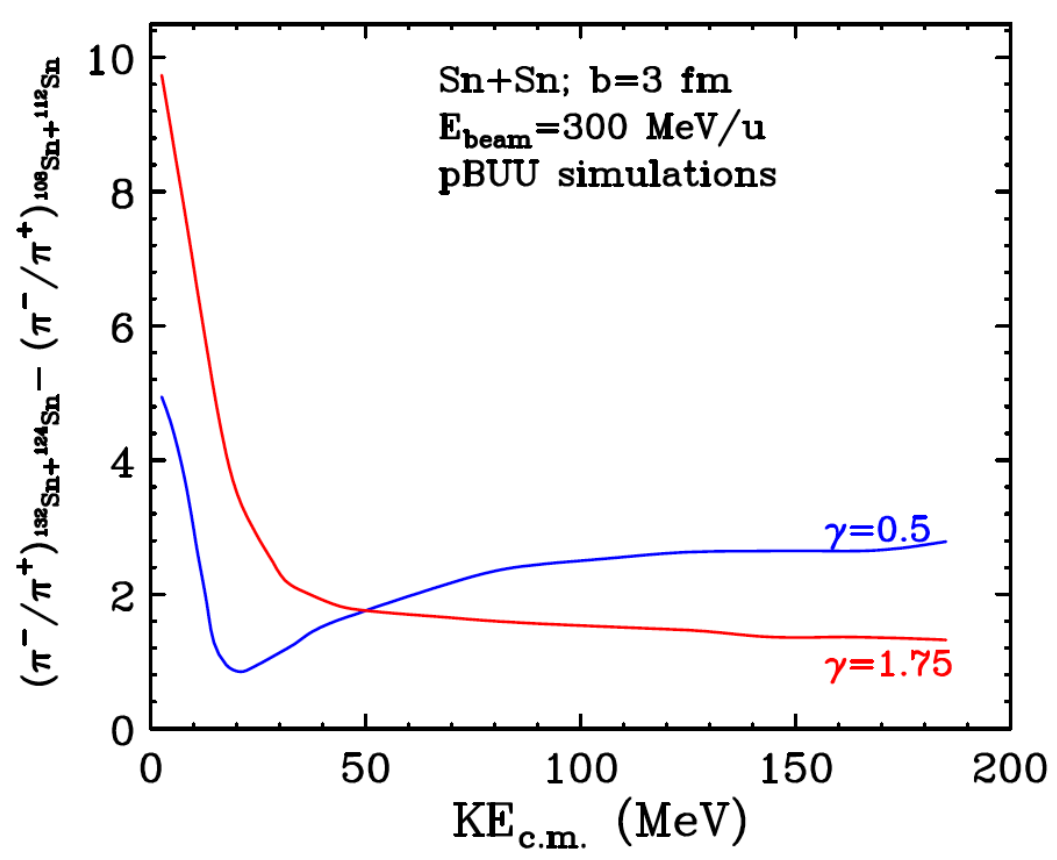

Figure 5: Differences between $\pi^{-} / \pi^{+}$spectra ratios for ${ }^{132} \mathrm{Sn}($ beam $)+{ }^{124} \mathrm{Sn}$ and ${ }^{108} \mathrm{Sn}(\mathrm{beam})+{ }^{112} \mathrm{Sn}$ systems at incident energy $300 \mathrm{MeV}$ per nucleon as predicted by Boltzmann Uehling Uhlenbeck transport calculations. The $\gamma$ values indicates the stiffness of the symmetry energy. Large $\gamma$ values indicates larger symmetry energy at high density or stronger density dependence.

Above the saturation density region, the momentum dependence of the symmetry potential also becomes significant. Recent transport calculations predict that the beam energy range between 100 to $200 \mathrm{MeV}$ per nucleon is optimal to study and isolate this momentum dependence. The interplay between experiments and phenomenological calculations is essential for progress. During the next long range plan period, extensive theoretical work quantifying systematic errors in transport models, and determining the effects of these uncertainties on possible symmetry energy observables, should be undertaken. 


\subsubsection{Scientific Impact on Other Fields and Interdisciplinary Connections}

The properties of nuclear matter and the equation of state are very important in astrophysics. We give some selected examples. First at low densities we discuss supernovae and nucleosynthesis and then at high densities we discuss neutron star structure and neutron star cooling. We end with a discussion of dense QCD.

Supernovae and neutrinosphere properties: Core collapse supernovae are large explosions of massive stars that synthesize chemical elements, accelerate cosmic rays, and form neutron stars and black holes. The explosion dynamics is sensitive to neutrino interactions and other properties of the medium near the neutrinosphere. Accurate compositions, equations of state, symmetry energies, and neutrino interactions are needed for warm low-density matter in order to constrain the important microphysics for large-scale supernova simulations.

The site(s) of r-process nucleosynthesis: Half of the heavy elements are believed to be made in the rprocess where heavy neutron-rich nuclei rapidly capture many neutrons (before there is time for beta decay). Predicted abundances may depend on the masses and beta decay half-lives of these nuclei. However abundances also depend, perhaps much more strongly, on bulk properties of neutron-rich matter and on conditions in astrophysical sites.

Possible r-process sites include the neutrino driven wind in core collapse supernovae and the ejected material during neutron star mergers. As discussed above, the ratio of neutrons to protons in the neutrino driven wind depends on the spectra of emitted neutrinos (that capture protons to make neutrons) and antineutrinos (that destroy neutrons to make more protons). These spectra in turn depend on the symmetry energy and other properties of dilute neutron-rich matter. Presently it is thought that the neutrino driven wind may not be neutron rich enough to synthesize heavy ( $3^{\text {rd }}$ peak) r-process elements such as gold or uranium. Alternatively, ejected material during neutron star mergers is thought to be very neutron rich because it comes from high-density neutron star material.

Neutron-rich material can be ejected during the neutron star mergers from gravitational tides, from dynamical ejection of high pressure colliding matter, and from the evaporation of an accretion disk. These mechanisms, and the total amount of material ejected, are sensitive to the EOS and other properties of neutron-rich matter. For example a stiff, high pressure EOS could lead to more tidal ejecta while a soft EOS may have more ejecta from colliding matter. An infrared glow from the radioactive decay of newly synthesized r-process material, during a neutron star merger, may have been recently detected as a "kilonova".

The contribution of neutron star mergers to the r-process inventory of the galaxy depends on the product of the rate of mergers and on the amount of material ejected per merger. The rate of neutron star mergers should soon be directly measured with the Advanced LIGO gravitational wave detectors. These are presently being upgraded and, when operational, are expected to detect a few or more neutron star mergers per year.

Neutron star structure: The symmetry energy at high densities is the single laboratory observable most directly relevant to determine the structure of neutron stars. The symmetry energy helps determine the pressure of dense neutron-rich matter and hence the radius of neutron stars. A high pressure holds the star against gravity and leads to a large radius. Furthermore, the symmetry energy determines the difference between the neutron and proton chemical potentials. This chemical potential difference is the driving force for including additional hadrons such as hyperons, meson condensates, or various quark phases into the neutron star core. A large symmetry energy could favor the presence of exotic hadrons in neutron stars. There are many, somewhat controversial and potentially model dependent, efforts to determine neutron star radii from $\mathrm{x}$-ray observations. 
Neutron star cooling: Neutron stars cool by neutrino emission from their high-density cores. For example they can cool via the interconversion (beta decay) of neutrons and protons (the URCA process). This reaction rate is slow when a second spectator nucleon is needed to conserve momentum (standard URCA), and fast when no spectator (direct URCA) is needed. For the fast process to occur the proton fraction must be greater than about 0.11 and for this to be the case the symmetry energy must be high. Alternatively, if direct URCA is ruled out, neutron stars could cool quickly by the beta decay of additional hadrons (besides neutron and protons) such as hyperons, meson condensates or quarks. There is one observation of a quiescent neutron star with a luminosity which suggests some sort of enhanced cooling. If the planned laboratory heavy-ion reaction studies can measure the symmetry energy and rule out direct URCA, the observation of enhanced cooling suggests the presence of additional hadrons, beyond neutrons and protons, in the core of the star.

Dense QCD: Neutron star observations are one of the few feasible ways to study cold dense matter. This is made more important because at present we have no reliable way to calculate properties of cold dense matter. Likewise heavy-ion collisions at intermediate energies provide the only laboratory way to study (warm) dense matter. What are the high-density phases of QCD? Do they involve hadrons or nearly free quarks? And if quarks, are they paired into a color superconductor? Recently, relativistic heavy-ion collisions have discovered a strongly interacting quark gluon plasma phase of hot dense matter. Indeed the interactions between quarks and gluons appear to be so strong that the partons transport almost no momentum and the shear viscosity is found to be very low. If the partons are strongly interacting in hot matter then they may also be strongly interacting in cold dense matter. Therefore the interior of a neutron star may be very far from a weakly interacting color superconductor phase. However, at this time the actual form of cold dense matter is largely unknown and we do not know what a neutron star is made of. Observations of neutron stars and heavy-ion collisions during the next decade should significantly increase our knowledge of cold dense matter and of the phases of dense QCD. 


\subsection{Facilities and Instrumentation}

\subsubsection{Infrastructure and Resources in Nuclear Structure and Nuclear Astrophysics}

Reaching the goals of the field of low-energy nuclear physics requires access to mixtures of rare isotopes, higher intensity ions closer to stability, and stable beams. The total available beam time must be sufficient to support the community to perform world-leading research while training the next-generation workforce. This research requires higher sensitivity and precision and this in turn, implies improved instrumentation.

Improved accelerator facilities and instrumentation always lead to new discoveries and construction of the Facility for Rare Isotope Beams (FRIB) will add tremendous discovery potential in nuclear structure, reactions, and astrophysics. Its construction is the top priority of the field. This new facility will build on the rich world-leading physics programs that exist at the current facilities, which are essential for preparing the community for FRIB. We expect these facilities to continue to play an important role in the FRIB era. A number of experimental developments are on-going to improve the existing capabilities that will translate directly into improved experimental systems for FRIB. Thus, it is absolutely essential to adequately fund continued operation and design and construction of instrumentation at existing facilities.

The current stable and radioactive beam facilities with their state-of-the-art instruments have been very productive in the last five or so years. However, due to budget constraints, the community has lost a number of facilities and the remaining facilities are heavily oversubscribed. Appropriate funds and small upgrades to existing national and university laboratories are required to increase the effectiveness of these facilities.

\section{National Laboratory and University Accelerator Facilities}

The accelerator facilities located at national laboratories and universities are the backbone of nuclear structure and nuclear astrophysics research in the U.S. These facilities focus on many different aspects of research in nuclear structure and nuclear astrophysics and provide a broad and coherent program. The optimal utilization of these facilities is crucial for a healthy community of researchers and students that will be ready when FRIB is operational.

\subsubsection{ATLAS at ANL}

ATLAS is the DOE National User Facility for nuclear physics research at Coulomb barrier energies and above. It provides a wide range of beams for nuclear reaction and structure research to a large community of users from the US and abroad. The full range of all stable ions can be produced in ECR ion sources, accelerated up to $10-20 \mathrm{MeV} /$ nucleon in the world's first superconducting linear accelerator for heavy ions and delivered to one of several target stations. ATLAS also provides light radioactive beams close to stability through in-flight production and very neutron-rich reaccelerated beams from the CARIBU upgrade.

ATLAS delivers with high reliability beams for research for about 5500 hours per year. Of these, roughly 2000 hours per year are used for in-flight produced and reaccelerated CARIBU radioactive beams. An additional 2500 hours per year of low-energy neutron-rich beams is provided by CARIBU in stand-alone 
mode. Many of the radioactive beam species are from refractory species not easily available elsewhere in this energy regime.

The suite of research instruments available at ATLAS enables a wide variety of nuclear science programs from stopped beam measurements with ion and atom traps, measurements of cross sections of reactions at energies relevant to nuclear astrophysics, and nuclear structure and reaction mechanism studies at energies at or well above the Coulomb barrier. The present instruments include: the Fragment Mass Analyzer (FMA) and associated detectors; Digital Gammasphere and the Xarray for $\gamma$-ray detection; the HELIOS helical orbit spectrometer for single-particle structure studies; two split-pole magnetic spectrographs and associated detectors used for astrophysics, AMS measurements, fusion reaction studies; the CPT Penning trap mass spectrometer and the beta-decay Paul trap with their associated rare isotope beam manipulation systems; and other general-purpose detectors and beam lines. This array of state-of-the-art instruments is ideally suited for precision measurements.

The ATLAS superconducting linac has been recently upgraded for very high heavy-ion beam intensity and increased efficiency. The maximum beam intensity is essentially limited by ion source performance and can exceed $10 \mathrm{p} \mu \mathrm{A}$ at Coulomb barrier energies. On-going upgrades include an EBIS charge-breeder to increase the intensity and purity of CARIBU reaccelerated beams, the dedicated recoil separator AIRIS to increase the intensity of the in-flight radioactive beams, the gas-filled spectrometer AGFA to improve the transport efficiency of rare reaction recoils, and the $\mathrm{N}=126$ factory to produce and separate isotopes in the unexplored neutron-rich region with $\mathrm{N}>126$ that is responsible for the last peak in abundance of the $\mathrm{r}$ process.

Although the ATLAS/CARIBU facility provides roughly 8000 hours for research per year, the facility is highly oversubscribed with a ratio of requested-to-accepted beam time to the ATLAS PAC of roughly 3. A multi-user upgrade is currently being contemplated for ATLAS to provide an additional 2000 to 3000 hours per year to its users. It takes advantage of the additional flexibility provided by the recent and ongoing upgrades to ATLAS to accelerate simultaneously a radioactive beam and a stable beam, both with essentially full efficiency.

\subsubsection{National Superconducting Cyclotron Laboratory}

The NSF funds operation of the National Superconducting Cyclotron Laboratory, NSCL, which is one of the nation's flagship user facilities with over 1300 registered users. The primary research mission is to understand the nature of the nuclear force, structure of atomic nuclei, and the origin and evolution of chemical elements in the universe using beams of rare isotopes. Rare isotopes are produced in-flight from beams of stable ions ranging from helium up to uranium, energies up to $160 \mathrm{MeV} / \mathrm{u}$, and beam power of up to $1 \mathrm{~kW}$. NSCL's Coupled Cyclotron Facility (CCF) has produced over 1000 rare isotope beams for research and typically has operated at between 4000 and 5000 hours per year. Research capabilities include stopped-beam facilities for trapping and laser spectroscopy, reaccelerated beam experiments at astrophysical energies, and fast beam equipment such as the S800 magnetic spectrograph that can be coupled with GRETINA. Modest applied programs are in biology, materials studies, and radiation effects in semiconductor devices.

A significant upgrade has been completed with the addition of a 3-6 MeV/u post accelerator, ReA3, which will reaccelerate the rare isotope beams. This facility will supply beams of rare isotopes with short half-lives or chemical properties that may not be available at ISOL facilities. ReA3 enables research at energies close to the Coulomb barrier, e.g., Coulomb excitation, capture and transfer reactions. It also will enable a new set of experiments to determine important reaction rates for explosive stellar nucleosynthesis. Initial experiments have been completed. 
Major issues at the NSCL include the high demand for beam time from the user community. Operation at the 4000 to 5000 hour/year level is critical for advancing the science of nuclei and nuclear astrophysics while FRIB is being constructed. Another compelling opportunity is the upgrade of the reaccelerator to 6 to $12 \mathrm{MeV} / \mathrm{u}$. This would allow the novel reaccelerated beams of the facility to be used for direct reaction studies to probe single-particle structure, fusion-evaporation reactions to explore paths to superheavy elements, deep inelastic reactions with neutron-rich beams to study heavy neutron-rich nuclei, surrogate reactions for astrophysics and security, and fission barrier studies over a wide range of the chart of nuclides.

\subsubsection{Low-Energy University Based Facilities (ARUNA)}

The university-based ARUNA laboratories contribute a diverse portfolio of research instruments and programs to the nuclear science community. Some of the facilities provide unique capabilities, not available at the national user facilities, such as beams of energetic neutrons, $\gamma$-rays, and high-intensity low-energy ion beams. Other facilities perform experiments, which, because of their duration and character, are not often done at the national user facilities.

The John D. Fox Laboratory at Florida State University operates a 9 MV Tandem accelerator injecting into a superconducting linear accelerator, adding another $9 \mathrm{MV}$ potential for heavy ion beams. The laboratory pursues research programs in high-resolution $\gamma$-spectroscopy, using an array of 3 Clover and 10 conventional anti-Compton Germanium detectors. The RESOLUT in-flight rare ion beam facility produces beams between mass 6 and 30 and is used in experiments of nuclear structure and nuclear astrophysics. The laboratory has begun installing a split-pole spectrograph to enable new research programs in nuclear astrophysics and nuclear structure of open systems. The laboratory is also in the process of upgrading the LINAC.

The Hope College, Ion Beam Analysis Laboratory is pursuing a program in materials analysis with a 1.7 MV Tandem Pelletron accelerator. Research includes environmental applications, as well as art and archeology.

The John E. Edwards Laboratory at Ohio University operates a 4.5 MV T-type Pelletron-charged Tandem accelerator which delivers high-intensity beams of $\mathrm{p}, \mathrm{d},{ }^{3} \mathrm{He}$ and ${ }^{4} \mathrm{He}$ as well as heavy ion beams. The laboratory supports programs in light-ion reactions, statistical physics and nuclear astrophysics. The laboratory is especially well equipped for measurements with neutrons, using a $30 \mathrm{~m}$ long underground tunnel for high resolution time-of-flight experiments.

The Texas A\&M Cyclotron Laboratory operates a K-500 superconducting Cyclotron and a K-150 Cyclotron, producing heavy-ion beams from two ECR-sources or a negative ion source. The laboratory supports experiments with radioactive beams at the MARS separator, with impact on nuclear astrophysics, the structure of exotic nuclei and high-precision $\beta$-decay studies. Other programs include the study of the nuclear equation of state in heavy-ion reactions with the NIMROD neutron and chargedparticle detector system, the study of the breathing mode of nuclei as well as the study of the synthesis of super-heavy nuclei. New experimental initiatives include the TAMU-trap for the precision study of $\beta$ delayed proton decays and the Aggie gas-filled spectrometer for the chemistry of super-heavy elements. The laboratory is progressing towards the T-REX upgrade, coupling both Cyclotrons to produce, stop and re-accelerate exotic nuclei.

The Triangle Universities Nuclear Laboratory (TUNL) operates three facilities as a consortium of Duke University, University of North Carolina, and North Carolina State University. The TUNL-HI $\gamma$ S facility is the world's most intense Compton $\gamma$-ray source in terms of $\gamma / \mathrm{s} / \mathrm{keV}$, delivering a beam of more than $10^{6} \mathrm{\gamma} / \mathrm{s} / \mathrm{keV}$ to target in the energy range from 1 to $100 \mathrm{MeV}$. The 
electron accelerator drivers for $\mathrm{HI} \gamma \mathrm{S}$ consist of a linac pre-injector, a booster and a race-track shaped storage ring, producing electron beams in an energy range from $180 \mathrm{MeV}$ to $1.2 \mathrm{GeV}$. The $\gamma$-ray beam is produced by Compton back-scattering of the photons inside the optical cavity of the Free Electron Laser (FEL) from the circulating electron bunches in the storage ring. The $\gamma$-ray beams can be produced with circular or linear polarization of more than 95\%. Research programs include measurements of $\gamma$-induced astrophysical reaction rates and collective nuclear structure, using high-resolution $\gamma$-resonance fluorescence and photofission. The lab is pursuing an upgrade to increase the $\gamma$-ray energy to $120 \mathrm{MeV}$. The HI $\gamma \mathrm{S} 2$ project is designed to provide a 100 -fold increase in $\gamma$-ray intensity using an optical cavity, pumped with an external laser.

The TUNL-Laboratory for Experimental Nuclear Astrophysics (LENA) is a facility dedicated to nuclear astrophysics experiments using two electrostatic light-ion, high-intensity accelerators. An Electron Cyclotron Resonance (ECR) source on a 200-kV isolated potential platform, is capable of delivering proton and deuteron beams with currents of up to $2 \mathrm{~mA}$. The 1-MV JN Van de Graaff accelerator can deliver beam currents up to $200 \mu \mathrm{A}$. Research is aimed at the direct measurement of astrophysical reaction rates with relevance to stellar evolution, Novae and x-ray bursts. Upgrade plans include replacement of the $\mathrm{JN}$ with a more modern accelerator.

The TUNL-Tandem laboratory operates a 10 MV Tandem Van de Graaff, producing beams of p, d, ${ }^{3} \mathrm{He}$ and ${ }^{4} \mathrm{He}$, as well as polarized protons and deuterons. A special feature of the facility is the nearly mono-energetic, unpolarized or polarized fast neutron beams. Research programs in few-body reactions are pursued in general-purpose charged-particle scattering chambers and neutron time-of-flight (TOF) systems. A new initiative to measure resonance spectra for nuclear astrophysics is will employ an Enge split-pole magnetic spectrometer.

Union College, Ion Beam Analysis Laboratory operates a 1.1 MV Tandem accelerator for ion beam analysis, applied to research in environmental sciences.

The University of Kentucky Accelerator Laboratory (UKAL) operates an upgraded 7 MV singleended Van de Graaff accelerator producing pulsed beams of p, d, ${ }^{3} \mathrm{He}$ and ${ }^{4} \mathrm{He}$ for the in-flight production of mono-energetic neutron beams. A program is pursued in inelastic neutron-scattering that can be combined with high-resolution $\gamma$-spectroscopy to study low-lying collective excitations, such as multiphonon states and excitations with proton-neutron asymmetry.

The University of Massachusetts-Lowell Radiation Laboratory operates multiple facilities: a 1 MW research reactor, a hot-cell with remote manipulators, a $100-\mathrm{kCi}{ }^{60} \mathrm{Co}$ source, and a $5.5 \mathrm{MV}$ single-ended Van de Graaff accelerator. The laboratory is supporting the development of neutron and segmentedGermanium detectors and includes a materials characterization program using PIXE with a proton microbeam.

At the University of Notre Dame, the Institute for Structure and Nuclear Astrophysics (ISNAP) currently operates three accelerators. An 11 MV FN Tandem accelerator drives the TwinSol in-flight radioactive beam facility for studies of light exotic nuclei and nuclear astrophysics and is also used as an accelerator-mass spectrometer. The recently installed (2012) St.Ana single-ended 5 MV Pelletron accelerator produces high-intensity beams from an ECR ion source and measures nuclear reaction rates with the new St. George recoil separator. A 1 MV JN Van de Graaff accelerator will be part of the 'Caspar' laboratory at the Sanford Underground Research Facility and is described below. A new project at ISNAP is based on a $24 \mathrm{MeV}$ medical cyclotron, to measure beta-delayed neutron emission in neutronrich nuclei. 
The Center for Experimental Nuclear Physics and Astrophysics (CENPA) at the University of Washington uses its 10 MV FN Tandem accelerator to produce laser-trapped ${ }^{6} \mathrm{He}$ isotope with worldrecord intensities for precision angular correlation measurements of its $\beta$-decay.

\subsubsection{Thomas Jefferson National Accelerator Facility}

Thomas Jefferson National Accelerator Facility (JLab) is operated by the U.S. Department of Energy as one of the nation's flagship user facilities and serves a user community of approximately 1300 scientists. The lab's primary mission is to conduct basic research on the structure of atomic nuclei, the nature of how nuclei and nucleons are bound by QCD, the internal structure of the nucleon, and make tests of the Standard Model. These programs are enabled using the lab's unique electron accelerator, named the Continuous Electron Beam Accelerator Facility (CEBAF). CEBAF is able to deliver high-current, continuous beams of polarized or un-polarized electron beams to up to four experimental halls simultaneously. In 2014 the upgrade of CEBAF to $12 \mathrm{GeV}$ was completed and a new experimental Hall, Hall D, was opened. Jefferson Lab also conducts a variety of research using its Free-Electron Laser, which is based on the same electron-accelerating technology used in CEBAF. The laboratory contributes to accelerator projects nationwide including FRIB and LCLS II at SLAC.

As part of its research profile, JLab conducts experiments directed at study of nuclear structure. The primary aim is understanding the short-range part of the nuclear interaction, but also measures precision data such as charge and neutron radii of nuclei, in-nucleus form factors, and nucleon momentum distributions. The program uses spectrometers in Halls A and C, and the CLAS12 device in Hall B. The GLUEX system located in the new Hall D is used to probe the nature of QCD at short range and search for exotic mesons. High-profile experiments PREX and CREX use the sensitivity of the weak interaction to neutrons to determine neutron radii in ${ }^{208} \mathrm{~Pb}$ and ${ }^{48} \mathrm{Ca}$ by parity violating electron scattering. Spectral functions of nucleons in the nucleus are studied to test $a b$-initio nuclear structure calculations, provide input into neutrino cross sections for oscillation experiments, and study the long-range correlations of nucleons in nuclei. The degree and nature of short-range correlations of nucleons in nuclei are studied and the degree to which nucleons in the nucleus are modified is addressed. JLab also has a program to produce and study the spectroscopy of Hypernuclei.

\subsubsection{LANSCE}

The Los Alamos Neutron Science Center is a facility with multiple neutron flight-paths driven by 800$\mathrm{MeV}$ proton-induced spallation on tungsten, providing intense time-of-flight neutrons from subthermal to over $400 \mathrm{MeV}$. Both moderated and unmoderated spallation targets are operated with independent nuclear science flight-paths on each, allowing parallel experiments. Existing instrumentation is focused on the measurement of $(n, \gamma),(n, n)$, (n,fission), $(n, p)$, and $(n, \alpha)$ reactions with the DANCE $B^{2} F_{2} \gamma$-ray calorimeter, the GEANIE HPGe array, a fission TPC, the Chi-Nu neutron detector array, the SPIDER fission product spectrometer, and the LENZ silicon array. This suite of instruments enables measurements for basic and applied nuclear physics improving the understanding of nuclear structure and reaction models.

\subsubsection{Future Facilities and Instrumentation}

There is consensus within the low-energy nuclear science community that a major U.S. investment in FRIB dedicated to the study of nuclear science with radioactive ion beams is vital to maintain the health 
of our field. Leadership-class experimental instruments are also essential to our future and to the effective and efficient utilization of FRIB, ATLAS, and other facilities in the field. This requires that development of instruments (and beam delivery capabilities) at existing facilities be supported vigorously; the suite of instruments developed or improved over the next decade will form the basis of the FRIB program at startup.

\subsubsection{Facility for Rare Isotope Beams}

The Facility for Rare Isotope Beams, FRIB, will be the world's most powerful radioactive beam facility, making nearly $80 \%$ of the isotopes predicted to exist for elements up to uranium. FRIB capabilities will provide unprecedented opportunities to study the origin and stability of nuclear matter. It will be an international-class user facility, which will provide U.S. researchers a location to base major programs and play leading roles in equipment operation and development, and define the future directions of the field.

FRIB will be the world's leading facility for addressing key nuclear science challenges articulated in this document, the 2007 NSAC LRP, and the NRC2012 report. With its ability to deliver the full elemental variety of reaccelerated beams, most of the reaction rates of astrophysical importance involving radioactive ions can be measured. It will be possible to carry out studies of a wide range of nuclei at the very limits of nuclear stability where specific aspects of the nuclear many-body problem can be explored. The unique features of FRIB will allow the delineation of the proton or neutron limits of existence to higher masses than other facilities. It will double the number of accessible neutron-rich nuclei leading to new information about matter with unusual features such as halos, skins, and their new collective modes. FRIB will provide the U.S. community with rare isotopes that are crucial for the exploration of fundamental symmetries. The accelerator's high power will yield the greatest number of different isotopes produced anywhere in the world, thereby allowing the possible r-process sites and the respective paths to be determined.

What will make FRIB unique in the international context is the use of a $200 \mathrm{MeV} / \mathrm{u}, 400 \mathrm{~kW}$ heavy-ion driver linac, whose power level, and consequent intensity and reach, is unmatched at other existing facilities, or those now under construction, around the world. FRIB will provide intense beams of rare isotopes through in-flight fragmentation and fission of fast heavy-ion beams combined with gas stopping and reacceleration. A key feature is the capability to reaccelerate ion beams after stopping in gas. Beams not readily available at ISOL facilities can be produced and nearly any isotope can be used with limited development time.

The full complement of fast, stopped, and reaccelerated beams will be available for experiments with a broad suite of equipment. Most of the equipment complements that prepared at ATLAS, NSCL and other low-energy facilities world-wide and is described in this report.

FRIB is scheduled for a CD4 date in 2022 with a scheduled completion in 2020. In the longer term after FRIB is completed there are a number of key upgrade possibilities. Space has been left in the LINAC tunnel to accommodate additional cryomodules and raise the production energy to $400 \mathrm{MeV} / \mathrm{u}$. This critical upgrade would increase the secondary beam yields by an order of magnitude and provide a wider reach for r-process studies. Other upgrade options supported by the science are an ISOL production target, a second injector for simultaneous beam operation, and a doubling of the experimental floor space for future research equipment.

\subsubsection{Equipment Development for Low-Energy Nuclear Physics}

The development of new reaccelerated beam capabilities from CARIBU at ATLAS, ReA3 at NSCL and soon T-Rex at Texas A\&M, together with increased in-flight production at ATLAS and Florida State 
University, in addition to increased stable beam capabilities, provide unique opportunities for forefront research with improved instrumentation. Moreover, although any new facility should be equipped with state of the art new equipment, the situation for FRIB differs in some ways from other major nuclear physics facilities that were brought online over the last years. At both RHIC and CEBAF none of the existing detectors were adequate and the design and construction of new detectors had to move forward simultaneously with the construction of the accelerator in order to produce scientific results when the first beam became available.

FRIB will produce rare isotope beams at similar energies as those used at current facilities. For example, most of the existing experimental equipment at the NSCL CCF is designed for rare isotopes produced by $200 \mathrm{MeV} /$ nucleon primary beams. The reaccelerated beam program at FRIB will have essentially the same overall detector requirements as current systems at Texas A\&M, ANL or FSU. The techniques and the intensities for the rare isotopes are the same. The main and most important difference is that isotopes much further from the line of stability will become accessible. Thus the equipment currently in use or being developed at operating facilities is often well suited to be used at FRIB. For example, SeGA, MoNA-LISA, HiRA, LEBIT, and BECOLA at NSCL and the CPT, APT, laser atom traps, split-pole spectrographs, HELIOS and the FMA at ANL are used routinely for rare isotope experiments. Hence FRIB will benefit from past and current equipment investments. In addition, a limited set of new instruments will be necessary to realize the science of the rare isotope beams. These developments, listed below, are absolutely essential in order to achieve the scientific goals and ensure that state-of-the-art equipment will be available close to the time FRIB begins operation.

Additional capabilities for important programs that cannot be addressed at FRIB, such as the study of low-background very low-energy astrophysics reactions or the production of the heaviest neutron-rich isotopes, are essential and complementary to the FRIB effort and are also presented below. Finally, the community does have access to other facilities whose main purpose is not low-energy nuclear physics but whose capabilities allow complementary studies to be performed effectively. Those are also included below.

\section{GRETINA and GRETA}

As stated in the 2002 NSAC Long Range Plan, "The detection of gamma-ray emissions from excited nuclei plays a vital and ubiquitous role in nuclear science. The physics justification for a $4 \pi$ tracking array is extremely compelling, spanning a wide range of fundamental questions in nuclear structure, nuclear astrophysics, and weak interactions." First discussed in the 1996 NSAC Long Range Plan (LRP) and then recommended as a major new initiative in both the 2002 and 2007 LRPs, the $4 \pi$ Gamma-Ray Energy Tracking Array (GRETA) will be a powerful new instrument needed to accomplish a broad range of experiments that will play an essential role in addressing the intellectual challenges of our field of lowenergy nuclear science. This device, made of highly segmented germanium detectors, is capable of reconstructing the individual interaction positions of incident $\gamma$ rays. This is a major advance in the development of $\gamma$-ray detector systems and provides a large increase in sensitivity and resolving power over existing arrays.

The FRIB Science Advisory Committee (SAC) has consistently identified and endorsed GRETA as a top priority for instrumentation crucial to the success of FRIB. This is best exemplified in that the majority of the 17 "benchmark experimental programs" (introduced in 2007 by the NSAC Rare Isotope Beam Task Force, to help establish and evaluate capabilities for a future rare-isotope beam facility) rely on highresolution, high-efficiency in-flight $\gamma$-ray detection. Thus, GRETA will be central to fully utilize and maximize the physics opportunities at FRIB, using both fast-fragmentation and reaccelerated beams. It will also add significant new capabilities to existing facilities, such ATLAS. An updated White Paper (www.lecmeeting.org/whitepapers.html) was submitted to this Town Meeting, with examples chosen to 
highlight GRETA's broad impact in many of the benchmark experimental programs mentioned above. In addition, $\gamma$-ray tracking technology may find important applications for applied science, medicine, and homeland security.

The technology and the scientific impact of a $\gamma$-ray tracking array has already been demonstrated by GRETINA, a $1 \pi$ segmented Ge detector array employing the same signal decomposition tracking technology as needed for GRETA. In its first science campaign (July 2012 - July 2013), GRETINA at NSCL was used to address a broad range of physics including studies of shell evolution and weak nucleon binding, determining excitation energies and spectroscopic factors of astrophysical relevance, and benchmarking the treatment of weak interactions in modern shell-model Hamiltonians. GRETINA is currently operating at ATLAS/ANL and first experiments using reaccelerated radioactive ions from CARIBU have been performed. Preliminary cost and schedule estimates have been developed based on current (GRETINA) technology. With a goal of CD0 by the end of FY15, completion of the full array is anticipated in 2024.

\section{AGFA - The Argonne Gas-filled Fragment Analyzer}

The Argonne Gas-filled Fragment Analyzer (AGFA) is a state-of-the-art gas-filled separator being developed at ATLAS to be used for a wide range of studies, e.g. 1) in conjunction with Gammasphere for in-beam and calorimetric studies of trans-fermium nuclei, 2) in the stand-alone mode for studies of superheavy nuclei, isomers in heavy elements, and fast proton and alpha emitters along the proton drip-line, 3) together with a gas catcher to prepare beams of exotic radioactive ions for mass measurements and laser spectroscopy of trapped atoms, and 4) to study, via deep-inelastic reactions, heavy neutron-rich nuclei that are important in the r-process.

The design takes a novel approach by combining in a single magnetic element, $D_{m}$, the bending capabilities of a dipole with the horizontal focusing and higher order magnetic field components to correct for aberrations to achieve a narrow distribution of the reaction products on the focal plane. Vertical focusing is achieved by a single quadrupole located upstream of the main magnet. This $\mathrm{Q}_{\mathrm{v}} \mathrm{D}_{\mathrm{m}}$ design achieves larger solid angle acceptance than most current separators, yet it has a smaller image size and path length, which leads to higher efficiency for (a) collection of evaporation residues and (b) detection of their decay gamma-rays.

The project received DOE approval, will be constructed over 2.5 years, with commissioning taking place in the 1st quarter in 2017. The separator is designed such that Gammasphere can be placed at the target location with target to quadrupole distance of $\sim 80 \mathrm{~cm}$ resulting in a solid angle of $22.5 \mathrm{msr}$. Without the need for prompt gamma-detection, the target chamber can be moved to $40 \mathrm{~cm}$ upstream of the quadrupole giving a solid angle of $>40 \mathrm{msr}$. The implantation station at the focal plane will be shared with the FMA and includes a large double-sided Si strip detector that can be surrounded by 5 HPGe clover detectors such as those comprising the X-array. Signals from these detectors will be recorded using the digital readout system developed for the FMA.

\section{AIRIS - The Argonne In-flight Radioactive Ion Separator}

At present, ATLAS produces light radioactive beams at energies approaching $20 \mathrm{MeV} /$ nucleon and intensities up to a few times $10^{6}$ particles-per-second via the in-flight production method. Neutrondeficient beams up to $\mathrm{Ca}$ and neutron-rich beams up to mass twenty have been used for the nuclear astrophysics and nuclear structure programs through reaction cross section and transfer measurements using beams at the proper energies. Expansion of the availability, intensity, purity, and reach of the radioactive beams produced in-flight is underway through the construction of a dedicated mass separator, the Argonne In-flight Radioactive Ion Separator (AIRIS). AIRIS consists of three major components: a 
magnetic achromat for primary beam rejection through rigidity selection, a bunching/rebunching resonator to optimize secondary beam characteristics, and a Radio-Frequency (RF) Sweeper providing velocity filtering and further purification of the secondary beam. AIRIS will rely on rotating solid or flowing liquid targets to handle the high beam power which can be provided by the upgraded ATLAS facility.

AIRIS will have a factor of 100 increase in sensitivity relative to those radioactive beams currently available. The improvement factor translates into higher beam rates for isotopes which have been previously produced at ATLAS, as well as expansion of reach by one or more neutrons from stability and to far heavier masses then presently accessible. Secondary beams will be available to experimental stations currently inaccessible e.g., Gammasphere and the FMA. In addition to the nuclear astrophysics and single-particle structure programs, AIRIS will provide intense low-energy radioactive beams for fusion evaporation reactions to study high-spin structure of neutron-rich nuclei around ${ }^{60} \mathrm{Fe}$ as well as single-particle structures around ${ }^{100} \mathrm{Sn}$, and elastic and inelastic reactions to probe nuclear cluster states in light systems.

\section{ATTPC - The Active Target Time Projection Chamber}

The Active-Target Time-Projection-Chamber is a versatile device for studying an array of nuclear reactions and decays that are important for the study of nuclear structure, reactions, and the production of the elements in astrophysical processes. Active-target detectors are well suited for use with weak rareisotope beams due to the increases in luminosity and efficiency they afford. The AT-TPC has the ability to precisely image charged particle tracks. Examples of experiments that have been done are measurement of cross sections for resonant alpha scattering, ${ }^{6} \mathrm{He}(\alpha, \alpha),{ }^{10} \mathrm{Be}(\alpha, \alpha)$, and fusion studies with radioactive beams, ${ }^{10} \mathrm{Be}+{ }^{40} \mathrm{Ar}$, proton scattering in inverse kinematics, ${ }^{124} \mathrm{Sn}(\mathrm{p}, \mathrm{p})$, and decay studies involving multiple charged particles in the exit channel, ${ }^{12} \mathrm{C}\left(0^{+}{ }_{2}\right) \rightarrow 3 \alpha$. Measurements involving direct reactions are also envisioned for the near future. The advantages of the active-target method will allow us to further push the boundaries of what is possible for experiments with radioactive beams. Continual development of active-target technology will be needed to fully take advantage of the rare-isotope beams that will be available at both ATLAS and FRIB.

\section{CASPAR - The Compact Accelerator System for Performing Astrophysical Research}

The Compact Accelerator System for Performing Astrophysical Research (CASPAR) facility is an accelerator project for low-energy nuclear astrophysics experiments, located at $4850 \mathrm{ft}$ level of the Sanford Underground Research Facility. CASPAR will serve as a demonstrator project for the Dual Ion Accelerators for Nuclear Astrophysics (DIANA) facility. It is based on refurbishing a $1 \mathrm{MV}$ accelerator from University of Notre Dame and a re-circulating, windowless gas target designed and constructed at Colorado School of Mines. CASPAR will be operated by the South Dakota School of Mines and Technology to enable a research program to determine the neutron production rates in stars via the study of $(\alpha, n)$ reactions at stellar energy conditions. These reactions as sources of neutrons for the s-process have been a matter of intense debate. The main s-process is associated with the inter-shell helium burning in AGB stars and the subsequent helium flash. The main sources in this scenario are $13 \mathrm{C}(\alpha, n)$ in the intershell phase, and $22 \mathrm{Ne}(\alpha, n)$ in the helium flash. Initially, CASPAR will focus on the measurements of these two main neutron sources, but the technique can be used to the study of secondary neutron sources such as $25 \mathrm{Mg}(\alpha, \mathrm{n})$ that are anticipated for stellar carbon burning conditions.

\section{CATS - The Center for Accelerator Target Science}

The Physics Division at ANL operates a target development laboratory that produces targets and foils of various thicknesses and substrates for experiments performed at ATLAS and other accelerator facilities. The targets are prepared from materials and stable isotopes that are supplied in elemental form and stable 
compounds. Our community continues to express its concerns about sufficient availability of target fabrication capabilities in the U.S. The ANL Physics Division has proposed the establishment of the Center for Accelerator Target Science (CATS) to meet these needs and submitted the CATS proposal to the U. S. Department of Energy in October 2013. The proposal is strongly supported by the low-energy users community.

\section{Decay Spectroscopy Station at FRIB}

Decay spectroscopy is one of the primary methods to study exotic nuclei at FRIB and must handle event rates of many thousands per second for precision measurements to less than 1 per minute for exploratory physics. Often decay data is the first information known about an isotope and ranges from energy levels, half-lives, spins, parities, and decay transition strengths. Recent neutron-gamma correlation experiments indicate multiple-neutron emission in the $\beta$-decay of ${ }^{86} \mathrm{Ga}$ populates the $2^{+}$state in ${ }^{84} \mathrm{Ge}$. Thus, photon, neutron, and charge particle detector arrays must be combined together and optimized to provide the necessary selectivity to the complicated decay patterns expected from the new exotic isotopes produced at FRIB. Detector arrays made of "new scintillation materials" such as lanthanum bromide ( $\gamma$-rays) and CLYC $\left(\mathrm{Cs}_{2} \mathrm{LiYCl}_{6}: \mathrm{Ce}\right)$ (neutrons and $\gamma$-ray detection) be compatible with other arrays such as $\mathrm{Si}$ and segmented-Ge implantation detectors, Ge, $\mathrm{NaI}(\mathrm{Tl}),{ }^{3} \mathrm{He}$, and plastic detector arrays such that many combinations of different detectors can be used together. Total cost is expected to be $\$ 16.4 \mathrm{M}$ with components and full coverage built up over time. These detectors should be easily movable and used at the fragmentation stations as well as the low-energy, multi-user tape station. Due to the flexibility, many of the detector arrays could also be used in other experimental programs.

\section{HAGRID}

HAGRID is an array of lanthanum bromide scintillators to be combined with particle detectors such as VANDLE, 3Hen, or silicon detectors to provide excellent particle- $\gamma$ coincidence data for transfer reactions, $\beta$-decay, and charge-particle decay. Lanthanum bromide is a low cost alternative to high demand, high-resolution Ge detectors and provides adequate energy resolution suitable for low gamma multiplicity data typical from decay and transfer experiments. With its $\sim 300 \mathrm{ps}$ timing resolution it is a good match for experiments requiring time-of-flight. With lanthanum bromide's size and stack-ability, high efficiency particle- $\gamma$ coincidence detection is compact and cost effective. Total cost is scalable depending on coverage and efficiency and is estimated to be $\$ 300 \mathrm{k}$.

\section{HI $\gamma$ S Upgrade}

In the coming decade, upgrades of $\mathrm{HI} \gamma \mathrm{S}$ are planned to increase its energy reach to about $160 \mathrm{MeV}$, increase the beam intensity by about two orders of magnitude, and to provide the capability of fast reversal of the beam helicity with precision control and diagnostics of the spatial and energy distributions of the beam. These updates will enable the planned program in low-energy QCD, which includes measurements of nucleon structure using Compton scattering, photodisintegration of few-nucleon systems, investigations of charge symmetry breaking via $\pi^{0}$ production, and determinations of weak hadronic interactions using parity-violating photodisintegration of the deuteron. A two-stage upgrade is envisaged. The first, starting in 2018, is an upgrade of the electron injector system to increase the average charge injection rate into the storage ring and to enhance the overall reliability of the accelerator drivers. This upgrade will provide the $\gamma$-ray beam capabilities needed to carry out the planned Comptonscattering research program and the accelerator features needed for hadronic parity-violation experiments. The estimated cost is $\$ 2.5 \mathrm{M}$ to $\$ 3.5 \mathrm{M}$, depending on whether new FEL wigglers are required to meet the optical photon requirements for producing $\sim 120 \mathrm{MeV} \gamma$-ray beams with sufficient intensity to perform nuclear-physics research. The second stage is the installation of an optical cavity in the straight section of the storage ring that is driven by a high-powered external laser. The photons in the optical cavity will produce $\gamma$-rays by Compton backscattering from the electron bunches circulating in the storage ring. This source configuration is referred to as HI $\gamma \mathrm{S} 2$ and would start construction around 
2021; the estimated cost is $\$ 2 \mathrm{M}$. The $\gamma$-ray flux at HI $\gamma \mathrm{S} 2$ will be about $10^{11}$ to $10^{12} \gamma / \mathrm{s}$ in the energy range of 2 to $12 \mathrm{MeV}$, which will be comparable to the expected flux of the ELI-NP faculty in BucharestMagurele. In addition to the accelerator upgrades, target-room equipment must be developed, e.g., a scintillating polarized hydrogen target for nucleon spin polarizability measurements, $\gamma$-ray detectors for the $\pi^{0}$ measurements, and a liquid deuterium target and a nearly $4 \pi$ high efficiency neutron detector system for parity-violation measurements. The estimated cost of the target-room equipment is $\$ 1.0 \mathrm{M}$ for the nucleon spin-polarizability and $\pi^{0}$ production measurements and $\$ 0.6 \mathrm{M}$ for the parity-violation measurements on the deuteron.

\section{HRS - The High Rigidity Spectrometer}

The High Rigidity Spectrometer (HRS) facilitates experiments with the most neutron-rich nuclei at the highest rates available from FRIB. To accomplish this, the maximum magnetic rigidity of the HRS (8 $\mathrm{Tm}$ ) will match the rigidities for which rare-isotope production yields at the FRIB fragment separator are at the maximum across the entire chart of nuclei. The HRS will consist of a sweeper stage that separates charged fragments from reactions with fast beams from neutrons, and a spectrometer stage required to acquire the necessary momentum and particle-identification resolution. It will be fed by a largeacceptance beam-line that minimizes transmission losses from the FRIB fragment separator to the HRS. The program afforded by the HRS allows the scientific community to efficiently address important questions related to all four scientific challenges posed in the NRC decadal study "Nuclear physics: Exploring the Heart of Matter" and covers 13 of 17 benchmark programs introduced by the 2007 NSAC RIB Task Force. At the same time, the HRS extends the scientific reach and discovery potential of FRIB by enabling a diverse set of experiments up to the highest rare-isotope beam energies $(\sim 200 \mathrm{MeV} / \mathrm{u})$ available and maximizes the scientific output from other state-of-the-art and community-priority devices, such as the Gamma-Ray Energy Tracking Array (GRETA) and the Modular Neutron Array (MoNALISA), in addition to enabling diverse programs pursued with a multitude of ancillary detectors. Details about the scientific program (motivated by scientists from over 20 institutions) and the pre-conceptual design of the HRS can be found in the HRS whitepaper.

\section{HRTPC-AT - High Rigidity Time Projection Chamber - Active Target}

One of the needs for new FRIB equipment is for a highly-segmented time projection chamber for the measurement of in-flight charge particle decays (of unbound states of exotic rare isotopes) and for a program to investigate the equation-of-state (EOS) of neutron matter. The device would be similar to the S $\pi$ RIT Detector built for RIKEN. The EOS program will use high-energy ions to compress nuclear matter and provide information on matter $2 \mathrm{x}$ to one-tenth normal nuclear matter. For example, pion production is particularly sensitive to the assumed EOS and detection of charged pions can be used to test models. The primary goal is to increase the sensitivity of observables to the symmetry energy using rare isotope beams with extreme N/Z numbers and to assess and reduce the model dependence by requiring consistency with a "large" range of "reliable" observables. Highest sensitivity to symmetry energy will be obtained with the most neutron-rich systems. Another aspect of the program will be in conjunction with the proposed High Rigidity Spectrograph (described above) for coincidence studies where the TPC will act as an active target and light charged particle recoils can be measured in coincidence with heavy fragments. An example is giant resonance studies, e.g. the giant monopole resonance, using (d,d') on beams of doubly magic ${ }^{132} \mathrm{Sn}$ or exotic isotopes such as ${ }^{70} \mathrm{Ni}$.

\section{ISLA - The Recoil Separator for ReA12}

The FRIB and NSCL user communities have strongly endorsed the early implementation of reaccelerated beams with energy up to $12 \mathrm{MeV} / \mathrm{u}$ at FRIB. This capability would provide a world-unique set of beams from in-flight separation and gas stopping at energies compatible with many of the experimental and theoretical tools necessary for the FRIB scientific program. A recoil spectrometer coupled to ReA12 is needed to address large parts of the nuclear physics mission of this program-for aspects from nuclear 
structure and nuclear astrophysics to applications of rare isotopes-including the four overarching questions in the recent NRC decadal study of nuclear physics and a majority of the Benchmarks from the 2007 NSAC RIB Taskforce report. In the energy domain of ReA12 rare isotopes will be produced with many charge states after a reaction. The unambiguous identification of nuclei in this mass region and at low to medium energy will be a necessary and challenging task. Only a spectrometer specifically designed for this task can meet these needs. Besides excellent particle identification, the spectrometer is needed to reject unreacted beam. In many experiments it will be used in coincidence with detector arrays around the target, to clearly associate a particle or gamma detected at the target with a given reaction product.

A single spectrometer with these characteristics has emerged from the discussions of the community. The ISLA device has the above characteristics and will be world unique with its high M/Q resolving power of up to 10000 due to its isochronous properties. Its cost has been estimated to be about $\$ 10.0 \mathrm{M}$ with a $20 \%$ contingency included. This spectrometer would ideally be made available prior to FRIB start-up, in 2020 to 2022, to make the most of early research opportunities and to develop expertise to operate the spectrometer and its detectors efficiently.

\section{JENSA}

JENSA is a gas jet designed to produce targets with areal densities of $10^{18}-10^{19}$ atoms $/ \mathrm{cm}^{2}$ of hydrogen or helium. It was commissioned at ORNL and installed at the ReA3 facility of NSCL and will be used for light ion scattering, transfer, and capture experiments. In the future, it will be paired with the FRIB recoil separator SECAR to study capture reactions of astrophysical interest. The large target chamber can accommodate a myriad of detectors systems, such as high-segmentation silicon for charged particles (ORRUBA, superORRUBA, etc), scintillator arrays for $\gamma$-ray detection, and/or an ionization chamber on the beam axis to measure beam purity and heavy reaction products for low (sub pA) intensity beams. Planned upgrades include a cryogenic gas chiller $(\sim 40 \mathrm{k})$ and hermetically-sealed, higher throughput pumps ( \$200k), both of which will allow for increased target densities (roughly x2) and cleaner ${ }^{3} \mathrm{He}$ operations.

\section{Multi-user Capability at FRIB}

The importance of optimizing the availability of radioactive isotopes for research at FRIB cannot be overstated given the expense and difficulty in producing exotic isotopes. Therefore, secondary or parasitic systems are being developed for NSCL to catch and use isotopes that otherwise would be lost at various points in the primary fragment separator. These systems could then be modified and modernized for deployment at FRIB. One system currently being installed at NSCL is a He-jet-aerosol transport capillary that will deliver radioactive atoms to an ion source on the ReA3 accelerator. This system is often used to produce beams of refractory elements such as Zr-Pd. A hot graphite catcher will be developed to be placed in front of the slit system of the A1900 and will provide much higher intensities of most volatile elements. It is envisioned that this system would feed an ion source and mass analysis system to provide slow $(<300 \mathrm{keV})$ beams of exotic isotopes for research such as decay spectroscopy. These beams could, in principle, be more intense than those of the primary user since the ion source and mass analysis provides isotope identification allowing the use of non-fully stripped ions. Thus, the decay of heavy isotopes $(A>150)$ could be more easily studied at NSCL. Total cost depends on the sophistication of the final installation. A simple ion source and mass analysis system can cost less than $\$ 1 \mathrm{M}$ but an isobar separator and laser ionization could increase this by a factor of 2 or 3 .

\section{The N=126 Factory}

Isotopes in the very heavy neutron-rich region east of $\mathrm{N}=126$ are critical for our understanding of the heaviest abundance peak in the r-process. This region is however not readily accessible by standard fusion, fragmentation or fission reaction mechanisms. Deep-inelastic reactions can populate this region but the kinematics of the reaction recoils is such that it has been difficult to isolate and collect these 
nuclei. The $\mathrm{N}=126$ factory proposed at ANL uses the unique opportunity provided by the very intense heavy-ion beams available at ATLAS with the gas catcher technology developed at ANL for CARIBU and FRIB to make these isotopes available to the community for high-precision mass, lifetime and decay measurements. This new venture will allow a first exploration of this critical region and provide first level information to confirm assumptions about the nuclear structure in this region and how it affects rprocess abundances. This new facility, when coupled to the multi-user upgrade of ATLAS, would also enable the post-acceleration of these rare nuclei and allow investigation of other properties critical to our understanding of fission recycling in r-process nucleosynthesis.

\section{Solenoid Spectrometers}

One approach to reactions in inverse kinematics that has been shown to possess distinct advantages over more conventional techniques is to use a large-bore superconducting solenoid magnet as a particle spectrometer. Such a device can study reactions with reaccelerated beams with a simplified kinematic response that is similar for all reactions, and can provide very good resolution of excited states in the residual nucleus. An example of this approach has been in operation since 2008 with the HELIOS (HELIcal Orbit Spectrometer) at the ATLAS facility at Argonne National Laboratory. There, HELIOS has already been used to measure $(\mathrm{d}, \mathrm{p}),\left(\mathrm{d},{ }^{3,4} \mathrm{He}\right),\left({ }^{3} \mathrm{He}, \mathrm{d}\right),\left({ }^{6} \mathrm{Li}, \mathrm{d}\right)$ and $(\mathrm{t}, \mathrm{p})$ reactions with a variety of stable and unstable beams with $\mathrm{A}=11$ to 136 . Such a device can play an important role at future reaccelerated-beam facilities, in particular FRIB. As the technology has already been demonstrated to work, no additional research and development effort is necessary and a spectrometer similar to HELIOS could be ready for operation on or even before FRIB day-1 operations. Different options are being considered for such an implementation, such as sharing a large magnet with other instruments, and a possible coupling of a solenoid spectrometer with a recoil separator for experiments with heavy beams in very inverse kinematics. 


\subsection{Applications and Nuclear Data}

The impact of the research described in this session is ubiquitous, solves a wide range of "real world" problems, and dramatically improves people's lives. This research is diverse and hard to cover completely in a short section. Applied nuclear physics research is important for national security, medicine, nuclear energy, industry, materials, and education and the nuclear data derived from such research needs to be accurate, reliable and accessible to many different research communities.

\subsubsection{Accomplishments Since the Last Long Range Plan}

Because the topic area is so broad and diverse, it is difficult to fully enumerate the many accomplishments since the last long-range plan in all areas of applied nuclear physics. Certainly the development of new experimental techniques, more comprehensive theories, and reliable nuclear data, including the everexpanding number of known nuclides, has not only benefited applied research but the basic research community as well. During this time period, there has been an increasing recognition of the important contributions of applied nuclear research to nuclear physics in general. Some recent highlighted accomplishments in national security, medicine, nuclear energy, industry and materials, education and the nuclear data program are:

- National Security - Increased national security has resulted from improved understanding of fission dynamics through fission product yield measurements with mono-energetic neutrons, cross-section measurements on stable and unstable nuclei, and utilization of novel radiation detectors to protect borders and monitor worldwide nuclear activities. Also, measurements of gamma-ray induced reactions, e.g., nuclear fluorescence resonance, photo-neutron production and photofission, have contributed to the database used for developing new technologies for remote analysis of nuclear processes and forensic investigations.

- Medicine - Development of increased production of ${ }^{82} \mathrm{Sr} /{ }^{82} \mathrm{Rb}$ as medical isotopes important for cardiac positron emission tomography has progressed to the point where commercial production of the isotope is possible.

- Nuclear energy - Development of new techniques to determine beta-decay properties and reaction cross sections more precisely which improve nuclear reactor safety calculations.

- Industry and Materials - Improved understanding of single-event upsets in electronics with the utilization of energetic neutrons that more realistically describe cosmic ray generated neutrons at altitudes important for the aviation and space industries.

- Education - Nuclear chemistry and nuclear physics summer schools and the ubiquitous involvement of students and post-doctoral researchers in applied nuclear physics research has contributed significantly to the training of the next generation of scientists and engineers.

- Nuclear Data - The nuclear data program in the U.S. has dramatically improved the accuracy, reliability, and accessibility of nuclear data available for the scientific community.

\subsubsection{Applications}

\subsubsection{National Security}

The Stockpile Stewardship Program (SSP) is responsible for ensuring the safety and reliability of the nation's nuclear weapons without resorting to testing. This requires improving the quality and availability 
of nuclear data used for interpretation of nuclear events. This is accomplished with a combination of robust experimental efforts at current and future facilities, such as FRIB, and theory. Isotopes of interest span a range of half-lives which means a combination of experimental techniques will be required to make new measurements. Nuclear data improvements needed include better measurements of half-lives, decay branching ratios, reaction cross-sections, level density and $\gamma$-ray strength functions. Two examples illustrate some of the needs: 1) any measurements on reaction cross-sections induced on short-lived fission products which require in-beam experiments, and 2) measurements on reaction cross-sections of radioactive nuclei for which isotope harvesting is beneficial.

Improved knowledge of production and destruction reactions for $\mathrm{A}=95$ fission products is desired. The cross-sections of interest within the region are shown in Fig. 1, with ${ }^{95} \mathrm{Sr}$ being perhaps the most important nuclide to obtain experimentally determined cross-sections. Generation of ${ }^{95} \mathrm{Sr}$ beams at CARIBU or FRIB, coupled with developments of inverse kinematics reactions such as (d,p) reactions, will enable experimental determination of a few key cross-sections and improve the theoretical models used to calculate the other reactions and cross-sections. Experimental data for several other mass regions, $\mathrm{A}=144$ and $\mathrm{A}=147$, is desired.

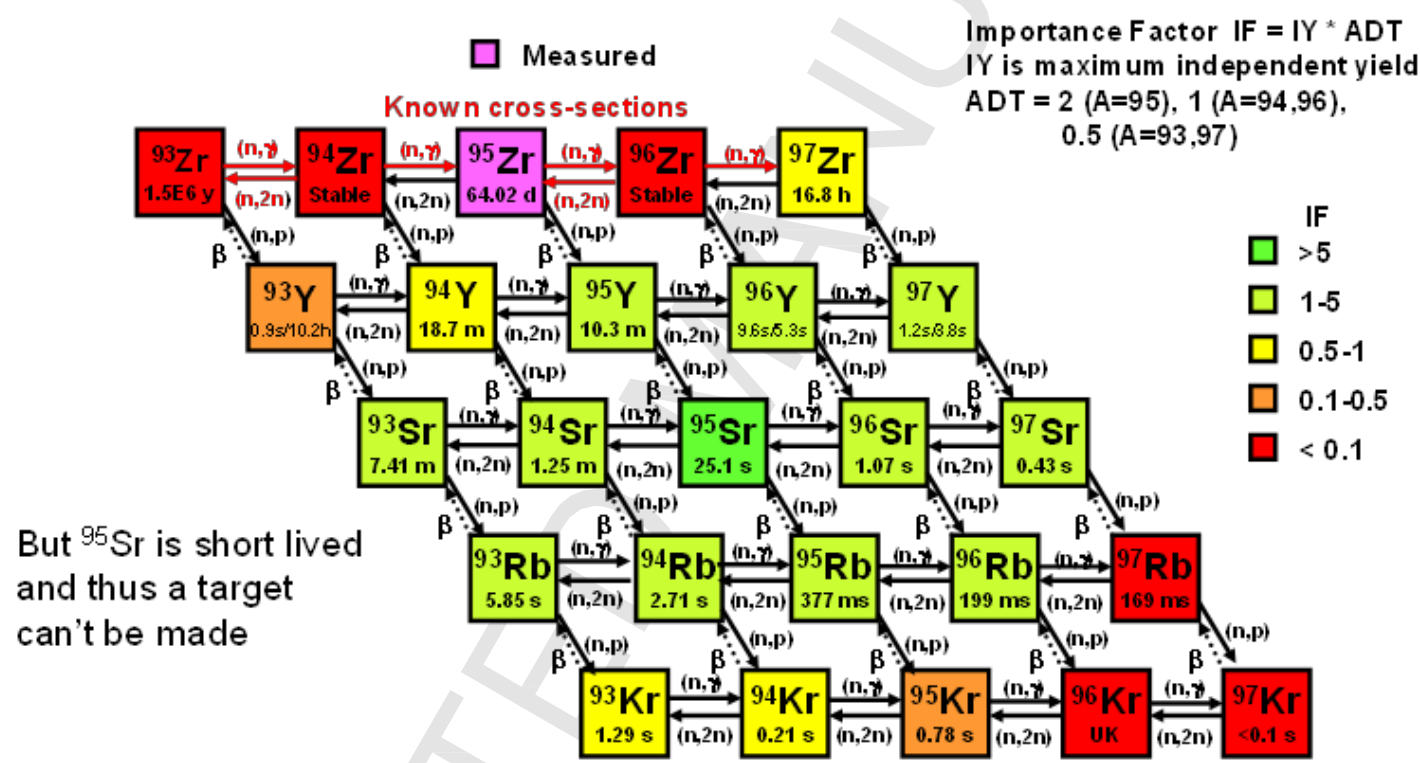

Figure 1: Part of the chart of nuclides showing the neutron rich fission products of interest to the SSP.

Isotopes such as ${ }^{48} \mathrm{~V}$ and ${ }^{88} \mathrm{Zr}$ are suitable for isotope harvesting and construction of targets for conventional neutron induced experiments. In many such cases, experimental cross-section information is completely lacking, such as for ${ }^{88} \mathrm{Zr}(\mathrm{n}, \gamma)^{89} \mathrm{Zr}$. This effort is synergistic with the medical community's development of ${ }^{89} \mathrm{Zr}$ as a medical imaging diagnostic isotope. Isotope harvesting schemes to obtain ${ }^{67} \mathrm{Cu}$ from the FRIB beam dump water have shown significant promise-additional chemistry to purify $\mathrm{Zr}$ isotopes is under development.

Improved knowledge of cross-sections is also important in the field of inertial confinement fusion both to diagnose the performance of the experiments and to investigate the influence of high energy density plasmas on nuclear data such as reaction cross-sections at elevated temperatures and isomeric state populations. Examples include improved measurements of the $T(D, \gamma) / T(D, n)$ branching ratio for interpretation of the gamma reaction history diagnostic used at the National Ignition Facility (NIF) and other laser facilities. In addition, threshold nuclear reactions such as ${ }^{12} \mathrm{C}(\mathrm{n}, 2 \mathrm{n}){ }^{11} \mathrm{C}$ or ${ }^{169} \mathrm{Tm}(\mathrm{n}, 3 \mathrm{n}){ }^{167} \mathrm{Tm}$ can be used to diagnose high energy $\left(E_{n}>14 \mathrm{MeV}\right)$ neutrons produced during ICF implosions. Finally, radiochemical diagnostics (charged particle and neutron induced reactions) can be used to determine if 
mix of unwanted material into the fusion fuel occurs and to what extent such mix affects the capsule performance.

\subsubsection{Medical Research}

Nuclear medicine uses radioactive material for safe and cost-effective imaging and treatment of illnesses. Modern technologies allow radioisotopes to be delivered directly to their targets - diseased cells. They emit radiation that allows specialists to image the extent of a disease process in the body, based on cellular function and physiology, allowing doctors a better understanding of the diseased tissue than other diagnostic procedures, which may only capture structural information. New radiopharmaceuticals for these imaging techniques can be developed to help determine which patients may respond to which treatment, paving the way for personalized medicine. Radionuclides are generally used to 'label' a radiopharmaceutical. The overall chemical structure of the radiopharmaceutical determines its biological properties, while the radionuclide determines the imaging or therapeutic properties. Investments in research and training for nuclear physics and chemistry can impact this important field in a variety of ways ranging from development of accelerator targetry to produce novel radioisotopes, to the development of new detectors for imaging techniques.

There are three major types of nuclear medicine imaging approaches: planar scintigraphy; single photon emission computed tomography (SPECT); and positron emission tomography (PET). Due to its high sensitivity and resolution, PET is fast growing as an imaging technology. The widespread use of this technology is based on the creation of specific imaging probes. For example, $\left[{ }^{18} \mathrm{~F}\right]$ Fluorodeoxyglucose $\left(\left[{ }^{18} \mathrm{~F}\right] \mathrm{FDG}\right)$ is an FDA approved drug used for imaging in many types of cancer for staging and assessment of response to therapy. Research in the PET imaging agent development area has been typically focused on 4 main radioisotopes (carbon-11, nitrogen-13, oxygen-15 and fluorine-18). However, their short half-lives restrict radiopharmaceutical development for those that examine fast biological processes. Research into new isotopes has, therefore, gathered much momentum in recent years.

The new isotopes allow for the innovative design and integration of a broader range of PET tracers to investigate biological activity and processes. Several targeting pharmaceutical agents slowly congregate at the site of a tumor, meaning longer-lived isotopes are often chosen to optimize the accumulation of target tissue, as well as abate the background uptake. More recent studies have focused on the PET radiometals: copper-64 $\left({ }^{64} \mathrm{Cu}\right)$, yttrium-86 $\left.{ }^{86} \mathrm{Y}\right)$ and zirconium-89 $\left({ }^{89} \mathrm{Zr}\right)$ with promising results. The employment of these non-traditional radionuclides is restricted by their availability as they are only produced at a few sites.

Additionally, new agents are being used for targeted radiotherapy. Recently, the FDA approval of ${ }^{223} \mathrm{Ra}$ for treatment of metastatic prostate cancer has sparked a resurgence of interest in the development of targeted radiotherapeutics. Alpha emitting isotopes such as ${ }^{225} \mathrm{Ac}$ and ${ }^{211} \mathrm{At}$ are promising for radiolabeling of the next generation of these compounds which deliver focused radiation at the diseased site, thus sparing normal tissues. Research and infrastructure to enable the widespread availability of these isotopes is vital for the clinical translation of these agents.

The development of new isotopes is a vital component of molecular imaging and therapeutic probe development. Chemical flexibility, coupled with a variety of half-lives, drive innovative development of novel agents, allowing the imaging of numerous biological processes. These studies are proving effective in the area of imaging and therapy of multiple disease processes. 


\subsubsection{Nuclear Energy}

The $\beta$-decay properties of fission products have an impact on a variety of applications of nuclear science involving fission such as nuclear energy production, nuclear safeguards, and stockpile stewardship. Although hundreds of radioactive isotopes are produced in fission, often detailed nuclear data on the $\beta$ decay properties of only a handful of key isotopes is needed. With high-quality fission-product beams now available at many radioactive beam facilities around the world, detailed studies of $\beta$-decay can be performed to improve the nuclear data. Below, the desired $\beta$-decay properties ( $\beta$-particle energy distributions, $\beta$-delayed $\gamma$-ray branching ratios, and $\beta$-delayed neutron yields and energy spectra) are described and certain key isotopes are identified.

\subsection{Beta Spectral Shapes}

Nuclear reactors provide the highest intensity source of "man-made" antineutrinos, which are emitted in the $\beta$ decay of all the neutron-rich fission products. As a result, these antineutrinos $\left(\bar{v}_{\mathrm{e}}\right)$ are of great interest for fundamental neutrino physics, nonproliferation monitoring, and reactor physics. The need to precisely understand the $\bar{v}_{\mathrm{e}}$ spectra emitted from the products of fissioning systems is underscored by the first measurements of $\theta_{13}$ which suggested that there may be a reactor antineutrino anomaly caused by sterile neutrinos.

Much effort has focused recently on the reactor antineutrino anomaly where the measured $\bar{v}_{\mathrm{e}}$ flux is in disagreement with what has been inferred from calculations of the $\bar{v}_{\mathrm{e}}$ spectra, which combine information from nuclear databases with measured total electron spectra from the major actinides. Combining data on 18 short-baseline reactor antineutrino experiments yields a ratio of observed to predicted event rate of $0.943 \pm 0.023$, a $2.5 \sigma$ difference from unity. However, the use of this neutrino signal for applications and for fundamental neutrino physics is greatly strengthened by a sound basis of the underlying $\beta$-decay spectra from fission fragments.

The $\bar{v}_{\mathrm{e}}$ spectrum can be calculated by summing the individual $\beta$-spectra for each fission fragment weighted by its cumulative fission yield. Although there are hundreds of radioactive isotopes produced in fission, only a handful of nuclei have significant influence on the spectrum. For example, the nuclei ${ }^{92} \mathrm{Rb}$ and ${ }^{96} \mathrm{Y}$ are the first and second largest contributors to the neutrino spectrum, each making up $6 \%$ at 4 $\mathrm{MeV}$ and $21 \%\left({ }^{92} \mathrm{Rb}\right)$ and $15 \%\left({ }^{96} \mathrm{Y}\right)$ at $5.5 \mathrm{MeV}$ of the total $\bar{v}_{\mathrm{e}}$ spectrum. Given the significance of the $\beta$ energy spectra of a handful of key fission products to the $\bar{v}_{\mathrm{e}}$ spectrum calculations, these isotopes should be studied using $\beta$-spectroscopy and discrete $\beta$-ray spectroscopy.

\subsection{Beta-delayed $\gamma$-ray Branching Ratios}

The fission products ${ }^{147} \mathrm{Nd},{ }^{144} \mathrm{Ce},{ }^{156} \mathrm{Eu}$, and certain other long-lived isotopes play a crucial role in sciencebased stockpile stewardship because determining the yield of these isotopes is one of the most straightforward and reliable ways to determine the number of fissions that occurred in a nuclear weapon test. The yield of these fission products is most precisely determined by detecting the characteristic $\gamma$-rays emitted during the $\beta$ decay of these long-lived fission products. These $\gamma$ rays are emitted in only a fraction of the decays, and this fraction (the $\gamma$-ray branching ratio) must be known accurately to determine the total number of fissions. However, large nuclear-decay uncertainties are currently limiting the usefulness of the existing data. The absolute $\gamma$-ray branching ratios for ${ }^{147} \mathrm{Nd},{ }^{156} \mathrm{Eu}$, and ${ }^{144} \mathrm{Ce}$ are desired to higher precision for stockpile-stewardship applications and to greatly improve the precision and reliability with which the number of fissions can be determined. 
In addition, fission-product yields are typically determined using $\gamma$-ray spectroscopy and in these cases the $\gamma$-ray branching ratios are often the largest source of uncertainty in the determination of fissionproduct yields. For example, recent measurements at the Triangle Universities Nuclear Laboratory (TUNL) exploring the neutron-energy dependence of fission product yields would benefit from an improved absolute normalization that could be provided by branching ratio measurements. Improved fission-product distributions would also benefit a variety of applications such as nuclear reactor design and $\gamma$-ray based safeguard applications as well as to guide fundamental theories of fission.

The central challenge in measuring these $\gamma$-ray branching ratios is to determine the total number of decays of the nucleus of interest. Properly accounting for sample limitations (impurities, self-attenuation, etc.) and any accompanying conversion electron emission play an essential role in determining the overall normalization for the decay. These challenges can be largely circumvented by producing ultra-thin samples nearly free of radioactive contaminants at the CARIBU Facility at Argonne National Laboratory and measuring the absolute number of decays utilizing a $4 \pi \beta$ spectrometer, which can be essentially $100 \%$ efficient for a well-designed instrument, in conjunction with precision $\gamma$-ray spectroscopy.

\subsection{Beta-delayed Neutron Emission}

The neutrons emitted following the $\beta$ decay of fission products (known as delayed neutrons because they are emitted after fission on a timescale of the $\beta$-decay half-lives) play a crucial role in reactor performance and control. Reviews of delayed-neutron properties highlight the need to obtain high-quality data for a wide variety of delayed-neutron emitters to better understand the time-dependence and energy spectrum of the neutrons as these properties are essential for a detailed understanding of reactor kinetics needed for reactor safety and to understand the behavior of these reactors under various accident and component-failure scenarios. For fast breeder reactors, criticality calculations require accurate delayedneutron energy spectra. Approximations that are acceptable for light-water reactors such as assuming the delayed-neutron and fission-neutron energy spectra are identical are not acceptable, Boltzmann Uehling Uhlenbeck and improved $\beta$-delayed neutron data is needed for safety and accident analyses for these reactors. With improved nuclear data, the delayed-neutrons flux and energy spectrum could be calculated from the contributions from individual isotopes and therefore could be accurately modeled for any fuelcycle concept, actinide mix, or irradiation history.

However, spectroscopy of $0.1-$ to $10-\mathrm{MeV}$ neutrons is challenging and the quality of the data available today for individual nuclei is limited - in some cases discrepancies as large as factors of 2-4 in recent measurements of $\beta$-delayed neutron branching ratios have been uncovered and for the vast majority of neutron emitters, the energy spectrum has not been measured. Due to the challenges and limitations associated with existing techniques, a new approach to performing $\beta$-delayed neutron spectroscopy would be valuable for collecting the high-quality, reliable data needed for applications.

Ion traps can have as large an impact on the study of $\beta$-delayed neutron emission as they have had in the field of mass spectrometry. Trapped radioactive ions suspended in vacuum allow a new way to measure delayed-neutron properties by inferring the neutron energy from the large momentum kick it imparts. When a radioactive ion decays in the trap, the recoil-daughter nucleus emerges from the $\sim 1-\mathrm{mm}^{3}$ trap volume without scattering and the recoil energy can be measured. The energy of the emitted neutron can be easily and precisely reconstructed from this recoil by conservation of energy/momentum. Because of the massiveness of the neutron relative to the emitted leptons, the recoil energy is dominated by the neutron recoil. This ability to measure the nuclear recoil unperturbed enables a novel way to perform delayed-neutron spectroscopy with high efficiency, few backgrounds, and improved energy resolution. All the well-known challenges associated with detecting neutrons are avoided. The delayed-neutron 
branching ratios and neutron energies can be precisely determined by detecting the recoil ions in coincidence with the $\beta$ particles.

The first-ever demonstration that $\beta$-delayed neutron measurements could be made by studying the nuclear recoil was recently performed using the beta-decay Paul Trap instrumented with $\beta, \gamma$-ray and recoil-ion detectors (see Fig. 2). The $\beta$-delayed properties of ${ }^{137}$ I were studied and the measured neutron branching ratio and energy spectrum were consistent with previous direct measurements, paving the way for future studies of beta-delayed neutron emission.

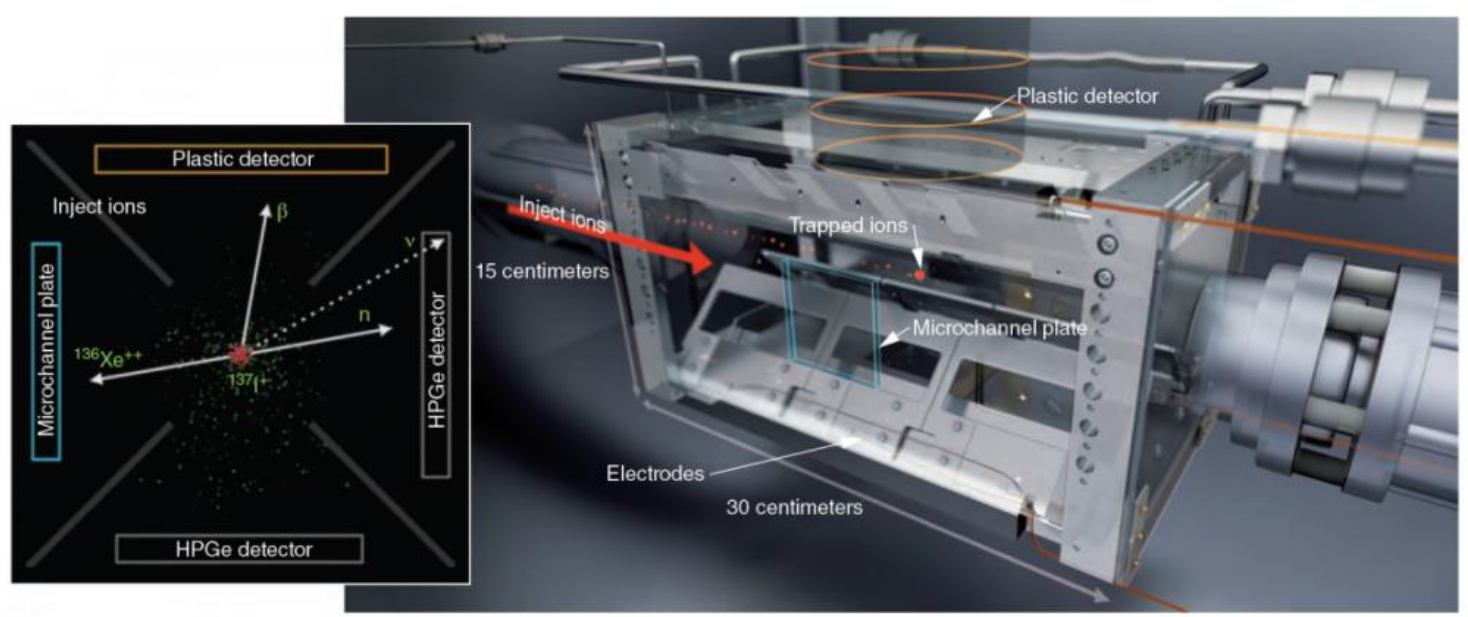

Figure 2: An illustration of the beta decay Paul Trap and the recoil-ion approach to $\beta$-delayed neutron spectroscopy. The box on the left shows a cross-sectional view of the ion cloud, electrode, and detector array geometry. Neutron emission is inferred from the time-of-flight of the recoil ion detected in coincidence with the emitted $\beta$ particle. The graphic is from the September 2013 issue of LLNL's Science and Technology Review magazine.

\subsubsection{Industry and Materials}

Nuclear physics applications in industry and materials encompass a wide variety of uses from nondestructive assay and analysis of manufactured items, accelerated wear testing of critical components in moving assemblies, determination of flow pathways and dynamics in complex systems including the environment, and protection of electronic components from single event upsets due to cosmic rays. Many of these applications rely on nuclear physics expertise and/or experimental capabilities.

Accelerated testing of electronic components occurs at several accelerator facilities, with industrial companies interested in matching cosmic ray fluxes and intensities with cocktails of charged particle beams or neutrons with the appropriate energy spectrum. One of the most concerning types of single event effects observed in electronics is due to cosmic ray induced neutrons because of the long mean-free paths of neutrons in the atmosphere and materials. This is of extreme concern to the aviation and aeronautics industry because aircraft fly at altitudes where fluxes of neutrons are over two orders of magnitude higher than at ground level. The neutrons are produced by cosmic rays in the upper atmosphere, penetrate to lower altitudes, interact with $\mathrm{Si}$ atoms in the computer/electronics components creating charged particles that recoil through the material, and deposit charge in sensitive volumes of the material causing the state of a node to change resulting in a variety of effects that are observable in the device like data corruption or single event latch-ups, burn-ups, or gate ruptures. Industry trends to lower 
An avionics incident highlights the single event effect problem in electronics equipment affecting people's lives. On October 7, 2008, Quantas 72 was en route from Singapore to Perth, Australia. Excerpts from the investigation report ATSB Transport Safety Report Aviation Occurrence Investigation AO-2008-70 indicate the seriousness of the issue. "While at 37,000 ft., one of the aircraft's three Air Data Inertial Reference Units (ADIRU) started outputting intermittent, incorrect values...Two minutes later ...the aircraft flight control primary computers commanded the aircraft to pitch down. At least 110 of the 303 passengers and nine of the 12 crew members were injured: 12 of the occupants were seriously injured and another 39 received hospital medical treatment."

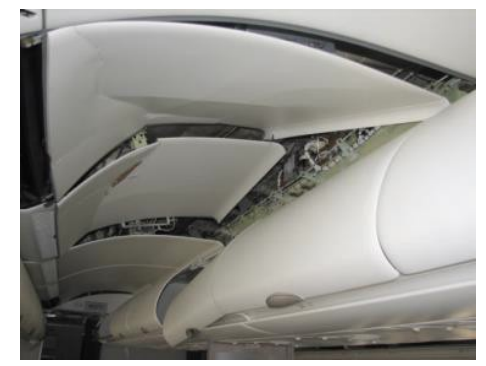

The report concluded that one possible cause was: "The other potential triggering event was a single event effect (SEE) resulting from a highenergy atmospheric particle striking one of the integrated circuits within the CPU module. There was insufficient evidence available to determine if an SEE was involved, but the investigation identified SEE as an ongoing risk for airborne equipment." Testing of components in this plane had occurred with $14 \mathrm{MeV}$ neutrons only, missing the large amount of more energetic neutrons present in cosmic ray induced neutrons. voltages and smaller feature size are thought to increase the failure rate due to these kinds of events. Similar devices can have very different failure rates, however, the failure rates due to these kind of single event effects are equal to all other kinds of failures in the devices combined.

Neutron experiments can be performed at Los Alamos National Laboratory at the LANSCE facility, where the neutron spectrum from the spallation neutron source has been measured to match the cosmic ray neutron spectrum. Accelerated testing of components can occur by operating devices while they are

being irradiated with neutrons and observing the single effect upset rate in either a component or whole device. Typically these irradiations occur over the course of a day or week of testing in order to develop equivalent single event rates due to cosmic ray neutrons. Cosmic ray neutrons affecting airplane computer systems [ATSB Transport Safety Report Aviation Occurrence Investigation AO-2008-70] and supercomputer calculations [IEEE Trans. Dev. Mat. Reliab. ㄷ2005] have been documented.

\subsubsection{Education}

Pervasive throughout all of the presentations and the discussion mentioned in this document is the important educational development of undergraduate and graduate students involved in all areas of research discussed. The importance of the students to the research, and the accessibility of the applied research and nuclear data for student research projects and theses, is not repeated in detail here because these important relationships have been stated more eloquently in numerous other forums and whitepapers; most recently in the whitepaper from the Education and Innovation town meeting held earlier at MSU. We underscore their recommendations and conclusions and note the contributions to nuclear data and applied nuclear research made in educating students.

\subsubsection{Nuclear Data: Accurate, Reliable and Accessible}

The primary activities of the U.S. Nuclear Data Program (USNDP) are to collect, evaluate, and disseminate nuclear physics data for basic nuclear physics and applied nuclear technology research. The 
nuclear data infrastructure provided by the USNDP impacts governmental, educational, commercial, and medical organizations in United States, and is part of the U.S. commitment to the international nuclear data networks. Presentations in this session described examples of nuclear data measurements carried out at ANL, LANL, LBNL and TUNL; these measurements have an impact on, for example, parity violation limits, astrophysics, medical isotope research, stockpile stewardship, and measurements of "enabling data" that are useful or necessary for analysis of data from other experiments. In each case, improvement in the accuracy and precision of the data can provide improvement in some associated underlying science or application. Throughout the session there was an unresolved discussion theme connected with associating the term "applications data" with basic science measurements that have a strong association with meeting the nation's nuclear data needs. In any case, it is evident that the need for convenient access to the best nuclear data is increasing, and therefore it is critically important to maintain a high level of expertise in the area of nuclear data evaluation to assure the continuation of the nuclear databases with sufficient breadth and quality to meet the requirements of advanced computational applications.

The domestic and global demand for nuclear data continues to increase, as nuclear science and technologies find new utility in our advancing society. Detailed and complex nuclear data and associated libraries are now commonplace in many applied fields, including energy, medicine and security among others. The collections of nuclear data libraries represent the collective annals of nuclear science research and discoveries. Insights to new discoveries benefit greatly from systematic analysis of prior studies, and from the ability to access these data promptly. Under these circumstances, the convenient availability of comprehensive, up-to-date, and well-ordered databases is an essential tool for the nuclear physics research community and applications specialists who need such data as part of their daily routine. Such credible and reliable libraries act as a bridge between science, technology, and society by making the results of basic nuclear physics research available to a broad audience of users, and hence, having a profound effect on the socioeconomic applications of modern nuclear science.

The basic and applied science research communities that fulfill these demands are expanding the knowledge pool, as well as improving the accuracy of the data, which can have significant impact on industrial applications. Worldwide efforts are coordinated primarily by the International Atomic Energy Agency in Vienna (IAEA) and the Nuclear Energy Agency of the Organization for Economic Cooperation and Development (NEA-OECD) in Paris. The development and maintenance of nuclear data libraries, and dissemination of nuclear data to various user communities is the main goal of the international networks associated with these agencies: the Nuclear Reaction Data Centres Network (NRDC/IAEA), the Nuclear Structure and Decay Data evaluators (NSDD/IAEA), and the Working Party on International Nuclear Data Evaluation Co-operation (WPEC/NEA). More information about them can be found at http://www-nds.iaea.org/nrdc.html, http://www-nds.iaea.org/nsdd, and http://www.nea.fr/html/science/wpec.

The DOE/SC-funded U.S. Nuclear Data Program (USNDP) comprises nuclear data experts from national laboratories and academia across the United States who collect, evaluate, and disseminate nuclear physics data for basic nuclear physics and applied nuclear technology research. The focal point of the network is the National Nuclear Data Center (NNDC) at Brookhaven National Laboratory, which is the major outlet for storing and disseminating nuclear data in the U.S. The services provided by the national network of nuclear data evaluation groups are essential to organizations with missions that require access to nuclear data. The nuclear data infrastructure provided by the USNDP impacts governmental, educational, commercial, and medical organizations in United States, as illustrated in Figure 3, and is part of the U.S. commitment to the international nuclear data networks. 


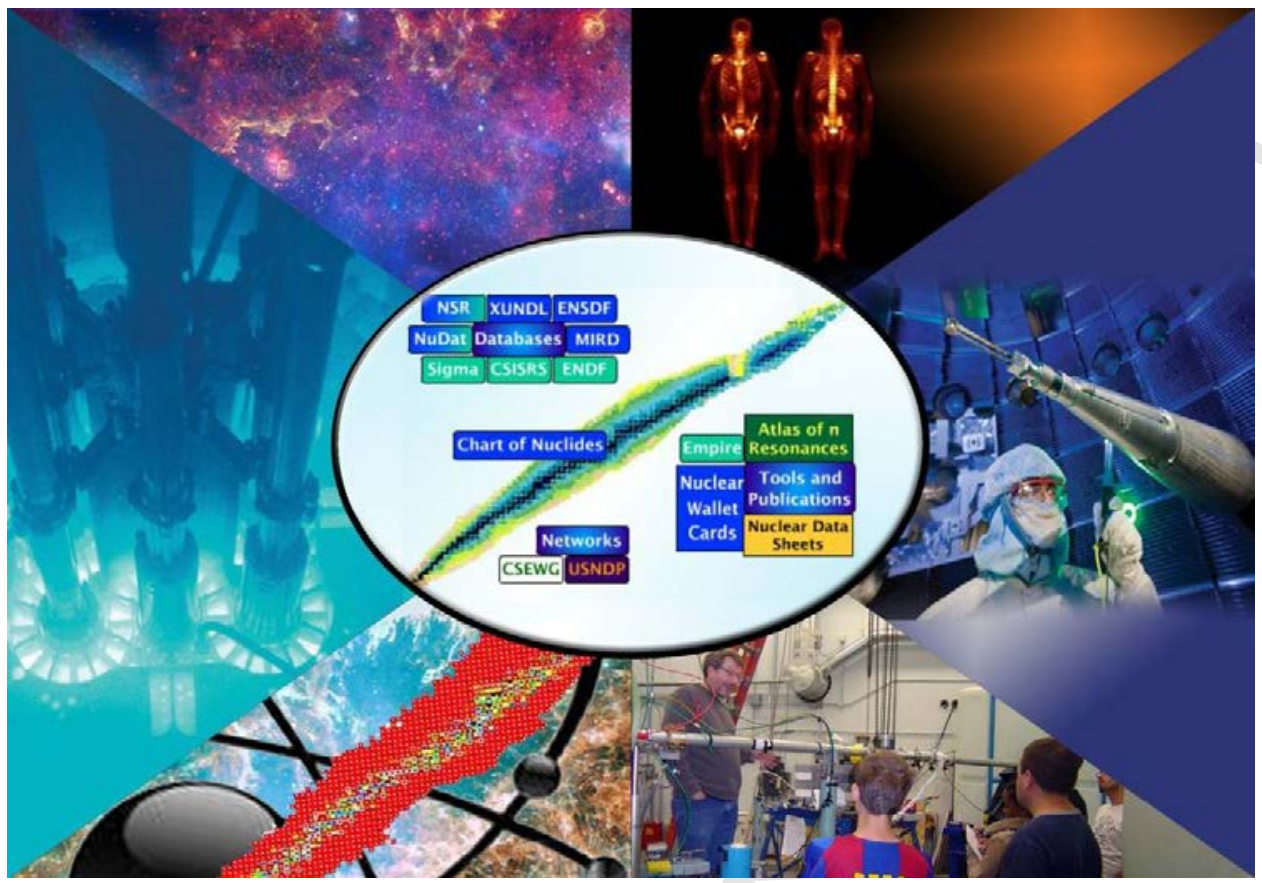

Figure 3: The core nuclear physics databases and products of the USNDP, and their main areas of impact for science and technology.

The mission of the USNDP is to provide current, accurate, authoritative data for workers in pure and applied areas of nuclear science and engineering. This is accomplished primarily through the compilation, evaluation, dissemination, and archiving of extensive nuclear datasets. The USNDP also addresses gaps in the data, through targeted experimental studies and the use of theoretical models. By carrying out this mission over the next decade, the USNDP network will continue to develop and maintain key components that motivate basic science discovery and support the progress of advanced simulation codes. The critical issue is to maintain a high level of expertise in the area of nuclear data evaluation to assure sufficient breadth and quality of the nuclear databases and to meet the requirements of a continuously developing user community.

\subsubsection{Common Themes}

Several common themes emerge from this diverse topic area. Applied research often benefits basic research (and vice versa) and a strong synergy exists between basic and applied research - there are many connections between the communities and the scientific work that are mutually beneficial. There is a diversity of funding sources and laboratories pursuing applied nuclear science, with much of the research resulting in significant contributions (PRLs, etc.) to nuclear science. Modest investment in nuclear data and technologies has large impact on nuclear science and education and opportunities exist for complementary nuclear data efforts. The economic and societal impact of this research is huge in areas of national security, medicine, nuclear energy, industry, materials, and education. Examples include: SingleEvent-Upset electronics testing that benefits the airline industry - affecting 825 million passengers last year; Nuclear power industry that generates $\sim 20 \%$ of US electricity - affecting 60 million US residents annually; ${ }^{99 m}$ Tc for medicine used in 20 million patient treatments last year; and NNDC nuclear data retrievals of more than 3 million last year. The impact of the research described in this section is ubiquitous, solves a wide range of “real world” problems, and improves people’s lives 


\subsubsection{Recommendations}

The Working Group developed one recommendation for each area as indicated below:

Applications

- Because of the broad economic and societal benefits of applied nuclear physics efforts to national security, medicine, nuclear energy, industry, materials, and education, we recommend increased support for innovation and applications within DOE/SC/NP and NSF.

Nuclear Data

- Because of the critical and pervasive use of nuclear data in a variety of basic and applied scientific efforts, we recommend strong support for the US Nuclear Data Program, which includes timely compilation, evaluation, secure archiving, and dissemination of nuclear data.

We recognize the similarities between the recommendation from the Education and Innovation town meeting held earlier at MSU and these, and therefore also endorse that particular recommendation. 


\title{
Appendix: Nuclear Astrophysics and Low-Energy Nuclear Physics Town Meetings Joint Program
}

\author{
Thursday, August 21 ${ }^{\text {st }}$ : Mitchell Physics Building \\ 8:15 AM - 10:35 AM Thursday Morning Joint Astro/LENP Plenary Session \\ Room: 203/204/205; Chair: M. Riley \\ 8:15 AM - 8:20 AM Welcome \\ 8:20 AM - 8:35 AM Charge and LRP process (J. Hardy) \\ 8:35 AM - 9:05 AM Theory of Nuclei and Their Reactions (W. Nazarewicz) \\ 9:05 AM - 9:35 AM Experimental Studies of Nuclei (R.V.F. Janssens) \\ 9:35 AM - 10:05 AM Nuclear Astrophysics Overview: Report from 2012 Town Meeting (H. \\ Schatz) \\ 10:05 AM - 10:35 AM Theory for Astrophysics (S. Reddy) \\ 10:35 AM - 11:00 AM Coffee Break
}

11:00 AM - 12:15 PM Thursday Morning Joint Astro/LENP Plenary Session

Room: 203/204/205; Chair: C. Elster

11:00 AM - 11:25 AM Fundamental Symmetries (B. Balantekin)

11:25 AM - 11:50 AM Neutrino Physics (N. Tolich)

11:50 AM - 12:15 PM Nuclear Structure Studies at Jefferson Laboratory (J. Arrington)

12:15 PM - 1:30 PM LUNCH

1:30 PM - 3:10 PM Thursday Afternoon Joint Astro/LENP Plenary Session

Room: 203/204/205; Chair: H. Schatz

1:30 PM - 1:50 PM FRIB Status, NSCL, and the Path Forward (T. Glasmacher)

1:50 PM - 2:10 PM FRIB Major Equipment (M. Smith)

2:10 PM - 2:30 PM ATLAS White Paper Summary (G. Savard)

2:30 PM - 2:50 PM ARUNA White Paper Summary (I. Wiedenhoever)

2:50 PM - 3:10 PM Experimental Initiatives in Nuclear Astrophysics (C. Brune)

3:10 PM - 3:35 PM Coffee Break

3:35 PM - 4:35 PM Thursday Afternoon Joint Astro/LENP Plenary Session Room: 203/204/205; Chair: M. Wiescher

3:35 PM - 3:55 PM Report from the Workshop on Computational Needs for Nuclear Physics (J. Carlson)

3:55 PM - 4:15 PM Report from the Workshop on Education and Outreach (M. Thoennesen) 4:15 PM - 4:35 PM Applications Overview (S. Wender)

Short break

4:45 PM - 5:45 PM Report from the Town Meeting on Education and Innovation Room: 203/204/205; Chair: (M. Thoennessen) 
6:00 PM - 7:30 PM Reception

Cyclotron Institute

Friday, August 22 ${ }^{\text {nd }}$ : Mitchell Institute / Mitchell Physics Building

8:30 AM - 12:00 PM LENP WG (Working Group Organizers in Parenthesis)

10:15 AM - 10:35 AM Coffee Break

- WG10: Theory for Low-Energy Nuclear Science (Carlson, Hagen, Elster, Reddy) Room 205

- WG11: Nuclear Structure and Reactions Experiment (Carpenter, Fallon, Gade, Higinbotham, Jones, Wuosmaa) Room Hawking Auditorium

- WG12: Nature of Dilute and Dense Nuclear Matter and the Equation of State (Horowitz, Natowitz, Sobotka, Tsang) Room 204

- WG14: Applications and Nuclear Data (Stoyer, Lapi, Vetter, Kondev, McCutchen) Room 203

12:00 PM - 1:00 PM Lunch

1:00 PM - 2:30 PM Astro WG 1 + LENP WG 2 (Working Group Organizers in Parenthesis)

- WG7: Supernova models, mergers models and chemical evolution (Hix, Froehlich) Room MI102

- WG2: RIB Beams, explosive nucleosynthesis (Rehm, Zegers, Bardayan, Blackmon) Room 204

- WG3: Stable and gamma beams, stars, .... (Champagne, Brune, Rogachev) Room 203

- WG1: Nuclear theory for astrophysics (McLaughlin, Reddy, Escher) Room 205

- WG14: Applications and Nuclear Data (Stoyer, Lapi, Vetter, Kondev, McCutchen) Room 213

- WG11: Nuclear Structure and Reactions Experiment (Carpenter, Fallon, Gade, Higinbotham, Jones, Wuosmaa) Room Hawking Auditorium

2:30 PM - 3:00 PM Coffee Break

3:00 PM - 4:30 PM Astro WG 2 (Working Group Organizers in Parenthesis)

- WG6: Astrophysics theory and computing (Timmes, Messer) Room 205

- WG4: Neutron beams, s-process, NIF (Couture, Bernstein) Room 213

- WG5: Data for Astrophysics (Meyer, Smith) Room 203

- WG8: Neutron Stars Science, experiments, theory (Lynch, Steiner) Room 204

4:30 PM - 4:45 PM Break

4:45 PM - 6:45 PM Joint Astro/LENP Session (Working Group Organizers in Parenthesis)

- WG13: Experimental Facilities and Instrumentation (Gross, Howell, Macchiavelli, Savard, Sherrill, Wiedenhoever) Room Hawking Auditorium

- Wg15: Future Computational Needs in Nuclear Physics (Carlson) Room 203

Saturday, August 23 ${ }^{\text {rd }}$, : Mitchell Physics Building

8:30 AM - 12:00 PM Joint Astro/LENP Session

Room: 203/204/205 
8:30 AM - 10:00 AM WG Reports

10:00 AM - 10:20 AM Coffee Break

10:20 AM - 12:00 PM Resolutions and Outcomes

12:00 Adjourn 